\title{
Modeling loss-propagation in the global supply network: The dynamic agent-based model acclimate
}

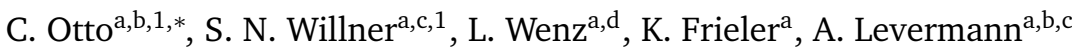 \\ ${ }^{a}$ Potsdam Institute for Climate Impact Research, Potsdam, Germany \\ ${ }^{b}$ Columbia University, New York, USA \\ ${ }^{c}$ University of Potsdam, Potsdam, Germany \\ ${ }^{d}$ Mercator Research Institute on Global Commons and Climate Change, Berlin, Germany
}

\begin{abstract}
World markets are highly interlinked and local economies extensively rely on global supply and value chains. Consequently, local production disruptions, for instance caused by extreme weather events, are likely to induce indirect losses along supply chains with potentially global repercussions. These complex loss dynamics represent a challenge for comprehensive disaster risk assessments. Here, we introduce the numerical agentbased model acclimate designed to analyze the cascading of economic losses in the global supply network. Using national sectors as agents, we apply the model to study the global propagation of losses induced by stylized disasters. We find that indirect losses can become comparable in size to direct ones, but can be efficiently mitigated by warehousing and idle capacities. Consequently, a comprehensive risk assessment cannot focus solely on first-tier suppliers, but has to take the whole supply chain into account. To render the supply network climate-proof, national adaptation policies have to be complemented by international adaptation efforts. In that regard, our model can be employed to assess reasonable leverage points and to identify dynamic bottlenecks inaccessible to static analyses.
\end{abstract}

Keywords: disaster impact analysis, higher-order effects, economic network, resilience, dynamic input-output model, agent-based modeling

JEL: F100, F180, Q540, Q560

\section{Please cite this paper as}

Otto C, Willner SN, Wenz L, Frieler K, Levermann A. (2017).

Modeling loss-propagation in the global supply network: The dynamic agent-based model acclimate.

Journal of Economic Dynamics and Control, 83, 232-269.

DOI: 10.1016/j.jedc.2017.08.001

\footnotetext{
${ }^{*}$ Corresponding author

Email address: christian.otto@pik-potsdam.de (C. Otto)

${ }^{1}$ with equal contributions
} 


\section{Introduction}

We here present the dynamic agent-based model acclimate describing the propagation of disaster-induced production losses in the global economic network. We define disasters as unanticipated local events leading to an unpremeditated production reduction of the affected firms. These can be natural disasters such as earthquakes and volcano eruptions and climate extremes such as heatwaves, floods, and tropical cyclones. For the economic system, the latter are likely to become even more challenging in the future as they are projected to increase in intensity and frequency under ongoing climate change (Herring et al., 2015; Field et al., 2012). In the present-day global economy, local firms and markets are highly interlinked forming a complex network of supply and value chains. In the process of globalization, the density of inter-firm linkages has increased significantly (Maluck and Donner, 2015). In addition, production principles have changed. Lean production schemes have been implemented that permit reducing storage costs, but, at the same time, render sectors more dependent on the timely delivery of intermediate goods needed for production. Consequently, local disasters can have global repercussions. Locally, disasters directly suppress economic activity such as commodity production. The associated losses, however, can spread to other sectors via back- and forward-linkages of the supply chains causing indirect losses (Rose, 2004; Acemoglu et al., 2012). Further, recent studies suggest that in the last decades the vulnerability of the economy with respect to climate extremes has increased (OECD, 2015; Wenz and Levermann, 2016). Overall, indirect losses can represent a significant - or even dominant - share of total losses (Noy, 2009; Przyluski and Hallegatte, 2011). Unfortunately, state-of-the-art integrated assessment models tend to underestimate costs of natural disasters (Revesz et al., 2014), partially because they cannot resolve economic losses resulting from climate extremes appropriately (Stern, 2016).

A profound understanding of the direct and indirect losses of climate-related disasters is also important with respect to a comprehensive assessment of the costs that climate change will impose upon societies, the so-called social costs of carbon. Especially in view of the international agreement to limit global warming "well below $2^{\circ} \mathrm{C}$ above pre-industrial levels" (see, for instance, the discussion in Clark et al. (2016)), reliable estimates of the overall costs of climate change are needed to enable policy makers to develop sound and farsighted plans for climate change mitigation (Rogelj et al., 2015; Robiou du Pont et al., 2016) and adaptation (Cutter et al., 2015). As structural adaptation, supply chains are in need to be rendered climateproof (Levermann, 2014). Unfortunately, state-of-the-art integrated assessment models tend to underestimate climate impact costs (Revesz et al., 2014), partially because they cannot resolve economic losses resulting from climate extremes appropriately (Stern, 2016).

With the acclimate modeling framework, we adopt a global modeling perspective suitable to assess the global repercussions of local disasters. By choosing an agent-based modeling approach, we can account for two aspects essential for the assessment of indirect losses: the heterogeneity of firms (Kirman, 1992; Aoki and Yoshikawa, 2012) as well as for the complex structure of the production network (Battiston et al., 2012; Weisbuch and Battiston, 2007). Together with a high temporal resolution this enables us to resolve the cascading and the absorption of indirect losses along supply chains. For a realistic description of loss mitigation mechanisms, we account for three flexibilities of the economic system that are key for short-term adaptation (Hallegatte, 2014). First, we explicitly model inventories acting as buffer stocks. Second, economic agents in acclimate can shift their demand to non-affected suppliers. Third, firms can adjust their production according to the demand they receive, reducing their production in times of low demand or activating idle capacities in order to increase production in times of high demand. Responding to price signals, firms base the decisions on their optimal production level on clear and simple optimization principles. Finally, non-equilibrium market situations are taken into account. This allows us to describe scarcity situations that arise during the disaster or in the direct aftermath (Hallegatte, 2008): since productive capacities are limited and transportation of goods is time consuming, local supply shortages can not always be mitigated immediately and supply-demand mismatches may occur.

In this paper, we employ our model to analyze the economic response to stylized disasters. The global input-output (I-O) data-set we use as baseline accounts for 27 different sectors (including final demand) on

${ }^{2}$ reached in the United Nations Framework Convention on Climate Change negotiations in Paris in December 2015 
country level. Thus, in this study, firms represent national sectors. By focusing on the outage of a single sector, exemplary the manufacturing sector in Japan, we are able to study indirect effects in a controlled setting. For large outages, indirect losses are found to be in the same order of magnitude as direct losses. By spreading from one sector to the next, they prevail for much longer within the network than the direct ones. Due to non-linearities in the propagation dynamics they can, in fact, even peak long after the direct losses have ceased.

This paper is organized as follows. At first, we review the relevant literature and discuss in how far our model differs from existing approaches in Section 2. Next, we provide an overview of the acclimate model in Section 3. We then analyze local aspects of the economic response dynamics to stylized disasters in Section 4, before discussing the response of the global economy in dependence of disaster duration and disaster size in Section 5. Finally, we discuss our main findings in Section 6, before concluding in Section 7. A detailed and comprehensive description of the numerical model can be found in Appendix A.

\section{Related literature}

For a long time, it was commonly assumed that micro-level idiosyncratic shocks would average out and that their effects on the aggregate macro-level would therefore be negligible (Lucas, 1977). However, only recently, Gabaix (2011) revealed in a ground-breaking study that this is not the case if the distribution of firm-sizes is sufficiently fat-tailed. The author bolstered this 'granular' hypothesis empirically by showing that idiosyncratic movements of US firms make a significant contribution to the observed macroeconomic variations in output growth. Further important theoretical contributions in this direction were made by Acemoglu et al. (2012) and Carvalho (2014), who focused on the impact the topology of the economic network has on shock propagation. They revealed that sizable aggregate fluctuations can result from idiosyncratic shocks if there are 'hubs' in the network, i. e., well connected firms supplying numerous firms of different sectors, which facilitate the cascading of losses from one layer of the supply chains to the next. These theoretical findings were complemented by more empirical ones. Gabaix (2009) revealed that power-laws, i. e., fat-tailed distributions, are ubiquitous in economics, and a study by Arenas et al. (2002) on self-organized criticality in economic networks suggested that economic systems are often at the boundary between chaos and organization - in a regime where fluctuations become important because they can trigger regime transitions. Further, Foerster et al. (2011) and Carvalho (2014) showed that the importance of idiosyncratic shocks has increased since the 'great moderation' in the mid-eighties, i. e., the reduction in the volatility of business cycle fluctuations. Di Giovanni et al. (2014) studied the French firm network affirming the importance of a fat-tailed distribution of firm-sizes and the inter-connectedness of the firm network for micro-shocks to contribute to aggregate fluctuations. Moreover, partially triggered by the financial crises, network theory was applied to study shock propagation in economic networks (Schweitzer et al., 2009; Helbing, 2013) with a focus on systemic risks at financial markets (Battiston et al., 2012; Elliott et al., 2014; Acemoglu et al., 2015).

These static analyses have been complemented by dynamic modeling approaches (Mandel et al., 2015). Two well established - albeit rather different - modeling frameworks are I-O and computable general equilibrium (CGE) models (see van der Veen (2004) and Okuyama and Santos (2014) for a comprehensive introduction and Section 6 for a detailed comparison with our model acclimate). Both approaches can reflect the economic dependencies in high detail (Rose, 2004). However, when it comes to describing and temporally resolving the indirect economic effects of disasters due to the cascading of losses along supply chains - the main focus of this paper - both, I-O and CGE, approaches may not be able to realistically describe the economic responses in the period of days to months following a disaster (Hallegatte, 2008; Farmer and Foley, 2009; Farmer et al., 2015). Whereas the production system in I-O models is fixed rendering short-term adaptation impossible (Albala-Bertrand, 2013), that of CGEs is highly adaptive and flexible due to price responsiveness and a high degree of substitutability among commodities. CGEs are calibrated such that supply and demand elasticities as well as the elasticities of substitution are suitable to describe an economy in long-term equilibrium. Consequently, in contrast to I-O models that tend to overestimate losses, CGEs are prone to mitigate losses unrealistically well (Hallegatte, 2008). 
Attempts to represent a system's complex dynamics from the bottom up are undergone in agent-based models (ABMs), e. g., Gallegati and Richiardi (2011) and Axtell (2007). Here, the stylized facts of macroeconomic systems emerge from the interplay of individual heterogeneous agents (Caiani et al., 2016; Delli Gatti et al., 2005), which may lead to non-equilibrium dynamics. Micro-economically founded agent-based growth models have, for instance, been proven to reproduce exponential growth (Delli Gatti et al., 2007; Mandel, 2012). In recent years, $A B M s$ have been frequently applied to study the implications of specific policies (Dosi et al., 2010). Further, similar to static methods, a focus was put on systemic risk by studying bankrupt avalanches and their dependence on network topology (Weisbuch and Battiston, 2007; Delli Gatti et al., 2010; Riccetti et al., 2013; Chaney, 2016; Wolski and van de Leur, 2016). However, ABMs still struggle to gain broader recognition from the mainstream neoclassical economic community (Leombruni and Richiardi, 2005). In particular, they are criticized for providing the modeler with too much freedom in the implementation of the decision rules for the bounded rational agents - usually, ad-hoc behavioral rules are chosen that appear meaningful and allow to reproduce key stylized facts (Salle, 2015). However, the unambiguousness of the representative, perfectly rational agents in neoclassical macroeconomics is lost (Fagiolo et al., 2007) since different sets of rules may reproduce the same stylized facts. Yet, the agents' decision rationale may be derived from behavioral studies investigating the individual decisions, the interaction of the individuals, and the emerging macro behavior (Assenza et al., 2015).

Regarding the analysis of production loss cascades along supply-chains, ABM approaches appear promising because loss propagation can be very naturally discussed in a setting where the economy is described by heterogeneous interacting agents yielding a production system with well tuneable flexibilities (Stiglitz and Gallegati, 2011). Only recently, Gualdi and Mandel (2016) presented an ABM of an evolutionary network of monopolistically competitive firms, which is able to reproduce important stylized facts of real-world firm networks. For instance, they can allocate the scale-free topology of firm networks to the competition among the firms. Further, as in the static theory (Acemoglu et al., 2012), their model permits to ascribe aggregate volatility to the fat-tailed distribution of firm sizes.

A foray in the description of disaster-induced losses in supply networks was undertaken by Hallegatte (2008) with the introduction of an agent-based dynamic model, the ARIO model. A more recent version of the model accounts for inventories acting as buffer-stock, which are essential for the assessment of indirect losses in the disaster aftermath (Hallegatte, 2014). This model was successfully employed in several empirical disaster impact studies such as Hallegatte (2009), Ranger et al. (2011), and Hallegatte et al. (2011). Further, Henriet et al. (2012) extended the model to study how the robustness of a firm network to micro-shocks depends on the structure of the network as well as the heterogeneity of direct losses. Moreover, the authors provided an algorithm to disaggregate sectoral I-O tables such that a firm network with realistic size distribution is obtained.

The first version of the acclimate modeling framework was introduced by Bierkandt et al. (2014) to study the downstream propagation of production losses in a global supply network in the presence of inventories. The model was then extended to account for adaptation of upstream demand - in terms of quantity and in terms of the redistribution of demand among the supplier base in the disaster aftermath (Wenz et al., 2015). Wenz and Levermann (2016) employed the model to study heat-stress induced production losses in the global supply network. They observed that in recent years the supply network has become more susceptible to loss propagation due to an enhanced interconnectivity of the economy, well in line with the findings of Henriet et al. (2012).

In ABMs designed to describe loss propagation in supply networks, firms most importantly have to make two kinds of decisions: Firstly, rationing decisions with respect to their output if the demand they receive exceeds their productive capacity and, secondly, decisions on the redistribution of their upstream demand among their supplier base to mitigate supply shortages in the disaster aftermath. In the ARIO model and the first two versions of the acclimate model, this is done by reasonable ad-hoc behavioral rules. In both models, output is distributed according to a proportional rationing scheme. In the ARIO-inventory model redistribution of demand is not possible, whereas Wenz et al. (2015) redistribute demand according to a supply-reliability measure combined with a proportionality scheme.

In this paper, we take a different approach with respect to the agents' decision rationale. All relevant decisions are governed by local optimization principles, e. g., firms decide upon their production level by 
profit maximization. We believe that this comprises several advantages. First, using prices as an organization mechanism, we can more easily bridge the gap to the CGE literature. The model setup may be interpreted as a 'natural' extension of the CGE approach to the context of 'myopic' agents, which do not have enough information to reach a market clearing equilibrium in each timestep. Instead, disequilibrium situations arise, where prices differ among agents. In the disaster aftermath, these price differences subsequently ease out over many timesteps. This way, the path of the economy back to market clearing equilibrium is temporally resolved and made explicit. Further, in the disaster math, where disequilibrium conditions dominate (Hallegatte, 2008), the assumption of 'myopic' agents appears to be more realistic than implying market clearance immediately. Second, accounting for price effects becomes important for large scale disasters (Hallegatte, 2008), and, at the same time, opens up the possibility to study welfare impacts of disasters. Third, offer prices provide a means to rank potential new suppliers and compare them to the existing supplier base paving the way towards a flexible network that can restructure in the disaster aftermath. Fourth, profit and cost calculations open up the possibility to include growth dynamics by introducing inter-temporal budgets and investment decisions. These steps towards an agent-based growth model will be undertaken in follow-up papers.

\section{Model description}

In this section, we provide an overview of the dynamic agent-based network model acclimate. First, we discuss its mathematical structure in Section 3.1. Next, we introduce its economic agents, firms and regional consumers in Sections 3.1.1 and 3.1.2, respectively. We then discuss the baseline equilibrium of the economy in Section 3.2, before eventually introducing its response dynamics to local, unanticipated and idiosyncratic production shocks in Section 3.3. Overall, we focus on giving an overview of the model's structure and motivate the underlying modeling assumptions. A detailed mathematical description of the model can be found in Appendix A, and a list of all parameters, exogenous as well as endogenous variables to the model, is provided in Tables B.1, B.2, and B.3, respectively.

\subsection{Model structure}

We consider an economy consisting of firms under monopolistic competition and regional consumers. These economic agents are interlinked by trade flows forming a complex network of supply chains as sketched in Fig. 1. The nodes of this trade network are the economic agents. Their trade relations are represented by weighted, directed links. In each region, we consider two types of agents: firms, each representing one of the different economic sectors located in the region, as well as a consumer representing the region's final demand. The latter accounts for household consumption, governmental spending, and private investments. We label each economic agent by an index-pair $i r$, where the first index $i$ denotes a sector in the set of all sectors $I$ and the second index $r$ specifies a region in the set of all regions $R$. As the model describes anomalies induced by production shocks, its dynamics evolves around a dynamically stable baseline state of the economy. In the remainder of this section, we first discuss, how we derive the latter from multi-regional input-output (MRIO) tables. From there, we describe the model's disequilibrium dynamics and discuss the additional underlying assumptions.

Baseline state. The baseline trade flows connecting the economic agents are derived from MRIO-tables. The flows in these tables are usually given in units of USD/year and thus have to be divided by the number of timesteps per year to obtain the set of baseline flows $\left\{Z_{i r \rightarrow j s}^{*}\right\}_{i, r, j, s}$ in units of USD/timestep. Here, $Z_{i r \rightarrow j s}^{*}$ denotes the monetary flow from firm $i r$ to economic agent $j s$. The superscript $(\cdot)^{*}$ denotes variables in the baseline state. For a firm $j s$, the sum of all outgoing flows determines its baseline production level $X_{j s}^{*} \equiv \sum_{i r} Z_{i r \rightarrow j s}^{*}$, and for a regional consumer $j s$ the sum of all incoming flows determines its baseline consumption level $\mathcal{C}_{i \rightarrow j s}^{*} \equiv \sum_{r} Z_{i r \rightarrow j s}^{*}$.

Next, we introduce the notion of demand requests in order to define the demand side of the baseline state. Since we focus on losses induced via supply shortages, we assume that the economy is demand-driven. Thus, in each timestep $(t-1)$, each economic agent $k u$ decides (i) on the demand $\left\{D_{j s \leftarrow k u}^{(t-1)}\right\}_{j, s}$ (measured in 
USD/timestep) that it addresses to each of its suppliers $\{j s\}$ and (ii) on the corresponding (dimensionless) reservation prices $\left\{n_{j s \leftarrow k u}^{(t-1)}\right\}_{j, s}$, i. e., the prices it is willing to pay. Only afterwards, in the next timestep $(t)$, its suppliers can decide to which extent they are willing to fulfill the received demand. We define the tuple of quantity demanded and reservation price as demand request. As depicted in Fig. 1, supplier $j s$ responds,

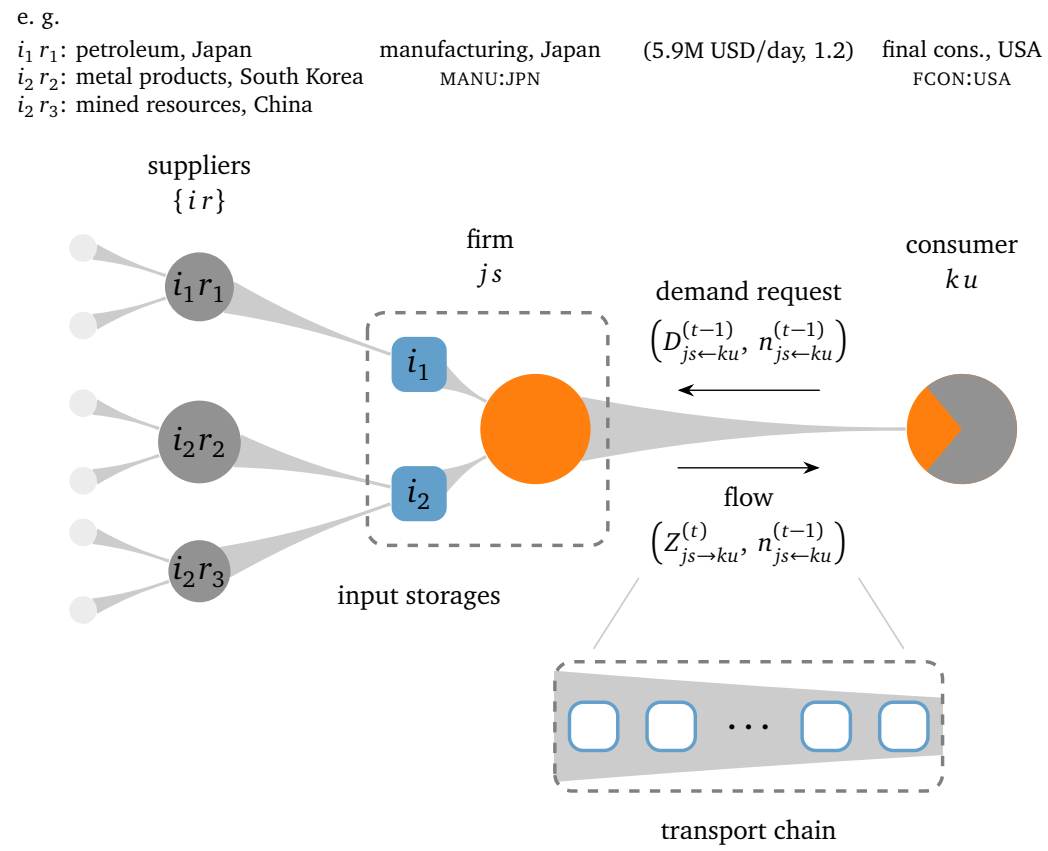

Figure 1: Sketch of the demand-driven economy from the local perspective of a firm. Examples for the sectors and flows under consideration are given above the figure.

in timestep $(t)$, to the demand request $\left(D_{j s \leftarrow k u}^{(t-1)}, n_{j s \leftarrow k u}^{(t-1)}\right)$ it has received from purchaser $k u$ in the previous timestep $(t-1)$ by sending the flow-price tuple $\left(Z_{j s \rightarrow k u}^{(t)}, n_{j s \leftarrow k u}^{(t-1)}\right)$ via the transport chain. Since firms produce at most the demanded quantities, no production-to-stock is possible, i. e., $X_{j s}^{(t)} \leq D_{j s \leftarrow}^{(t-1)} \forall j, s$ holds true, where

$$
D_{j s \leftarrow}^{(t-1)} \equiv \sum_{k u} D_{j s \leftarrow k u}^{(t-1)}
$$

denotes the incoming demand $j s$ receives in timestep $(t)$. We then postulate that, in the baseline state of the economy, each demand is fulfilled, i.e., $D_{j s \leftarrow k u}^{*}=Z_{j s \rightarrow k u}^{*} \forall j, s, k, u$ holds true. This implies that markets clear in the baseline state, i.e., each production sites fulfills its incoming demand $D_{j s \leftarrow}^{*}=X_{j s}^{*} \forall j, s$ and, thus, supply equals demand locally as well as globally. Further, we may deduce from market clearance that (i) there is only one equilibrium (world market) price per commodity, and that (ii) all of a firm's purchasers offer the same reservation price. This permits us to choose the units, in which the commodities are measured, such that the baseline prices for all products are equal. For simplicity, but without loss of generality, we choose a baseline price of 1 USD. In the following, we only discuss prices, denoted by the letter $n$, which are normalized with respect to this value. To allege notation, time indices will be dropped in the following when it is clear from the context to which timestep a variable belongs.

Timing and severity of a disaster are unpredictable for economic actors - at least to a certain extent. In acclimate this is reflected by modeling 'myopic', bounded rational agents. They neither have temporal foresight, nor perfect network oversight since they communicate only with their direct business partners. As already mentioned in the introduction, we aim to resolve the cascading of disaster induced indirect losses 
along supply chains. For short-term loss absorption three flexibilities of the production system appear to be most important: (i) warehousing, (ii) demand adaptation and redistribution, and (iii) idle capacities. We discuss their implementation in acclimate in the following three paragraphs, before explaining in the last two paragraphs of this section why we assume the economy to be demand-driven and the network topology to be static.

Warehousing. For the short-term economic recovery in the disaster aftermath, inventories acting as buffer stocks are key (Hallegatte, 2014). Therefore, in acclimate every agent has input-inventories for the commodities it needs for production or consumption (blue boxes in Fig. 1). Further, since in the last decades lean- and just-in-time production schemes have become established, the commodities 'en route' are nowadays - at least in some sectors - managed as rolling inventory (Shah and Ward, 2007). In acclimate, commodities 'en route' are modeled as transport stock. The number of transport chain links is given by the number of timesteps needed to transport a delivery from supplier to producer (cf. Fig. 1). In each timestep, a delivery is shifted by one transport chain link until it arrives in the corresponding input-inventory of the supplier.

Demand adaptation and distribution. Firms can adapt their upstream demand for input commodities. On the one hand, they can increase it if demand for their product is high or in order to restock their inventories. On the other hand, they can decrease it if (i) their product is less demanded, if (ii) they have to reduce production due to supply shortages of other input commodities that cannot be substituted, or if (iii) they can produce less because they are affected by a disaster. Further, agents can shift their demand from affected to non-affected suppliers as discussed in Section 3.1.1.

Idle capacities. Shifting of demand is most effective if non-affected suppliers have idle capacities that they can activate to meet the increased demand. Empirical evidence for the importance of idle capacities is, for instance, provided by a World Bank report on the Marmara earthquake (World Bank, 1999). Whereas the caused destruction significantly reduced Turkey's Gross Domestic Product (GDP) by $1.5 \%$ to $3 \%$, only relative low production losses were observed. This has been explained with the strong recession that had reduced Turkish GDP by $7 \%$ in the year before the disaster generating idle capacities.

Demand-driven economy. From a modeling perspective, assuming the economy to be demand-driven is consistent with the assumption that economic agents are 'myopic'. To decide if production-to-stock will increase their profit in the long-term, firms would need to form more far-reaching expectations on the development of their incoming demand or the development of the prices for input commodities. For instance, if the firms expected prices for input commodities to rise in the future and demand to remain unchanged, production-to-stock would increase their future profit. Further, we think that neglecting production-to-stock does not significantly reduce the model's performance. Since firms have the possibility to activate idle capacities, they can buffer outages of competitors in the same way as if they had stocks of unsold products. It appears more important to consider stocks at all than to distinguish between input and output stocks.

Static network topology. Moreover, we make the assumption that the supply network is static, i. e., demand can only be shifted between existing connections and no new connections can be established. From a modeling point of view, this aligns well with the assumption that firms have monopolistic markups as discussed in Section 3.2. From an empirical point of view, this is a strong simplification. However, at least in some sectors, high product specialization renders it more difficult for firms to switch to new suppliers in the short-term. Empirical evidence for this hypothesis is, for instance, provided by a study by Boehm et al. (2015) on firms in the US having strong import dependencies to the Japanese economy. The authors found production losses after the 2011 Tōhoku earthquake to be similar to the drop in imports suggesting that firms were not able to replace import commodities by switching to new suppliers in the short-term, i. e., in the months following the disaster. Further anecdotal evidence was given by Carvalho (2014) with respect to the automobile industry in the United States. Another recent example is the production interruption in Volkswagen production plants in 2016 due to a supplier dispute. The firm stopped production in six sites taking important economic losses, because switching to new suppliers was not possible in the short term (Financial Times, 2016). However, it is worthy to note that in the longer-term network evolution provides an important adaptation mechanism, which 
will be addressed in upcoming versions of the acclimate modeling framework. For the purposes of this paper focusing on modeling the direct disaster aftermath, where supply-chain interruptions appear, accounting for inventories and transport times appears to be more important. Also, in the data used, firms and consumers usually have several suppliers per commodity among which they can redistribute their demand to replace affected suppliers in the disaster aftermath.

\subsubsection{Firms}

We model profit-maximizing firms under monopolistic competition. Thus, in each timestep $(t)$, firms decide upon their production level by maximizing profit while respecting constraints imposed by their limited productive capacity and by the limited availability of input commodities. For computational simplicity, each timestep is divided into three subsequent decision points or sub-steps. Profit maximization is assured by applying local optimization principles in each of them. First, firms decide upon their production level by maximizing profit. Second, firms determine the production level that they expect to be profit-maximizing in the next timestep. Afterwards, they communicate this production level and the corresponding offer price to their purchasers to permit them to take a sound decision on how to distribute their upstream demand. Third, after having received these information from their suppliers, firms decide by minimizing purchasing costs (i) how to distribute their own upstream demand and (ii) what their reservation prices are. In the following, these decision points will be referred to as production step, expectation step, and purchasing step, respectively.

Production step. In the production step, each firm determines its profit maximizing production level by taking its limited productive capacity into account. We consider idle capacities in the economy by assuming that each firm $j s$ has the possibility to extend its production above baseline level $X_{j s}^{*}$ by a factor $\beta_{j} \geq 1$, which may vary among sectors. Further, $j s$ 's production level can be reduced by an exogenous factor $\lambda_{j s} \in[0,1]$ representing the disaster's forcing. If no forcing is present, $\lambda_{j s}^{*}=1$ holds true.
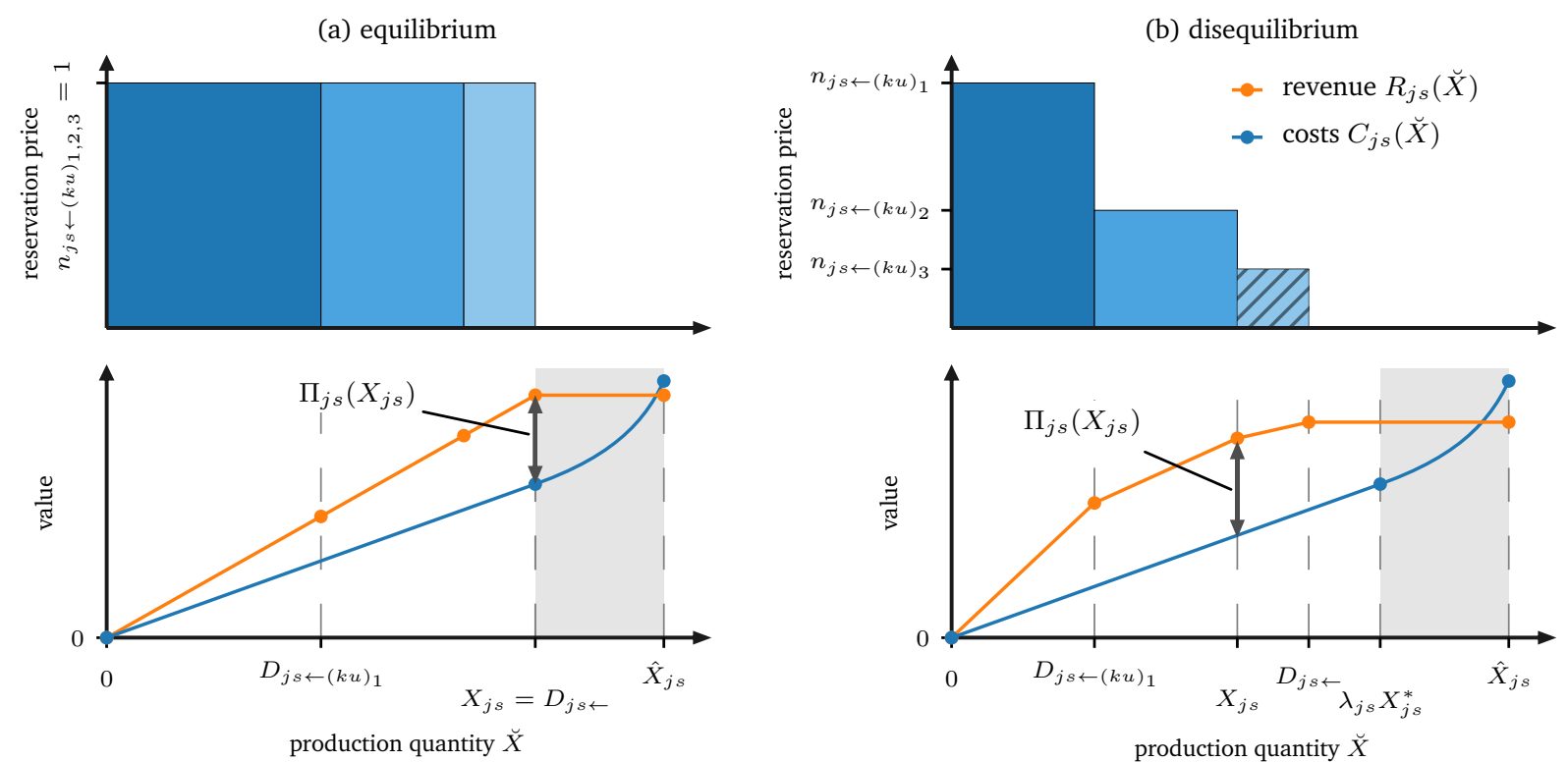

Figure 2: Illustration of how a firm $j s$ determines its profit-maximizing production level $X_{j s}$ (a): in economic equilibrium and (b): in disequilibrium situations arising in the disaster aftermath. Upper panel: Purchaser's reservation prices as a function of cumulative demand. Lower panel: Revenue curves $R_{j s}$ (orange lines) and cost curves $C_{j s}$ (blue lines) as function of $j s$ 's production level under consideration. Gray shadings denote the range of production extension.

At the beginning of the production step, firms receive the demand request their suppliers have issued in the previous timestep. In the following, we note that the monetary value of a demand request $\left(D_{j s \leftarrow k u}, n_{j s \leftarrow k u}\right)$ 
that purchaser $k u$ has issued to supplier $j s$ is given by the product of the demanded quantity and corresponding reservation price,

$$
v\left(D_{j s \leftarrow k u}\right) \equiv n_{j s \leftarrow k u} D_{j s \leftarrow k u} .
$$

From its incoming demand requests a firm $j s$ can derive its revenue curve by first ranking demand requests from high to low reservation prices as depicted in the upper panel of Fig. 2 . The revenue curve $R_{j s}\left(\breve{X}_{j s}\right)$ (cf. solid orange lines in the lower panel of Fig. 2) then describes the functional relationship between the cumulative values of the demand requests and $j s$ 's production level $\breve{X}_{j s}$ (we use the notation $(\cdot)$ to distinguish control variables from the actual values variable assume, i. e., to denote that $j s$ 's revenue is a function of production level, we use the notation $R_{j s}\left(\breve{X}_{j s}\right)$ whereas $X_{j s}$ denotes the actual production level in the present timestep). Thus, if the incoming demand $D_{j s \leftarrow}$ is satisfied, $j s$ cannot increase its revenue further by extending production, and the revenue curve is constant for $\breve{X}_{j s} \geq D_{j s \leftarrow}$.

As in I-O models, we assume that the production function is linear with respect to commodity inputs. All commodity inputs are perfect complements and therefore substitution is not possible among them an assumption that is supported by a recent study by Boehm et al. (2015) suggesting that elasticities of substitution are very low in the months following a disaster. Thus, in the case of supply limitation, the input commodity with the lowest availability limits productive capacity. Prices of input commodities do not depend on the production level, but vary with purchasing costs. Further, we neglect fixed costs for simplicity, but we account for variable production costs of labor, capital depreciation, and variable overhead. Since the latter are usually not contained in MRIO-tables, we do not consider these factors of production explicitly as inputs into the production function, i.e., in our model these factors cannot limit productive capacity ${ }^{3}$. We assume marginal variable costs to be constant up to the baseline production level and to increase linearly above this level to account for extra costs arising for long-hours of workers, etc. In consequence, firm $j s$ 's cost curve may be written as the sum of linear commodity $\operatorname{costs} C_{j s}^{l}$ and variable production $\operatorname{costs} C_{j s}^{v}$,

$$
\mathcal{C}_{j s}\left(\breve{X}_{j s}\right) \equiv C_{j s}^{l}\left(\breve{X}_{j s}\right)+C_{j s}^{v}\left(\breve{X}_{j s}\right) .
$$

Up to the baseline production level, the cost curve increases linearly with production. Above this level, it increases super-linearly due to the nonlinear increase of variable production costs in production extension (see blue lines in the lower panel of Fig. 2).

Firm $j s$ determines its actual production level $X_{j s}$ by maximizing its profit under the constraint that production may not exceed productive capacity $\hat{X}_{j s}$ reading

$$
X_{j s} \equiv \underset{\breve{X}_{j s}}{\operatorname{argmax}}\left[\Pi_{j s}\left(\breve{X}_{j s}\right)\right] \text { subject to } 0 \leq \breve{X}_{j s} \leq \hat{X}_{j s},
$$

where profit is defined as the difference of revenue and costs,

$$
\Pi_{j s}\left(\breve{X}_{j s}\right) \equiv R_{j s}\left(\breve{X}_{j s}\right)-C_{j s}\left(\breve{X}_{j s}\right) .
$$

Note that, in times of crisis, productive capacity can either be reduced by a disaster limiting a firm's ability to produce or by shortages of input commodities.

After production, $j s$ distributes its output among those purchasers with sufficiently high reservation prices. Each purchaser has to pay its reservation price. The reservation prices determine $j s$ 's average production price $\bar{n}_{j s} \equiv \frac{R_{j s}\left(X_{j s}\right)}{X_{j s}}$. In disequilibrium, however, not necessarily all purchasers are served $\left(X_{j s} \leq D_{j s \leftarrow}\right)$. Consequently, $\bar{n}_{j s}$ does not always equal the average reservation price of the purchasers $\bar{n}_{j s}^{p} \equiv \frac{R_{j s}\left(D_{j s \leftarrow}\right)}{D_{j s \leftarrow}}$. Finally, firms put their output into the transport chains, and, at the same time, receive the next deliveries from their suppliers.

\footnotetext{
${ }^{3}$ We are aware that especially not explicitly accounting for a labor market is a restriction of our model because, for instance, disaster impacts on the unemployment rate cannot be described.
} 
Expectation step. After receiving their deliveries, firms know if supply shortages will limit their productive capacity in the next timestep and what their production costs will be. Thus, they can form sound expectations on their upcoming production level and the corresponding average offer price: by forming the 'naive expectation' that (i) the level of external forcing and (ii) their incoming demand will remain unchanged with respect to the current timestep, firms can determine both, expected optimal production level and average offer price by profit maximization as in the production step. They then communicate these quantities together with their expected productive capacity for the next timestep as guidance values to their purchasers.

Purchasing step. At this third decision point, each firm $j s$ first decides upon its total demand for each input commodity $i$,

$$
D_{i \leftarrow j s} \equiv \min \left[\mathcal{E}_{U_{i \rightarrow j s}}+\frac{\Delta S_{i \rightarrow j s}}{\tau_{i \rightarrow j s}}, \mathcal{E}_{D_{i \leftarrow j s}^{j s}}^{\max }\right] .
$$

Here, $\mathcal{E}_{U_{i \rightarrow j s}}$ denotes the amount of commodity $i$ that $j s$ expects to use in the next timestep ${ }^{4}$. This is derived from $j s$ 's expected profit-maximizing production level. Further, $\Delta S_{i \rightarrow j s}$ denotes the deviation from the baseline filling level of $j s$ 's inventory for commodity $i$. In times of scarcity $\left(\Delta S_{i \rightarrow j s}>0\right)$ or abundance $\left(\Delta S_{i \rightarrow j s}<0\right)$, js increases or decreases its demand, respectively. The timescale at which $j s$ aims to balance storage anomalies is given by the parameter $\tau_{i \rightarrow j s}$. Further, the minimum condition in Eq. (5) expresses that demand can be limited by the maximal demand $j s$ expects to be able to source from its suppliers $\mathcal{E}_{D_{i \leftarrow j}^{\max }}^{j s}$ in the next timestep ${ }^{5}$. The latter is derived from the expected productive capacities communicated by $j s^{\prime}$ suppliers in the expectation step.

The expected purchasing costs

$$
\mathcal{E}_{C_{i \rightarrow j s}}\left(\left\{\breve{D}_{i r \leftarrow j s}\right\}_{r}\right) \equiv \underbrace{\sum_{r} \mathcal{E}_{\breve{n}_{i r} s}^{j s}\left(\breve{D}_{i r \leftarrow j s}\right) \breve{D}_{i r \leftarrow j s}}_{\text {expected costs for purchases }}+\underbrace{\mathcal{E}_{C_{i \rightarrow j s}^{\text {pen }}}\left(\left\{\breve{D}_{i r \leftarrow j s}\right\}_{r}\right)}_{\begin{array}{c}
\text { expected additional costs } \\
\text { for transport }
\end{array}},
$$

are a function of the demanded quantities $\left\{\breve{D}_{i r \leftarrow j s}\right\}_{r}$ firm $j s$ addresses to its suppliers and depend on the expected supply curves $\left\{\mathcal{E}_{\tilde{n}_{i r}}^{j s}\right\}_{r}$ of $j s^{\prime}$ s suppliers. Also, transport comes at costs. We assume that transport costs arising in the baseline state are already included in commodity costs, and that extra costs, described by the term $\mathcal{E}_{C_{i \rightarrow j s}^{\text {pen }}}\left(\left\{\breve{D}_{i r \leftarrow j s}\right)\right.$ arise only if the demanded quantities deviate from their baseline values. This can, for instance, occur when means of transportation are not used to capacity. Further, we assume transport costs to increase with the relative deviation of the delivery from its baseline level.

The expected production levels and offer prices communicated by its suppliers permit $j s$ to form expectations on its suppliers' supply curves for the next timestep $\left\{\mathcal{E}_{\tilde{n}_{i r}}^{j s}\right\}_{r}$, i. e., it estimates what price it will have to pay to each of its suppliers for a certain amount of a commodity. To this end, it makes the assumption that if the share it demands from the expected production of a supplier remains unchanged (with respect to the share it has received from the supplier's present production), it has to bid the supplier's offer price. Further, a firm does not expect to be able to crowd out its competitors. Thus, it has to expect to drive the supplier into production extension if it increases its share. In this case, it expects that it must compensate the supplier for the extra costs arising from the higher marginal variable costs in production extension. In the opposite case, where the firm expects to reduce its share, it reduces also its reservation price linearly down to the suppliers production costs for a zero-share.

In line with the local profit maximization in production and expectation steps, firm $j s$ decides upon the optimal distribution of its demand requests among its suppliers by minimizing expected purchasing costs,

\footnotetext{
${ }^{4}$ We use the notation $\mathcal{E}_{(\cdot)}$ to describe the expectation an agent forms at time $(t)$ on the value of its own property $(\cdot)$ in the next timestep $(t+1)$. For instance, $\mathcal{E}_{X_{j s}}$ denotes $j s$ 's expectations at time $(t)$ on its production level in timestep $(t+1)$.

${ }^{5}$ Here, the notation $\mathcal{E}_{(\cdot)}^{(\cdot)}$ denotes the expectation that an agent - indicated by the upper index - makes in timestep $(t)$ on the value of another agent's property in timestep $(t+1)$ - indicated by the lower index. For instance, $\mathcal{E}_{\breve{n}_{i r}}^{j s}$ denotes the expectation that $j s$ has at time $t$ on $i r$ 's supply curve in the next timestep $(t+1)$.
} 
separately for each commodity $i$, under the constraints that (i) cumulative demand $D_{i \leftarrow j s}$ is met, and (ii) individual demand requests must not exceed the amounts $\left\{\mathcal{E}_{D_{i r \leftarrow j s}^{\max }}^{j s}\right\}_{r}$ its suppliers are expected to be able to deliver in the next timestep,

$$
\begin{aligned}
& \left\{D_{i r \leftarrow j s}\right\}_{r} \equiv \underset{\left\{\breve{D}_{i r \leftarrow j s}\right\}_{r}}{\operatorname{argmin}} \mathcal{E}_{C_{i \rightarrow j s}}\left(\left\{\breve{D}_{i r \leftarrow j s}\right\}_{r}\right) \\
& \text { subject to } \sum_{r} \breve{D}_{i r \leftarrow j s}=D_{i \rightarrow j s} \text { and } 0 \leq \breve{D}_{i r \leftarrow j s} \leq \mathcal{E}_{D_{i r \leftarrow j s}^{j s}}^{\max } \forall r .
\end{aligned}
$$

Here, $\mathcal{E}_{D_{i \leftarrow \leftarrow j s}^{\max }}^{\max }$ denotes the maximum value $j s$ expects supplier $i r$ to be able to deliver in the next timestep ${ }^{6}$. The reservation price corresponding to a demanded quantity $D_{i r \leftarrow j s}$ is then given by $n_{i r \leftarrow j s} \equiv \mathcal{E}_{\tilde{n}_{i r}}^{j s}\left(D_{i r \leftarrow j s}\right)$.

\subsubsection{Consumers}

The second type of economic agent considered in acclimate, the consumers, have, in each timestep, to decide (i) upon their consumption level and (ii) upon their demand distribution and corresponding reservation prices. Whereas consumption is done in parallel to the production step of firms, demand requests are distributed during the purchasing step.

Since commodities are perfect complements, a consumer $j s^{7}$ has a separate consumption for each input commodity $i$,

$$
\mathcal{C}_{i \rightarrow j s} \equiv \min \left[\mathcal{C}_{i \rightarrow j s}^{*} \cdot\left(\frac{\bar{n}_{i \rightarrow j s}^{l}}{\bar{n}_{i \rightarrow j s}^{*}}\right)^{\varepsilon_{i \rightarrow j s}^{c}}, \hat{U}_{i \rightarrow j s}\right] .
$$

The consumption for each commodity $i$ varies isoelastically with the corresponding consumer price $\bar{n}_{i \rightarrow j s}^{l}$. This is the price at which $j s$ can currently consume commodity $i$. Further, $\varepsilon_{i \rightarrow j s}^{c} \in\left[-1,0\left[\right.\right.$, and $n_{i \rightarrow j s}^{*}$ denote consumption price elasticity, and the normalized consumer price in the baseline state, respectively. Consumption price elasticities may differ among commodities, which permits to distinguish consumption from investment goods. Consumption goods are needed for immediate consumption and therefore have a lower consumption price elasticity than investment goods. The purchase of investment goods can be delayed if prices are high in the disaster aftermath. Note that, in principle, more complex consumption behaviors could be considered, e. g., in order to account for increased governmental spending subsidizing the sectors most affected by the disaster. Further, the minimum condition in Eq. (8) reflects that consumption may be limited by a reduced availability $\hat{U}_{i \rightarrow j s}$ of commodity $i$ if supply shortages arise in the disaster aftermath.

Having 'naive expectations', consumers assume that their consumer prices for input commodities remain unchanged in the next timestep. For that, they calculate their demand for commodity $i$ by assuming that they will consume (use) the amount $\mathcal{E}_{U_{i \rightarrow j s}} \equiv \mathcal{C}_{i \rightarrow j s}^{*} \cdot\left(\frac{\bar{n}_{i \rightarrow j s}^{l}}{\bar{n}_{i \rightarrow j s}^{*}}\right)^{\varepsilon_{i \rightarrow j s}^{c}}$ in the next timestep. For each commodity, they then calculate their demand as well as the optimal demand distribution from Eqs. (5) and (7), respectively.

\subsection{Baseline equilibrium}

The MRIO-tables provide data of an economy that is not in a (long-term) perfectly competitive equilibrium, in which firms' marginal production costs equal their marginal revenue. Instead, they describe imperfectly competitive markets. In these markets, firms of the same sector differentiate each other not only in price but also by other factors such as existing trade relations ${ }^{8}$, product differentiation, regional tax differences,

\footnotetext{
${ }^{6}$ It is worthy to note that transport costs remain 'virtual'; even if transport costs arise, the firm's demand remains unchanged. Thus, transport costs play the role of a penalty function. They are merely a means to ensure the stability of the baseline equilibrium as discussed in Appendix A.2.3.

${ }^{7}$ Note that in Fig. 1 the regional consumer is denoted by $k u$ because it represents at the same time the purchaser of the firm $j s$.

${ }^{8}$ Note that this assumption aligns well with the assumption of a static network.
} 
and other trade barriers. Accordingly, we assume that, in the baseline state, the economy is in a dynamical monopolistically competitive equilibrium, in which firms have monopolistic markups - as in standard models of monopolistic competition (Ethier, 1982; Romer, 1989).

Whereas monopolistic markups and variable production costs are usually not available, the value added given in acclimate by the sum of commodity costs, variable production costs, and profit - as well as commodity costs can, for the baseline state, be calculated from the MRIO-tables. Thus, we may obtain variable production costs by exogenously specifying firms' monopolistic markups. The latter may vary among firms. Further, data on inventories are usually not provided by MRIO-tables. Therefore, we set inventory levels in the baseline state exogenously. These may vary among firms. Note that due to market clearance, firms do not refer to their inventories in the baseline state. Thus, market clearance implies that the inventory levels in this state constitute the optimal trade-off between preparedness for production disruptions and efficiency in normal times. If this were not the case, firms would empty or replenish their inventory until reaching the profit-maximizing inventory level.

The baseline equilibrium is locally stable which can be understood as follows. First, the baseline production level $X_{j s}^{*}$ maximizes profit. Below $X_{j s}^{*}$, marginal production costs are by the markup smaller than the marginal revenue given by the purchasers' reservation price of unity (see discussion of revenue and cost curves in Appendix A.2.1). Above $X_{j s}^{*}$, the marginal revenue is zero if the firm receives only the baseline level of incoming demand $D_{j s \leftarrow}^{*}=X_{j s}^{*}$ (see flat part of revenue curve (solid orange line) in Fig. 2(a)). In consequence, marginal revenue is smaller than marginal production costs.

Second, the distribution of the demand request in the baseline state $\left\{D_{i r \leftarrow j s}\right\}_{r}$ is cost minimizing. That is because, when deviating from the baseline demand distribution while keeping outgoing demand fixed, a firm has to drive at least one of its suppliers into production extension and demand less from others. Whereas the expected marginal purchasing costs for buying from the former are zero (see details on suppliers' expected supply curves in Appendix A.2.3), the marginal costs for purchasing from the latter decrease with the markup. If the transport penalties are chosen as discussed in Appendix A.2.3, it is guaranteed that the extra costs arising from the marginal penalties overcompensate the decrease in marginal costs, and, in consequence, the baseline equilibrium is locally stable.

\subsection{Dynamics}

In this paper, we discuss the response dynamics of the economic system under local production shocks. Adverse events are modeled as exogenous disturbances temporally reducing the ability of firms to produce. In addition, the economic linkages between agents can be altered to describe impacts on the infrastructure. As discussed above, we want shocks to be not foreseeable for the economic agents. Therefore, the model is solved recursive dynamically and economic agents do not know when they will be forced externally and how long the forcing will subsist.

When a disaster strikes, a disequilibrium state of the economy arises, and, in consequence, production and consumption of the economic agents, and therefore the economic flows may change in time. On the one hand, these perturbations cause supply shortages propagating downstream along the supply chains. On the other hand, they evoke demand anomalies that propagate upstream. If the ability of firms to produce is limited by direct forcing or due to supply shortages in the disaster aftermath, they also reduce their demand for input commodities to avoid an overfilling of their input inventories. This propagation of losses in the opposite direction of the economic flow is also known as backward-ripple effect of the economy (Hallegatte, 2008 , 2014). Both supply and demand anomalies constitute cascading deviations from the baseline state of the network. We aim to study the indirect production and consumption losses they induce.

As already mentioned in the introduction, general equilibrium models, which are widely employed to assess the economic impacts of disasters (Kousky, 2014; Lazzaroni and van Bergeijk, 2014), assume a global equilibrium in each timestep, i. e., they adjust prices to obtain immediate market clearance. In the direct disaster aftermath, these immediately adjusted prices should not be interpreted as real observable prices, but should rather be understood as scarcity indicators (Hallegatte, 2014). In acclimate, we explicitly allow for (local) price anomalies in the disaster aftermath and temporally resolve their decay back to the market clearing equilibrium. If a firm affected by a local disaster has to reduce or stop production, it cannot fulfill 
all the demand it receives, i. e., a local scarcity situation for its product arises. In general, each purchaser perceives a different scarcity of the affected good and is thus offering different reservation prices for it (see Fig. 2(b)). A well-connected agent may be able to easily replace the affected firm by re-directing its demand to its other suppliers of the same good. Thus, its reservation prices are lower than those of a less connected agent. In consequence, reservation prices of different purchasers of the same supplier may differ while supply and demand are unbalanced. Three main drivers determine the timescale of the decay back to market clearing equilibrium: (i) the topology of the economic network, (ii) the ability of the remaining suppliers to mitigate scarcity situations by activating idle capacities, and (iii) the time for storage recovery. If the recovery time is large compared to the timestep, agents refill their inventories slowly, driving their suppliers less into production extension than for smaller values of the recovery time.

\section{Model performance}

In this section, we analyze the response of the model to production interruptions triggered by unexpected adverse events. To this end, we focus on the direct and indirect economic effects of stylized disasters. We study scenarios that are not meant to be realistic but are chosen to illustrate the model performance. The economic network used is based on the Eora-MRIO database (Lenzen et al., 2012) with 2009 as the base year. This permits to account for 27 different sectors including final demand (see Table B.5), and a regional resolution on the country level (see Table B.4). Thus, in this study firms and consumers are represented by national sectors ${ }^{9}$ and country level final demand, respectively. These economic agents correspond to the nodes in the network, which are connected by input and output flows (measured in USD/year). Flows below a threshold value of 1 million USD/year are neglected to avoid numeric instabilities. If this results in agents without in-going connections, then these are removed from the network; likewise firms with negative value added (cf. Eq. (A.23)) are excluded ${ }^{10}$. After this cleanup, the network consists of 4,836 firms and 186 consumers (one for each country) interlinked by about 500,000 connections. The transport times are derived from distances between centroids of the regions. For short distances less than 3,000 kilometers, road transport with an average speed of $35 \mathrm{~km} / \mathrm{h}$ is considered, whereas for longer distances a transport by vessel at $20 \mathrm{~km} / \mathrm{h}$ is assumed ${ }^{11}$. All variables whose baseline values are derived from MRIO-data are listed in Table B.2. Other parameter values used in the numerical simulations are given in Table B.1. Please note that the model is well suited to operate on more refined data depending on the kind of scenario that is to be analyzed $^{12}$. Our model implementation is openly available (Willner and Otto, 2017).

In this paper, we focus on scenarios in which the Japanese manufacturing sector (MANU:JPN) is hit by an unexpected disaster reducing its productive capacity. For our simulation, we choose a daily resolution to model the economic response at the same timescale as the disaster. Note that with the timescale under consideration, also the observed price effects change. The coarser the temporal resolution, the smaller is the observed price volatility. There are three parameters that govern the model's response dynamics with regard to price changes: idle capacities (parameters $\left\{\beta_{i \rightarrow j s}\right\}_{i, j, s}$, cf. Eq. (A.15)), the increase of the firms' marginal variable production costs in production extension (parameters $\left\{\Delta n_{i}^{\text {in, } v,>}\right\}_{i}$, cf. Eq. (A.28)), and the timescales at which agents aim to balance storage anomalies with respect to the chosen timestep (parameters $\left\{\tau_{i \rightarrow j s}\right\}_{i, j, s}$, cf. Eq. (5)). The interplay of all three parameters determines the magnitude of price effects. First, with the amount of idle capacities that can be activated in the economy, the scarcity perceived in the disaster aftermath decreases, and price effects, such as demand surge, become less pronounced. Second, the

\footnotetext{
${ }^{9}$ In the following, we will use the notation of firms and national sectors, interchangeably.

${ }^{10}$ In our modeling setup, this situation may arise if the input commodities of a national sector are in the baseline state more expensive than its produce rendering cost-effective production impossible. Since our model does not consider subsidies, we exclude these national sectors. However, this affects only 11 out of 4,847 national sectors. Hence, the effect of this exclusion on the observed dynamics is expected to be negligible.

${ }^{11}$ The average transport velocities of the different means of transportation have been taken from the Sea Rates project (searates.com).

${ }^{12}$ For future studies, we plan to incorporate spatially refined data using a newly developed refinement algorithm (Wenz et al., 2015), which handles non-homogeneous regional and sectoral resolutions. For instance, the region directly hit by the disaster can be modeled with a high regional resolution to account for small scale disasters and with a high sectoral resolution to account for the heterogeneity of sectors.
} 
magnitude of these price effects is determined by the increase of the firms' marginal variable production costs in production extension. If a short timestep is chosen, we expect this price increase to be larger - in relative terms - than for a longer timestep. This is because, in a short time-frame, it is more expensive for firms to activate idle capacities; the production system is stiffer. Third, the timescale at which agents aim to balance inventory anomalies determines to which extent idle capacities are activated because shorter balancing times imply higher demand (cf. Eq. (5)). If agents aim to balance their inventory anomalies slowly with respect to the chosen timestep, price effects are less pronounced than for the case of rapid balancing of inventories. However, in the former case economic recovery takes many timesteps, whereas it takes only a few in the latter case.

We model the impact of the disaster in a stylized way, which permits us to sketch a clear picture of the underlying dynamics and economic principles. For the duration of the disaster, the productive capacity of MANU:JPN is evenly reduced, and, after the disaster, the full productive capacity is restored immediately. That is, no gradual increase of the productive capacity during the reconstruction phase is considered. Further, we assume that no other national sectors are directly affected. The manufacturing sector in Japan was chosen because it is a major sector in the Japanese economy. Therefore, a complete shutdown of this national sector constitutes a non-marginal shock for the Japanese economy with potential global repercussions. The highly industrialized Japanese economy is strongly interlinked with other national economies rendering it a good paradigm to study the indirect effects of disasters on the global supply network. Furthermore, Japan is highly exposed to natural disasters as, for example, the East Japanese earthquake and the subsequent Tsunami in 2011 (Kajitani and Tatano, 2014) or the Kobe earthquake in 1995 (Okuyama, 2014). This renders supply interruptions caused by natural disasters more probable than in other developed economies.

\subsection{Local production and price dynamics}

In this section, we first concentrate on the local recovery dynamics of Japan's manufacturing sector (MANU:JPN) in the disaster aftermath in Section 4.1.1. Then, we discuss how an economic agent that had strongly depended on MANU:JPN's deliveries before the disaster redistributes its demand for manufacturing among its remaining suppliers in Section 4.1.2.

\subsubsection{Local recovery dynamics of the national sector directly hit by the disaster}

We consider a scenario, where initially the economy is in the monopolistically competitive, locally stable baseline equilibrium, before an unpremeditated production shock reduces MANU:JPN's productive capacity close to zero for three days. The recovery dynamics of key local variables in response to this outage is shown in Fig. 3. Pre-disaster baseline values are marked by horizontal gray dashed lines, and the beginnings of timesteps are denoted by vertical black dashed lines. Figure 3(a) depicts the recovery dynamics of incoming demand, production, and expected production, whereas Fig. 3(b) depicts relative deviations of the corresponding prices from their common baseline value of unity. The timeseries have been shifted to emphasize the timing of events within each timestep. At first (light shading), the national sector receives its incoming demand $D_{\text {MANU:JPN }} \leftarrow$ (see Eq. (A.20)) from its purchasers, which have an average reservation price of $\bar{n}_{\text {MANU:JPN }}^{p}$ (see Eq. (A.39)). Incoming demand and the corresponding average purchasers' reservation price are denoted by gray dashed lines in Fig. 3(a) and (b). Then (medium dark shading), the national sector determines its production level by profit maximization according to Eq. (A.35). Production and average per unit selling price $\bar{n}_{\text {MANU:JPN }}$ (see Eq. (A.38)) are depicted by blue solid lines in Figs. 3(a) and (b)). Eventually (dark shading), the national sector determines its expected production level $\mathcal{E}_{X_{\text {MANU:JN }}}$ (see Eq. (A.43)) and its offer price $\mathcal{E}_{\bar{n}_{\text {MANu:JP }}}$ (see Eq. (A.40)), which are denoted by red dash-dotted lines in Figs. 3(a) and (b), and communicates them to its purchasers.

In the baseline equilibrium state, for $t<0$, markets clear and MANU:JPN's production equals its incoming demand. Since the equilibrium is stable, MANU:JPN expects to have the same production in the next as in the current timestep, and its present production equals the one it expects to have in the next timestep. The disaster strikes at day 0 reducing MANU:JPN's productive capacity close to zero ${ }^{13}$ until day 2 (blue shaded

\footnotetext{
${ }^{13}$ Note that we do not consider a complete shutdown because it constitutes a special case, in which the purchasers do not send any demand requests and the national sector directly hit communicates neither an average selling price nor an offer price.
} 

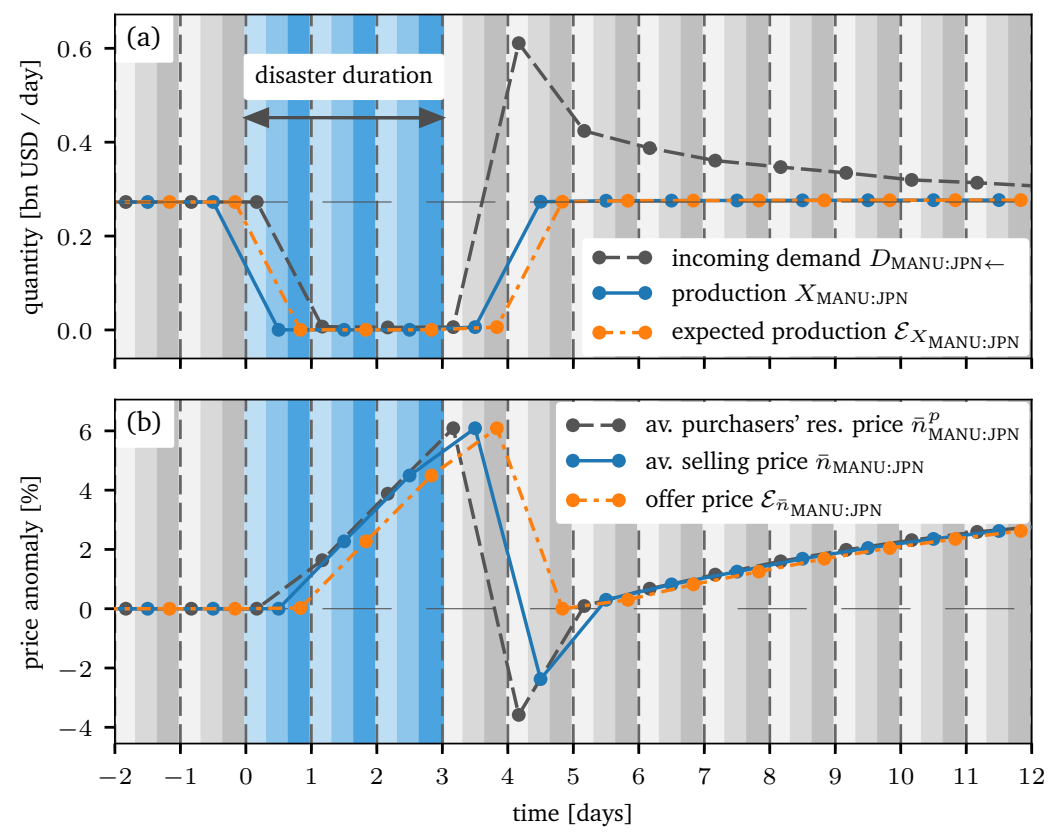

Figure 3: Local recovery dynamics of the manufacturing sector in Japan (MANU:JPN). Parameters: $\lambda_{\text {MANU:JPN }}^{(t)}=0.001$ for $t \in[0,2]$, others as in Table B.1.

areas in Fig. 3), i. e., $\lambda_{\text {MANU:JPN }}^{(t)}=0.001$ for $t \in[0,2]$ (cf. Eq. (A.15)). Since MANU:JPN's purchasers cannot predict the arrival of the disaster, MANU:JPN's incoming demand and the purchasers' reservation prices remain at their baseline levels for this timestep. However, being affected by the disaster MANU:JPN can fulfill only a small share of the incoming demand since its productive capacity $\hat{X}_{\text {MANU:JPN }}-$ and with it its actual production level $X_{\text {MANU:JPN }}$ - is strongly reduced by the external forcing. To calculate its expected production level $\mathcal{E}_{X_{\text {MANU:JPN }}}$ and offer price $\mathcal{E}_{\bar{n}_{\text {MANU:JPN }}}$, MANU:JPN assumes that the incoming demand will remain unchanged in the next timestep (cf. assumption (ii) in Appendix A.2.2). By taking its reduced productive capacity into account, MANU:JPN determines expected production level and offer price by maximizing its expected profit (see Eq. (A.41)). MANU:JPN's purchasers in turn perceive a stock deficit in the transport chains connecting them with MANU:JPN as the deliveries of MANU:JPN are much smaller than in the baseline state (see Eq. (A.46)). Consequently, they try to compensate the shortfall of MANU:JPN by shifting their demand to other business partners. However, they are confronted with transport penalties because MANU:JPN's deliveries are smaller than in the baseline state (cf. Eq. (A.55)). In order to reduce this penalty they offer a higher reservation price to MANU:JPN than in the baseline state aiming to increase their expected shares on MANU:JPN's upcoming production (see Eq. (A.48)). This causes an increase of the average purchasers' reservation price from day 1 to day 3.

When MANU:JPN determines its offer price at day 2, it expects the external forcing to remain unchanged and to still limit its production in the next timestep. However, at day 3, its productive capacity is restored instantly. In response, MANU:JPN's purchasers react to this change by redirecting more demand back to MANU:JPN. Aiming to refill their inventories, they even address more demand to MANU:JPN than in the baseline state. However, they remain with their expected demand shares, in average, below the shares expected to lead to production extension of MANU:JPN (see Eq. (A.51)). In consequence, they offer a reservation price that is smaller than the offer price MANU:JPN has communicated (cf. Eq. (A.53)). Accordingly, at day 4, MANU:JPN receives an above-baseline demand, but the average purchaser's price drops below its baseline level of unity. MANU:JPN responds to the incoming demand by producing more than in the baseline state and by diminishing its monopolistic markup below its baseline value. This causes MANU:JPN's average selling 
price to drop below its baseline value of unity, too. However, since in the calculation of expected production and offer price MANU:JPN respects its baseline monopolistic markup, MANU:JPN's offer price at day 4 only reduces down to its baseline value of unity. In the direct disaster aftermath, from day 4 onward, economic agents that were indirectly affected by the disaster, e. g., by the resulting supply shortages, aim to restock their inventories. Thus, also MANU:JPN perceives a higher incoming demand and is able to sell its production - in average - to higher prices than in the baseline state (see also discussion in Section 4.2).

\subsubsection{Demand redistribution of a national sector indirectly affected by the disaster}

In this section, we consider the same scenario as in the previous section. However, here we discuss how a purchaser of the forced national sector MANU:JPN shifts its demand to unaffected suppliers in order to mitigate MANU:JPN's outage. Figure 4(a) depicts the demand requests that the machinery sector in Hong Kong (MACH:HKG) addresses to its suppliers for manufacturing: Japan (JPN, green shading), the United States of America (USA, orange shading), and others (ROW, blue shading) ${ }^{14}$. In the baseline state, before day 0, MANU:JPN is MACH:HKG's second most dominant supplier for manufacturing. However, in the presence of the disaster, MACH:HKG compensates the (close-to-)outage of MANU:JPN by demanding larger quantities from its remaining suppliers. Additionally, MACH:HKG withdraws from its input inventory (dark gray shading in Fig. 4(b)) at day 2. It determines the optimal distribution of demand requests by minimizing its expected purchasing costs according to Eq. (6). We see from Fig. 4(a) that, from day 2 onward, MANU:JPN needs to source from its inventory but demands larger quantities from its remaining suppliers than in the baseline state. The success of MANU:JPN's purchasing strategy can be deduced from Fig. 4(b) depicting the deliveries of MANU:JPN's suppliers as well as the change in MACH:HKG's storage content; plotted are the contents of the first sections of the transport chains connecting $\mathrm{MACH}: \mathrm{HKG}$ with its suppliers. The anomaly of that inventory content is shown in Fig. 4(c). For instance, at day 0, MACH:HKG tries to compensate for the lack of delivery from MANU:JPN by increasing its demand to its largest supplier, MANU:USA. It even increases its overall demand to compensate for the losses already perceived in the transport chain. However, since the transport time from USA to HKG is 26 days, MACH:HKG has to wait for the additional delivery and starts to resort to its storage at day 2. Accordingly, it keeps its demand to its suppliers (especially to those not directly affected) high to refill its inventory. The distribution slowly returns back to the baseline state after the inventory can successfully be replenished after day 26. Overall, MACH:HKG can keep up its production level (not shown). This indicates that MACH:HKG's strategy for demand redistribution can effectively buffer the close-to-outage of its second largest supplier.

At day 3, directly after the disaster, MANU:JPN communicates to its purchasers that it has recovered from the disaster and regained its full productive capacity. Together with a comparatively low offer price (not shown) this 'persuades' MACH:HKG to request even more from MANU:JPN than in the baseline state (green shaded areas in Fig. 4(a) at day 3 compared to days before day 0). However, since all of MANU:JPN's purchasers respond in this way, MANU:JPN cannot fulfill all demand requests. For instance, MACH:HKG receives less than requested at day 4, because it was outbid by other purchasers of MANU:JPN beforehand. This supply-demand mismatch gradually relaxes until the deficit in the transport chain connecting MANU:JPN and MACH:HKG vanishes and eventually MANU:JPN fulfills the demand request by MACH:HKG like in the baseline state. In consequence, MACH:HKG returns to its baseline demand distribution.

\subsection{Global response dynamics}

In this section, we study the impact that a local production reduction of Japan's manufacturing sector has on the global economy. Figures 5(a), (b) and (c) depict production anomaly, the anomaly of incoming demand, and storage anomaly for the forced national sector (MANU:JPN, blue solid line), the manufacturing sector MANU:ROW aggregated over the rest of the world (without MANU:JPN; gray dashed line), and the global economy without the manufacturing sector (red dash-dotted line), respectively. For simplicity, we

\footnotetext{
${ }^{14}$ It is worthy to note that the outgoing demand requests depicted in Fig. 4(a) are received by the respective suppliers only one timestep later. For instance, the large demand addressed by MACH:HKG to MANU:JPN at day 3 enhances MANU:JPN's incoming demand only at day 4 (see gray dashed line in Fig. 3(a)).
} 

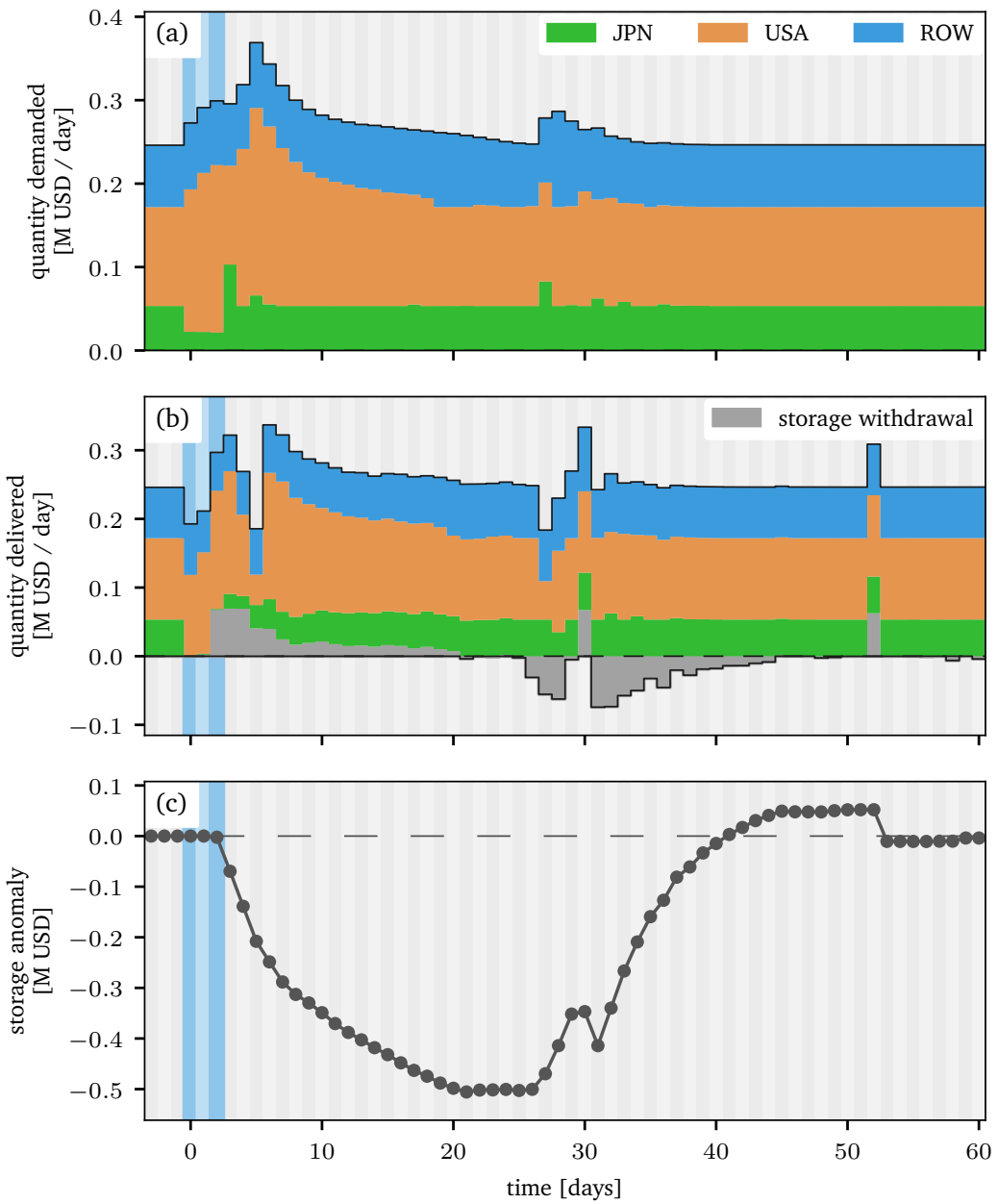

Figure 4: Demand and delivery dynamics in the disaster aftermath. Shown are the demand requests for manufacturing of the machinery producing sector in Hong Kong (MACH:HKG) (a), the corresponding deliveries (b), and the anomaly of MACH:HKG's input storage level for manufacturing goods (c). Parameters as in Fig. 3. 
refer to the latter as the global economy in the following. Anomalies are measured as absolute deviations from their respective baseline values (horizontal gray dashed lines). The storage anomaly is given by the sum of the anomalies of all input storage levels. Figure 5(d) depicts the corresponding relative deviations of MANU:JPN's, MANU:ROW's, and the global economy's average selling prices. Again, the disaster strongly reduces MANU:JPN's ability to produce from day 0 to day 2 (blue shaded areas). Detail enlargements are depicted in Figs. 5(e)-(h) focusing on the timesteps, at which the disaster directly impacts on MANU:JPN, as well as the first few days in the disaster aftermath. Here, subsequent timesteps are marked by alternating light and dark gray shadings.

From Figs. 5(a) and (d), we see that, already during the disaster, MANU:ROW extends its production above the baseline level, revealing that purchasers of MANU:JPN shift demand away from the affected producer towards their remaining manufacturing suppliers driving them into production extension (see enhanced incoming demand of MANU:ROW in Figs. 5(b) and (f)). While the demand is immediately communicated to upstream suppliers, it takes some time until the supplies arrive at their destination. Thus, MANU:Row and the rest of the global economy source from their input inventories to extend production, which reduces their storage levels below their baseline values as depicted in Figs. 5(c) and (g). In contrast, the storage level of the affected national sector MANU:JPN increases as it cannot cancel ordered commodities. In the disaster aftermath, MANU:ROW as well as the rest of the global economy remain in production extension to replenish their inventories, and MANU:JPN is now driven into production extension, too. The timescale of this storage replenishment is either determined by the timescale at which agents aim to replenish their inventories (cf. Eq. (A.44)) or the availability of idle capacities in the economy, depending on which of these constraints is binding.

From the price timeseries depicted in Figs. 5(d) and (h), we gather that price effects decrease in magnitude from the forced national sector MANU:JPN, via MANU:ROW, to the global economy. This can be understood by analyzing the timeseries of the average selling prices in Figs. 5(d) and the corresponding detail enlargement in Fig. 5(h). Locally, the outage of MANU:JPN is a strong perturbation for MANU:JPN's direct purchasers; they have to readdress their demand to their remaining manufacturing suppliers. The commodity manufacturing becomes scarcer, which results in an inflation of its price - demand surge occurs. However, for the global manufacturing sector, and especially for the global economy, the outage of MANU:JPN is a rather small perturbation. This is why global price increases are smaller than local ones.

Concerning production anomalies it is worthy to note from the detail enlargement in Fig. 5(e) that, during the disaster, the production anomaly of the global economy is larger than that of the forced national sector. This implies that the production interruption of MANU:JPN causes further disturbances along the supply chains. Since the input inventories permit firms to sustain the production level of the baseline state for 15 days, these additional production reductions cannot arise from shortages in input commodities, i. e., supply shortages. In contrast, they are induced by a reduction of the demand MANU:JPN addresses to its suppliers. It can be seen from Figs. 5(c) and (g) that the input inventories of MANU:JPN fill up during the disaster. Though it cannot operate, the input quantities it has already ordered before the disaster are delivered successively. As a consequence, MANU:JPN reduces its demand requests to avoid an overfilling of its input inventories. Thus, its suppliers produce less and, in consequence, also have a reduced demand. This results in demand reductions propagating upstream along the supply chains from purchaser to supplier. As mentioned above, this propagation of disturbances in the opposite direction of the economic flows is known as backward-ripple effect (Hallegatte, 2008, 2014).

\section{Importance of indirect losses}

In this section, we focus on the global repercussions of a local, disaster-induced production reduction by discussing direct and indirect production losses as well as loss cascades. Losses are measured in units of USD/day. The direct daily losses are given by the production reductions of the directly affected firms, $\{j s\}_{j, s}$, for the time span of the disaster impact, from day $t=t_{b}$ to day $t=t_{e}$,

$$
l^{D,(t)} \equiv \sum_{m l \in\{j s\}_{j, s}}\left[X_{m l}^{*}-X_{m l}^{(t)}\right] .
$$



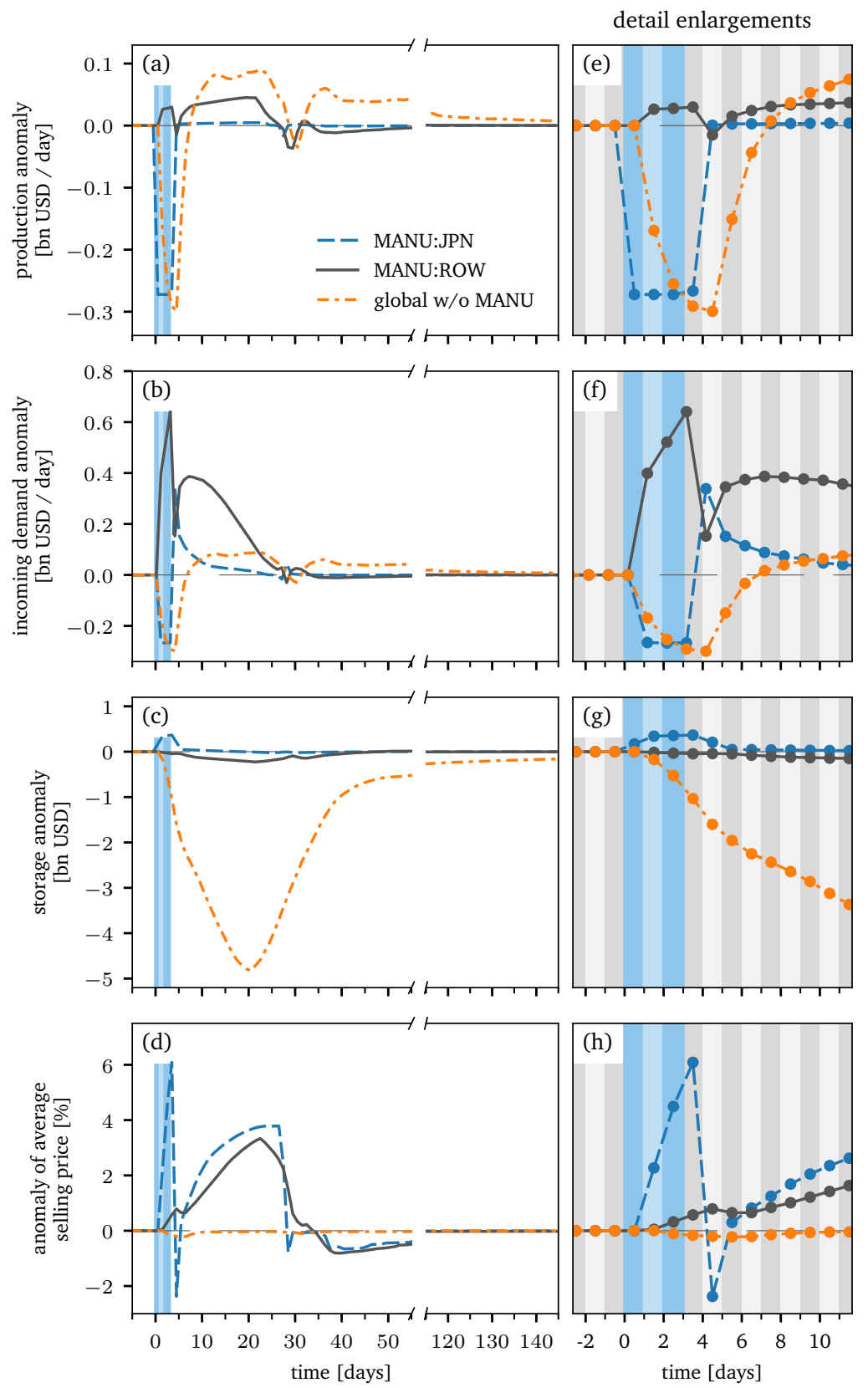

Figure 5: Response dynamics of the manufacturing sector in Japan MANU:JPN, the manufacturing sector aggregated over the rest of the world MANU:ROW (global except MANU:JPN) and the overall global economy without the global manufacturing sector. Parameters as in Fig. 3 . 
Total daily losses are given by the deviation of global production $X^{(t)} \equiv \sum_{m l} X_{m l}^{(t)}$ from its baseline level $X^{*} \equiv \sum_{m l} X_{m l}^{*}$ and therefore read

$$
l^{T,(t)} \equiv X^{*}-X^{(t)} .
$$

Indirect daily losses are then calculated from the difference of total and direct losses,

$$
l^{I,(t)} \equiv l^{T,(t)}-l^{D,(t)} .
$$

Finally, cumulative losses are obtained by subsequently summing daily losses over time, i. e., cumulative direct $L^{D,(t)}$, total $L^{T,(t)}$, and indirect $L^{I,(t)}$ losses read

$$
L^{D, T, I,(t)} \equiv \Delta t \sum_{t^{\prime}=t_{b}}^{t} l^{D, T, I,\left(t^{\prime}\right)} .
$$

Loss cascades occur when direct production losses cannot be buffered by inventories. $1^{\text {st }}$-order cascades of indirect losses arise when the direct purchasers of the forced national sector have to interrupt production because their input inventories are depleted, and they consequently cannot buffer its outage any longer. More generally, loss cascades of $\mathrm{n}^{\text {th }}$-order arise when the forcing is long enough to deplete the input inventories of firms linked to the forced national sector by $n-1$ business partners. These loss cascades were discussed in detail in Bierkandt et al. (2014) describing the first version of the acclimate model, which did not take the demand side of the economy into account. For several reasons, the situation becomes more complex if the demand side is considered as discussed in detail in Wenz et al. (2014). First, in addition to supply shortages, demand shortages may occur leading to backward ripple effects as discussed in Section 4.2. Second, economic agents can readdress their demand to non-affected suppliers. Since, here, the unaffected suppliers have the ability to extend production - the economy has idle capacities - indirect losses can be mitigated effectively. This reduces the risk of supply chain interruptions and therefore increases the economy's resilience. Third, in this model version, firms can reduce or stop production when the average purchasers' price is too low thereby enhancing indirect losses.

At first, we discuss loss propagation in the global supply network in Section 5.1, before studying the dependence of indirect losses upon disaster duration and size in Section 5.2.

\subsection{Propagation of direct and indirect losses}

As in Section 4, we choose a very stylized disaster affecting only one node in the economic network to illustrate the model performance. Again, the production level of MANU:JPN is forced close to zero, but, here, a considerably longer disaster duration of 20 days is chosen $\left(\lambda_{\text {MANU:JPN }}^{(t)}=0.001\right.$ for $\left.t \in[0,19]\right)$. It is now long enough to potentially deplete input inventories of some of MANU:JPN's direct purchasers since these last only for 15 days at baseline production level. As a consequence, loss cascades occur from day 15 onward.

Figure 6(a) shows the temporal evolution of daily total losses (gray solid line and circles), and direct and indirect losses are indicated by blue and red shadings, respectively. Alternating light and dark shadings highlight subsequent timesteps (days), and the onset of $1^{\text {st }}$-order loss cascades is denoted by a vertical black dashed line. Indirect daily losses increase during the first four days of the disaster, then they slightly decrease and almost saturate. Due to the appearance of $1^{\text {st }}$-order loss cascades at day 15 , indirect losses increase significantly until the direct forcing ceases. In the disaster aftermath, indirect losses, and total losses accordingly, become negative indicating that idle capacities are activated to restock inventories. At day 40 losses peak again revealing that supply shortages cannot be buffered completely by the direct purchasers of the forced national sector. Instead, they continue to propagate along the supply chains and peak at bottlenecks. In consequence, the shape of the loss peaks strongly depends on the topology of the underlying trade network and the corresponding transport delays. In summary, it is important to note from Fig. 6(a) that the temporal evolution of indirect and therefore of total losses is strongly nonlinear. Thus, we may conclude that, for a precise loss assessment, it is advantageous to use a model describing the economic impacts on the disaster's timescale. 

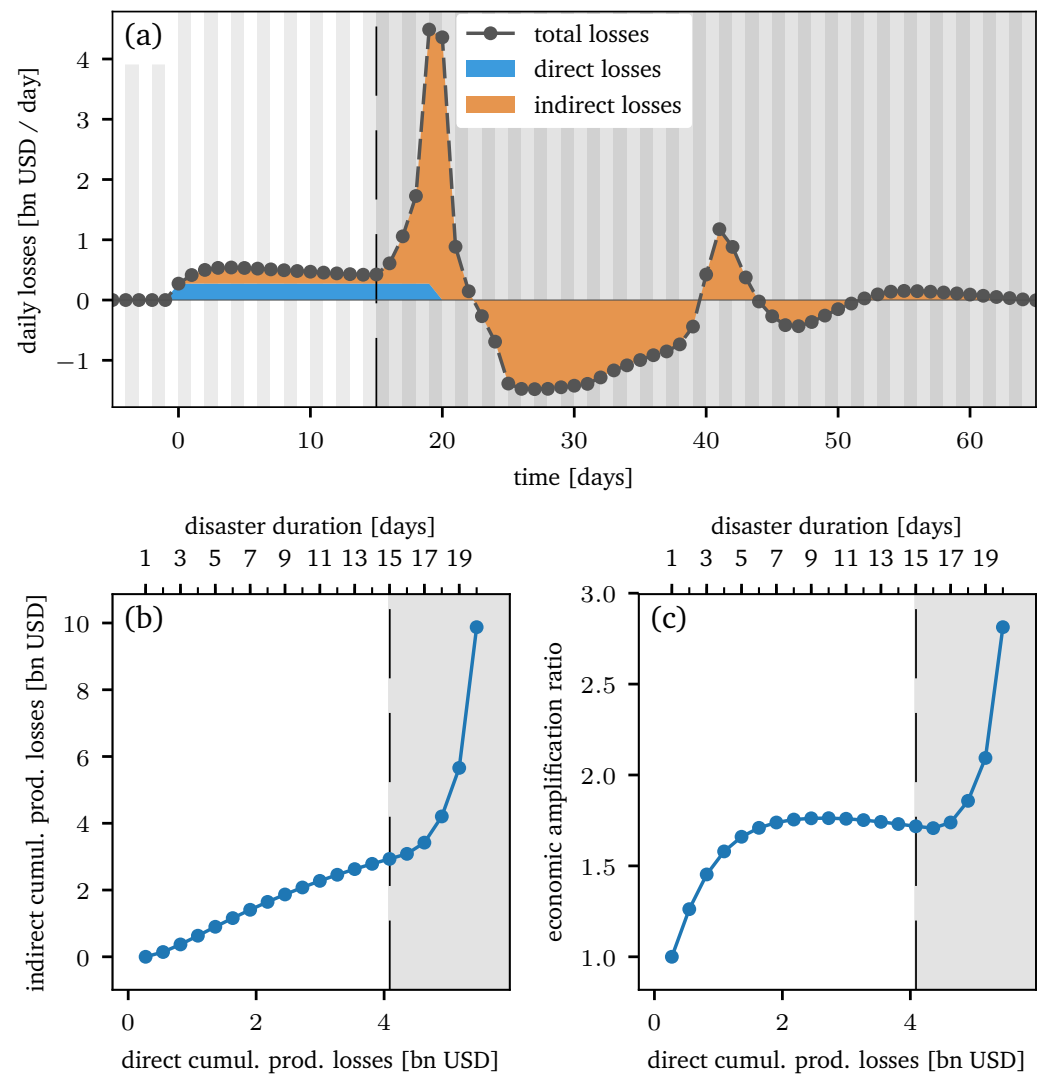

disaster duration [days]

$\begin{array}{llllllllll}1 & 3 & 5 & 7 & 9 & 11 & 13 & 15 & 17 & 19\end{array}$

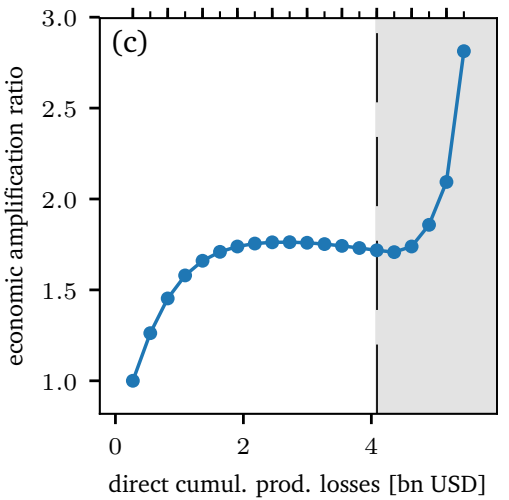

Figure 6: Losses evoked by a strong production reduction of the Japanese manufacturing sector from days 0 to 19 . Dark shaded areas highlight disaster durations for which loss cascades occur. (a): Timeseries of daily production losses. (b): Indirect versus direct cumulative production losses. (c): Ratio of total to direct production loss (economic amplification ratio (EAR)). Parameters: $\lambda_{\text {MANU:JPN }}^{(t)}=0.001$ for $t \in[0,19]$; others as in Table B.1. 
For a better understanding of the relation between direct and indirect losses, Fig. 6(b) shows indirect cumulative losses in terms of direct cumulative losses. Each data-point depicts direct versus indirect losses up to a certain disaster duration (see upper $x$-axis). From day 15 onward (gray shaded area), the first loss cascades occur increasing the slopes of the curves for cumulative losses. We can derive two main messages from Fig. 6(b). First, it reveals that, for non-marginal perturbations of the economy, indirect losses can be of the same order of magnitude as cumulative direct disaster losses, and should therefore be comprised in a comprehensive disaster assessment. Second, inventory holding has a mitigating effect on indirect losses. This is why in the gray shaded area, where inventories are depleted, indirect losses are strongly enhanced.

These two main messages are also underlined by Fig. 6(c) depicting the ratio of total to direct losses, which is plotted as a function of direct cumulative losses and disaster duration. This ratio, known as economic amplification ratio (EAR), was introduced by Hallegatte et al. (2007) representing the factor by which total losses outstrip direct ones. Thus, the EAR is a measure for the importance of indirect losses with respect to direct ones. An EAR significantly larger than unity indicates that direct losses are insufficient to estimate the overall consequences of a disaster (Hallegatte, 2008). It increases rapidly within the first four days of the disaster and then saturates at a value of about 1.6, before increasing again from day 15 onward - in the time-frame where loss cascades occur. This confirms the conclusion of Hallegatte et al. (2007) that indirect losses are important to assess the overall losses of large scale disasters.

\subsection{Dependence of indirect losses upon disaster duration and disaster size}

In this section, the dependence of cascading losses on disaster duration and disaster size is discussed. To ensure comparability with the previous sections, we again consider disasters affecting only the Japanese manufacturing sector (MANU:JPN). Figure 6(a) depicts the timeseries of total losses for close-to-outages $\left(\lambda_{\text {MANU:JPN }}=0.001\right.$ like in the previous sections) of MANU:JPN for different durations. In Figure $6(\mathrm{~b})$ the disaster duration is fixed to 20 days and the disaster size is varied instead, ranging from small to large reductions of productive capacity. To permit better comparability of the system's responses, time is normalized to disaster duration and total losses are normalized to direct ones. This normalization permits us to depict direct losses by gray shaded rectangles in Figs. 7(a) and (b).
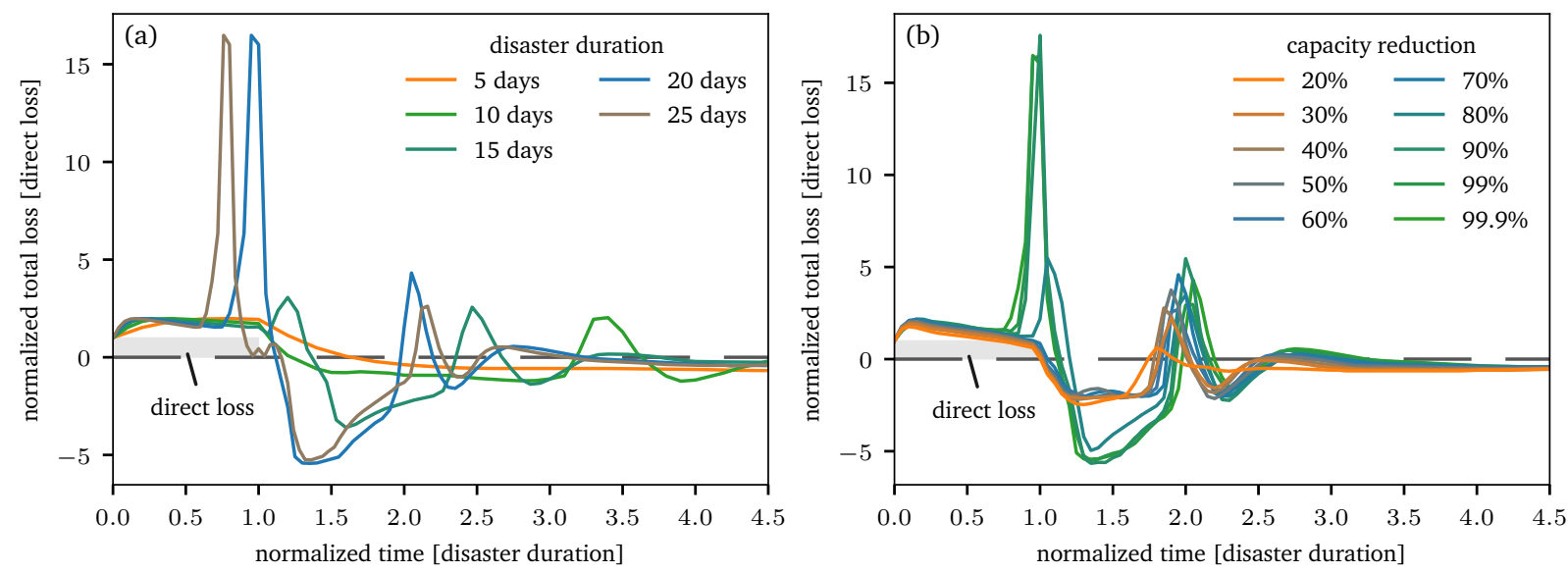

Figure 7: Timeseries of total loss evoked by production reductions of the Japanese manufacturing sector. Time and total losses are normalized with respect to disaster length and direct losses, respectively. The gray rectangle denotes the direct losses. (a): Total losses for close-to-outages of the Japanese manufacturing sector $\left(\lambda_{\text {MANU:JPN }}^{(t)}=0.001\right.$ for $t \in[0,19]$ ) for different durations. (b): Total losses for disasters of different sizes, i. e., different values of $\lambda_{\text {MANU:JPN }}$; disaster duration is fixed to 20 days. Parameters as in Table B.1.

By comparing, losses arising from short disasters with those arising from longer ones in Fig. 6(a), we note that with increasing disaster duration the economy is driven further in production extension. Also, the regime of production extension is entered sooner relative to disaster duration. This is because storage 
deficits increase with disaster duration and agents are therefore willing to increase their reservation prices to replenish their inventories, driving their suppliers further into production extension. Moreover, as already discussed in Section 5.1, we see that large indirect losses occur if inventories of the direct purchaser of MANU:JPN are depleted (disaster duration larger than 15 days) and $1^{\text {st }}$-order loss cascades are triggered. Further, for longer disasters higher-order effects occur well after the disasters have ceased, indicating that supply shortages propagate further downstream in the supply network. This is very pronounced in the timeseries for disaster durations equal to and larger than 10 days. It is worthy to note that these higher-order effects arise already for disasters not long enough to trigger $1^{\text {st }}$-order loss cascades as seen from the disaster of 10 days duration. Thus, we conclude that already relatively small supply disturbance can accumulate at bottlenecks further downstream in the supply network and cause supply disruptions.

Eventually, from Fig. 6(b) depicting the dependence of total losses on the size of direct losses, we note that for small disasters, which are not large enough to trigger $1^{\text {st }}$-order cascades, towards the end of the direct forcing, total losses can even become somewhat smaller than direct losses (see loss timeseries for remaining capacities ranging between $80 \%$ to $30 \%$ ). This reveals that even relatively large capacity reductions can be mitigated efficiently. The local cost minimization enables agents to efficiently activate idle capacities of their suppliers. For larger disasters, however, this loss mitigation mechanism reaches its limit, and loss cascades occur. Overall, Fig. 7 highlights the strongly nonlinear relationship between the size and the duration of direct losses on the one hand, and the size as well as the temporal evolution of total losses on the other. To keep the analysis concise, we here concentrate on a very aggregate view of the whole economy. With the setup of the acclimate model, however, we can also analyze regional differences in loss distribution to be addressed in subsequent studies.

\section{Discussion}

With the acclimate modeling framework, we aimed to tackle some important limitations of other modeling approaches prevalently used for the assessment of indirect disaster effects. In particular we tried to find middle ground between I-O and CGE modeling frameworks with their often opposing assumptions and foci. In the following, we discuss our findings highlighting differences and similarities to these two model types as well as to other ABM approaches.

Spatial and sectoral resolution. Being based on I-O tables, acclimate has been designed to account for a large number of heterogeneous economic agents in order to reflect the economic inter-dependencies in high detail. As in I-O and CGE models, its spatial and sectoral resolution is, in principle, only limited by data availability. Modeling the interplay of multiple heterogeneous agents and considering the network structure of their interlinkages allows to describe complex effects such as cascading losses and lock-in situations (Acemoglu et al., 2012, 2015). Yet, considering national sectors, this study still lacks a realistic representation of the firm size distribution. For a local economy, this was done by Henriet et al. (2012) revealing that indirect losses strongly depend on the topology of the firm network, and an aggregate perspective - as assumed in this study - still tends to underestimate losses. However, often in disaster impact studies only the local economy of the affected region is modeled in detail. Since the supply chain network is globally integrated (Lenzen et al., 2013), and value added chains span the globe (Boehm et al., 2015), this limits the potential of these studies to describe the impacts that local disasters have on the global economy.

Flexibility of the economic system. The amount of indirect losses observed in an economic model is governed by the flexibility of its production system. This is why acclimate aims to find a reasonable balance between the fixed production system in I-O models and the highly flexible one in CGE approaches. We decided to incorporate microfoundations for the agents' behavior. In that, agents have the possibility to respond to their current situation, up-stream by demand re-distribution (cf. Fig. 4), and down-stream by adaptation of their production levels (cf. Figs. 5(a) and (e)). The practical importance of these adaptation mechanisms has been highlighted in a study by van der Veen and Logtmeijer (2005) revealing that an economy's vulnerability with respect to supply interruptions is strongly reduced when demand re-addressing is possible and idle capacities are present. Further, in acclimate, supply disruptions are mitigated by the economic agents' input inventories 
acting as buffer stocks. At the same time, substitution among different input commodities as in CGE models is not possible.

As discussed in Section 4.2, price inflation in the disaster aftermath activates prior idle production capacities in the economic system enabling the agents to restock their inventories. The extent to which warehousing can enhance the resilience of the global economy to local production disruptions was revealed by our analysis of the economic amplification ratio in Section 5.1. We found that the baseline inventory level determines the disaster size that can be absorbed by the economic system. If this threshold is exceeded, indirect losses attain the same order of magnitude as direct losses. These findings are in line with earlier studies by Hallegatte (2014) and MacKenzie et al. (2012) indicating that the interplay of both, inventories and idle capacities, constitutes a powerful strategy for disaster impact mitigation. However, since stock-holding is costly, the chosen inventory level is always a trade-off between economic robustness against production interruptions and efficiency in normal times (Henriet et al., 2012).

Summing up, the production system in acclimate is less rigid than the one of I-O models, but it remains, at the same time, less flexible than the one of CGE models. Our modeling approach is therefore suited best for the timescale of months following a disaster - too short for the economic system to restructure and to substitute scarce commodities, but long enough to adjust its productive capacities. On longer timescales of years, accounting for the restructuring of the economic network in the aftermath of a disaster appears to be important in order to realistically describe the evolution of firm size distributions (Gualdi and Mandel, 2016) or disaster impacts on long-term growth (Mandel, 2012).

Temporal resolution. I-O and CGE modeling frameworks either statically compare pre- and post-disaster states of the economy, or, in the case of dynamic CGEs, have a coarse temporal resolution of 5 to 10 years (Okuyama, 2007). In consequence, scarcity situations arising from supply chain disruptions in the immediate disaster aftermath cannot be temporally resolved, rendering a comprehensive loss assessment difficult. This is why, in acclimate, we opted for a high temporal resolution to study the disaster impacts on the same timescale as the shock occurs, which is in the order of days to months. This permitted us to resolve the cascading of indirect losses and to dynamically detect bottlenecks of the supply network that are responsible for large indirect losses (cf. Fig. 6). Further, acclimate enabled us to systematically study the dependence of cascading losses upon disaster duration and disaster size (cf. Figs.7).

Since real world economic actors have to cope with uncertainties on future events (Babiker et al., 2009), the myopic agents in acclimate provide a more realistic setting for disaster impact analyses than dynamic CGEs with inter-temporal optimization assuming perfect foresight of all economic actors. However, the latter are more favorable to determine optimal policies in the long-run (Chen et al., 2016).

Disequilibrium dynamics. In comparison to I-O models, CGE models have the advantage that they can account for price effects. The representation of prices opens up the possibility to base the agents' decision rationale on clear and simple optimization principles.

There is one further major difference between acclimate and CGE models worthy to discuss. Since the agents in acclimate optimize independently, there is no need to imply a market clearing equilibrium in each timestep as it is done in CGE approaches; in the short-term, disequilibrium situations with local supply-demand mismatches may arise (cf. Fig. 4). In disequilibrium, reservation prices of different purchasers sourcing from the same supplier may differ according to the scarcity each of the purchasers perceives. We find that these differences decrease over time, when the system decays back to the market clearing equilibrium.

\section{Conclusions}

In this paper, we presented the model acclimate, which has been designed to assess the economic impacts of unanticipated production disruptions, caused, for instance, by extreme weather events. Since a comprehensive disaster analysis is beyond the scope of this model description paper, we studied the impact of stylized disasters of different sizes affecting the Japanese manufacturing sector. In our analysis we adopted a global perspective and showed that, in the supply network, disruptions can spread from one national sector to the next causing cascading indirect losses. Over the last decades, firms have increasingly eliminated 
cost inefficiencies by reducing their warehousing and by striving for a smaller supplier base. Our analyses suggest that these trends may have to be reversed in the future if meteorological extreme events are to intensify as projected in a warming world. We find warehousing to be a central adaptation option to reduce indirect losses; a higher redundancy in the supplier base may help to avoid supply shortages. However, more research is needed to provide a sound understanding of the global supply chains' vulnerability in order to enable individual firms to estimate their supply chain risk, and to provide guidelines for risk reduction. Our preliminary analysis suggests that it is crucial to not only focus on first-tier suppliers, but to analyze the supply chain as a whole. Enhancing the resilience of the global supply network cannot be achieved by single countries, but requires an international effort to facilitate the development and implementation of international standards, programs and guidelines to render supply chains climate-proof.

\section{Acknowledgments}

We thank N. Glanemann and T. Heinrich for fruitful discussions. Further, we thank the participants of the session 'Macroeconomic Insights about Climate Change' at the conference Our Common Future 2015, the Complexity Economics Workshop Hamburg 2016, and the session 'Complex Economic Systems and the Role of Institutions' at the Conference on Complex Systems 2016 as well as three anonymous reviewers for helpful and constructive comments. All remaining errors are our own. The work was supported by the Leibniz Competition (SAW-2013-PIK-5) and has received funding from the European Union's Seventh Framework Programme FP7/2007 - 2013 under grant agreement no. 603864, the European Union's Horizon 2020 Framework Program under grant agreement no. 641811, from the Initiative on Extreme Weather and Climate as well as from the Center for Climate and Life of Columbia University, New York City, New York.

\section{References}

Acemoglu, D., Carvalho, V. M., Ozdaglar, A., Tahbaz-Selehi, A., 2012. The Network Origins of Aggregate Fluctuations. Econometrica 80 (5), 1977-2016. 10.3982/ECTA9623.

Acemoglu, D., Ozdaglar, A., Tahbaz-Salehi, A., 2015. Systemic Risk and Stability in Financial Networks. American Economic Review 105 (2), 564-608. 10.1257/aer.20130456.

Albala-Bertrand, J. M., 2013. Disasters and the networked economy. Routledge.

Aoki, M., Yoshikawa, H., 2012. Non-self-averaging in macroeconomic models: a criticism of modern micro-founded macroeconomics. Journal of Economic Interaction and Coordination 7 (1), 1-22. 10.1007/s11403-012-0088-3.

Arenas, A., Díaz-Guilera, A., Pérez, C. J., Vega-Redondo, F., 2002. Self-organized criticality in evolutionary systems with local interaction. Journal of Economic Dynamics \& Control 26 (12), 2115-2142. 10.1016/S0165-1889(01)00025-2.

Assenza, T., Grazzini, J., Hommes, C., Massaro, D., 2015. PQ strategies in monopolistic competition: Some insights from the lab. Journal of Economic Dynamics and Control 50, 62-77. 10.1016/j.jedc.2014.08.017.

Axtell, R. L., 2007. What economic agents do: How cognition and interaction lead to emergence and complexity. The Review of Austrian Economics 20 (2-3), 105-122. 10.1007/s11138-007-0021-5.

Babiker, M., Gurgel, A., Paltsev, S., Reilly, J., 2009. Forward-looking versus recursive-dynamic modeling in climate policy analysis: A comparison. Economic Modelling 26 (6), 1341-1354. 10.1016/j.econmod.2009.06.009.

Battiston, S., Delli Gatti, D., Gallegati, M., Greenwald, B., Stiglitz, J. E., 2012. Liaisons dangereuses: Increasing connectivity, risk sharing, and systemic risk. Journal of Economic Dynamics and Control 36 (8), 1121-1141. 10.1016/j.jedc.2012.04.001.

Bierkandt, R., Wenz, L., Willner, S. N., Levermann, A., 2014. Acclimate-a model for economic damage propagation. Part 1: basic formulation of damage transfer within a global supply network and damage conserving dynamics. Environment Systems and Decisions 34 (4), 507-524. 10.1007/s10669-014-9523-4.

Boehm, C. E., Flaaen, A. B., Pandalai-Nayar, N., 2015. Input Linkages and the Transmission of Shocks: Firm-Level Evidence from the 2011 Tohōku Earthquake. Finance and Economics Discussion Series 2015 (94), 1-73. 10.17016/FEDS.2015.094.

Caiani, A., Godin, A., Caverzasi, E., Gallegati, M., Kinsella, S., Stiglitz, J. E., 2016. Agent based-stock flow consistent macroeconomics: Towards a benchmark model. Journal of Economic Dynamics and Control 69, 375-408. 10.1016/j.jedc.2016.06.001.

Carvalho, V. M., 2014. From Micro to Macro via Production Networks. Journal of Economic Perspectives 28 (4), $23-48$. 10.1257/jep.28.4.23.

Chaney, T., 2016. Liquidity constrained exporters. Journal of Economic Dynamics and Control 72 (March 2005), 141-154. 10.1016/j.jedc.2016.03.010.

Chen, Y.-H. H., Paltsev, S., Reilly, J. M., Morris, J. F., Babiker, M. H., 2016. Long-term economic modeling for climate change assessment. Economic Modelling 52, 867-883. 10.1016/j.econmod.2015.10.023.

Clark, P. U., Shakun, J. D., Marcott, S. A., Mix, A. C., Eby, M., Kulp, S., Levermann, A., Milne, G. A., Pfister, P. L., Santer, B. D., Schrag, D. P., Solomon, S., Stocker, T. F., Strauss, B. H., Weaver, A. J., Winkelmann, R., Archer, D., Bard, E., Goldner, A., Lambeck, K., Pierrehumbert, R. T., Plattner, G.-K., 2016. Consequences of twenty-first-century policy for multi-millennial climate and sea-level change. Nature Climate Change 6 (4), 360-369. 10.1038/nclimate2923. 
Cutter, S. L., Ismail-Zadeh, A., Alcántara-Ayala, I., Altan, O., Baker, D. N., Briceño, S., Gupta, H., Holloway, A., Johnston, D., McBean, G. A., Ogawa, Y., Paton, D., Porio, E., Silbereisen, R. K., Takeuchi, K., Valsecchi, G. B., Vogel, C., Wu, G., 2015. Pool knowledge to stem losses from disasters. Nature 522 (7556), 277-279. 10.1038/522277a.

Delli Gatti, D., Di Guilmi, C., Gaffeo, E., Giulioni, G., Gallegati, M., Palestrini, A., 2005. A new approach to business fluctuations: heterogeneous interacting agents, scaling laws and financial fragility. Journal of Economic Behavior \& Organization 56 (4), $489-512$. 10.1016/j.jebo.2003.10.012.

Delli Gatti, D., Gaffeo, E., Gallegati, M., Giulioni, G., Kirman, A., Palestrini, A., Russo, A., 2007. Complex dynamics and empirical evidence. Information Sciences 177 (5), 1204-1221. 10.1016/j.ins.2006.08.003.

Delli Gatti, D., Gallegati, M., Greenwald, B., Russo, A., Stiglitz, J. E., 2010. The financial accelerator in an evolving credit network. Journal of Economic Dynamics and Control 34 (9), 1627-1650. 10.1016/j.jedc.2010.06.019.

Di Giovanni, J., Levchenko, A. A., Mejean, I., 2014. Firms, Destinations, and Aggregate Fluctuations. Econometrica 82 (4), $1303-1340$. 10.3982/ECTA11041.

Dosi, G., Fagiolo, G., Roventini, A., 2010. Schumpeter meeting Keynes: A policy-friendly model of endogenous growth and business cycles. Journal of Economic Dynamics and Control 34 (9), 1748-1767. 10.1016/j.jedc.2010.06.018.

Elliott, M., Golub, B., Jackson, M. O., 2014. Financial Networks and Contagion. American Economic Review 104 (10), $3115-3153$. 10.1257/aer.104.10.3115.

Ethier, W. J., 1982. National and International Returns to Scale in the Modern Theory of International Trade. American Economic Review 72 (3), 389-405.

Fagiolo, G., Moneta, A., Windrum, P., 2007. A Critical Guide to Empirical Validation of Agent-Based Models in Economics: Methodologies, Procedures, and Open Problems. Computational Economics 30 (3), 195-226. 10.1007/s10614-007-9104-4.

Farmer, J. D., Foley, D., 2009. The economy needs agent-based modelling. Nature 460 (7256), 685-686. 10.1038/460685a.

Farmer, J. D., Hepburn, C., Mealy, P., Teytelboym, A., 2015. A Third Wave in the Economics of Climate Change. Environmental and Resource Economics 62 (2), 329-357. 10.1007/s10640-015-9965-2.

Field, C., Barros, V., Stocker, T., Qin, D., Dokken, D., Ebi, K., Mastrandrea, M., Mach, K., Plattner, G.-K., Allen, S., Midgley, P., 2012. Managing the Risks of Extreme Events and Disasters to Advance Climate Change Adaptation. A Special Report of Working Groups I and II of the Intergovernmental Panel on Climate Change. Tech. rep., IPCC.

Financial Times, 2016. Volkswagen agrees deal to end supplier dispute. URL https://www.ft.com/content/ffd3ee62-6934-11e6a0b1-d87a9fea034f.

Foerster, A. T., Sarte, P.-D. G., Watson, M. W., 2011. Sectoral versus Aggregate Shocks: A Structural Factor Analysis of Industrial Production. Journal of Political Economy 119 (1), 1-38. 10.1086/659311.

Gabaix, X., 2009. Power Laws in Economics and Finance. Annual Review of Economics 1 (1), 255-294. 10.1146/annurev.economics.050708.142940.

Gabaix, X., 2011. The Granular Origins of Aggregate Fluctuations. Econometrica 79 (3), 733-772. 10.3982/ECTA8769.

Gallegati, M., Richiardi, M. G., 2011. Agent based models in economics and complexity. In: Complex Systems in Finance and Econometrics. Springer, pp. 30-53.

Gualdi, S., Mandel, A., 2016. On the emergence of scale-free production networks. Journal of Economic Dynamics and Control 73 , 61-77. 10.1016/j.jedc.2016.09.012.

Hallegatte, S., 2008. An Adaptive Regional Input-Output Model and its Application to the Assessment of the Economic Cost of Katrina. Risk Analysis 28 (3), 779-799. 10.1111/j.1539-6924.2008.01046.x.

Hallegatte, S., 2009. Strategies to adapt to an uncertain climate change. Global Environmental Change 19 (2), $240-247$. 10.1016/j.gloenvcha.2008.12.003.

Hallegatte, S., 2014. Modeling the Role of Inventories and Heterogeneity in the Assessment of the Economic Costs of Natural Disasters. Risk analysis 34 (1), 152-167. 10.1111/risa.12090.

Hallegatte, S., Hourcade, J.-C., Dumas, P., 2007. Why economic dynamics matter in assessing climate change damages: Illustration on extreme events. Ecological Economics 62 (2), 330-340. 10.1016/j.ecolecon.2006.06.006.

Hallegatte, S., Ranger, N., Mestre, O., Dumas, P., Corfee-Morlot, J., Herweijer, C., Wood, R. M., 2011. Assessing climate change impacts, sea level rise and storm surge risk in port cities: a case study on Copenhagen. Climatic Change 104 (1), $113-137$. 10.1007/s10584-010-9978-3.

Helbing, D., 2013. Globally networked risks and how to respond. Nature 497 (7447), 51-59. 10.1038/nature12047.

Henriet, F., Hallegatte, S., Tabourier, L., 2012. Firm-network characteristics and economic robustness to natural disasters. Journal of Economic Dynamics and Control 36 (1), 150-167. 10.1016/j.jedc.2011.10.001.

Herring, S. C., Hoerling, M. P., Kossin, J. P., Peterson, T. C., Stott, P. A., 2015. Explaining Extreme Events of 2014 from a Climate Perspective. Bulletin of the American Meteorological Society 96 (12), S1-S172. 10.1175/BAMS-ExplainingExtremeEvents2014.1.

Kajitani, Y., Tatano, H., 2014. Estimation of roduction Capacity Loss Rate after the Great East Japan Earthquake and Tsunami in 2011. Economic Systems Research 26 (1), 13-38. 10.1080/09535314.2013.872081.

Kirman, A. P., 1992. Whom or What Does the Representative Individual Represent? Journal of Economic Perspectives 6 (2), $117-136$. 10.1257/jep.6.2.117.

Kousky, C., 2014. Informing climate adaptation: A review of the economic costs of natural disasters. Energy Economics 46, 576-592. 10.1016/j.eneco.2013.09.029.

Lazzaroni, S., van Bergeijk, P. A., 2014. Natural disasters' impact, factors of resilience and development: A meta-analysis of the macroeconomic literature. Ecological Economics 107, 333-346. 10.1016/j.ecolecon.2014.08.015.

Lenzen, M., Kanemoto, K., Moran, D., Geschke, A., 2012. Mapping the Structure of the World Economy. Environmental Science \& Technology 46 (15), 8374-8381. 10.1021/es300171x.

Lenzen, M., Moran, D., Kanemoto, K., Geschke, A., 2013. Building Eora: a Global Multi-Region Input-Output Database at High Country and Sector Resolution. Economic Systems Research 25 (1), 20-49. 10.1080/09535314.2013.769938. 
Leombruni, R., Richiardi, M., 2005. Why are economists sceptical about agent-based simulations? Physica A 355 (1), $103-109$. 10.1016/j.physa.2005.02.072.

Levermann, A., 2014. Make supply chains climate-smart. Nature 506 (7486), 27-29. 10.1038/506027a.

Lucas, R. E., 1977. Understanding business cycles. Carnegie-Rochester Conference Series on Public Policy 5, 7-29. 10.1016/01672231(77)90002-1.

MacKenzie, C. A., Santos, J. R., Barker, K., 2012. Measuring changes in international production from a disruption: Case study of the Japanese earthquake and tsunami. International Journal of Production Economics 138 (2), 293-302. 10.1016/j.ijpe.2012.03.032.

Maluck, J., Donner, R. V., 2015. A Network of Networks Perspective on Global Trade. PLOS ONE 10 (7), e0133310. 10.1371/journal.pone.0133310.

Mandel, A., 2012. Agent-based dynamics in the general equilibrium model. Complexity Economics 1, 105-121. 10.7564/12-COEC6.

Mandel, A., Landini, S., Gallegati, M., Gintis, H., 2015. Price dynamics, financial fragility and aggregate volatility. Journal of Economic Dynamics and Control 51, 257-277. 10.1016/j.jedc.2014.11.001.

Noy, I., 2009. The macroeconomic consequences of disasters. Journal of Development Economics 88 (2), $221-231$. 10.1016/j.jdeveco.2008.02.005.

OECD, 2015. Climate Change Risks and Adaptation: Linking Policy and Economics. Tech. rep., OECD. 10.1787/9789264234611-en.

Okuyama, Y., 2007. Economic Modeling for Disaster Impact Analysis: Past, Present, and Future. Economic Systems Research 19 (2), 115-124. 10.1080/09535310701328435.

Okuyama, Y., 2014. Disaster and Economic Structural Change: Case Study on the 1995 Kobe Earthquake. Economic Systems Research 26 (1), 98-117. 10.1080/09535314.2013.871506.

Okuyama, Y., Santos, J. R., 2014. Disaster Impact and Input-Output Analysis. Economic Systems Research 26 (1), 1-12. 10.1080/09535314.2013.871505.

Przyluski, V., Hallegatte, S., 2011. Indirect Costs of Natural Hazards. Tech. rep., SMASH-CIRED.

Ranger, N., Hallegatte, S., Bhattacharya, S., Bachu, M., Priya, S., Dhore, K., Rafique, F., Mathur, P., Naville, N., Henriet, F., Herweijer, C., Pohit, S., Corfee-Morlot, J., 2011. An assessment of the potential impact of climate change on flood risk in Mumbai. Climatic Change 104 (1), 139-167. 10.1007/s10584-010-9979-2.

Revesz, R. L., Howard, P. H., Arrow, K., Goulder, L. H., Kopp, R. E., Livermore, M. A., Oppenheimer, M., Sterner, T., 2014. Global warming: Improve economic models of climate change. Nature 508 (7495), 173-175. 10.1038/508173a.

Riccetti, L., Russo, A., Gallegati, M., 2013. Leveraged network-based financial accelerator. Journal of Economic Dynamics and Control 37 (8), 1626-1640. 10.1016/j.jedc.2013.02.008.

Robiou du Pont, Y., Jeffery, M. L., Gütschow, J., Rogelj, J., Christoff, P., Meinshausen, M., 2016. Equitable mitigation to achieve the Paris Agreement goals. Nature Climate Change 7 (1), 38-43. 10.1038/nclimate3186.

Rogelj, J., Luderer, G., Pietzcker, R. C., Kriegler, E., Schaeffer, M., Krey, V., Riahi, K., 2015. Energy system transformations for limiting end-of-century warming to below $1.5^{\circ} \mathrm{C}$. Nature Climate Change 5 (6), 519-527. 10.1038/nclimate2572.

Romer, P., 1989. Endogenous Technological Change. Tech. Rep. 5, National Bureau of Economic Research, Cambridge, MA. $10.3386 / \mathrm{w} 3210$.

Rose, A., 2004. Economic Principles, Issues, and Research Priorities in Hazard Loss Estimation. In: Okuyama, Y., Chang, S. (Eds.), Modeling Spatial and Economic Impacts of Disasters. Advances in Spatial Science. Springer Berlin Heidelberg, pp. 13-36. 10.1007/9783-540-24787-6_2.

Salle, I. L., 2015. Modeling expectations in agent-based models - An application to central bank's communication and monetary policy. Economic Modelling 46, 130-141. 10.1016/j.econmod.2014.12.040.

Schweitzer, F., Fagiolo, G., Sornette, D., Vega-Redondo, F., Vespignani, A., White, D. R., 2009. Economic networks: the new challenges. Science 325, 422-425. 10.1126/science.1173644.

Shah, R., Ward, P. T., 2007. Defining and developing measures of lean production. Journal of Operations Management 25 (4), $785-805$. 10.1016/j.jom.2007.01.019.

Stern, N., 2016. Current climate models are grossly misleading. Nature 530 (7591), 407-409. 10.1038/530407a.

Stiglitz, J. E., Gallegati, M., 2011. Heterogeneous Interacting Agent Models for Understanding Monetary Economies. Eastern Economic Journal 37 (1), 6-12. 10.1057/eej.2010.33.

van der Veen, A., 2004. Disasters and economic damage: macro, meso and micro approaches. Disaster Prevention and Management: An International Journal 13 (4), 274-279. 10.1108/09653560410556483.

van der Veen, A., Logtmeijer, C., 2005. Economic Hotspots: Visualizing Vulnerability to Flooding. Natural Hazards 36 (1-2), 65-80. 10.1007/s11069-004-4542-y.

Weisbuch, G., Battiston, S., 2007. From production networks to geographical economics. Journal of Economic Behavior \& Organization 64 (3-4), 448-469. 10.1016/j.jebo.2006.06.018.

Wenz, L., Levermann, A., 2016. Enhanced economic connectivity to foster heat-stress-related losses. Science Advances 2, e1501026. 10.1126/sciadv.1501026.

Wenz, L., Willner, S. N., Bierkandt, R., Levermann, A., 2014. Acclimate-a model for economic damage propagation. Part II: a dynamic formulation of the backward effects of disaster-induced production failures in the global supply network. Environment Systems and Decisions 34 (4), 525-539. 10.1007/s10669-014-9521-6.

Wenz, L., Willner, S. N., Radebach, A., Bierkandt, R., Steckel, J. C., Levermann, A., 2015. Regional and Sectoral Disaggregation of MultiRegional Input-Output Tables - a Flexible Algorithm. Economic Systems Research 27 (2), 194-212. 10.1080/09535314.2014.987731.

Willner, S. N., Otto, C., 2017. Acclimate Model. URL https://github.com/acclimate/acclimate. 10.5281/zenodo.853346.

Wolski, M., van de Leur, M., 2016. Interbank loans, collateral and modern monetary policy. Journal of Economic Dynamics and Control 73 (March 2005), 388-416. 10.1016/j.jedc.2016.10.002.

World Bank, 1999. Turkey Marmara Earthquake Assessment. Tech. rep., World Bank. 


\section{Appendix A. Detailed model description}

This Appendix provides a detailed technical description of the acclimate modeling framework. It is written as a comprehensive stand-alone description. First, we introduce the basic model setup in Appendix A.1. Then, we discuss firms and consumers in Appendix A.2 and Appendix A.3, respectively, before explaining the first-order condition for a locally stable baseline equilibrium in Appendix A.4.

\section{Appendix A.1. Basic model setup}

We consider an economy consisting of monopolistic competitive firms and regional consumers. These economic agents are interlinked by trade flows forming a complex network of supply chains as sketched in Fig. 1. The nodes of this trade network are the economic agents and their trade relations are represented by weighted, directed links. In each region we consider two types of agents: firms, each representing one of the different economic sectors located in the region, as well as a consumer representing the region's final demand. The latter accounts for household consumption, governmental spending, and private investments. We label each economic agent by an index-pair $i r$, where the first index $i$ denotes a sector in the set of all sectors $I$ and the second index $r$ specifies a region in the set of all regions $R$.

In the absence of external perturbations, the economy is in a stable monopolistically competitive equilibrium state, the baseline state. Quantities in this state are time constant and are denoted by a superscript $(\cdot)^{*}$. This baseline state can be disturbed by exogenous local disasters, which we define as idiosyncratic production shocks. They cannot be anticipated by the agents. When a disaster strikes, a disequilibrium state of the economy arises, and, in consequence, production and consumption of the economic agents, and therefore the economic flows, may change in time. In general, time-dependent quantities are denoted by a superscript $(\cdot)^{(t)}$ marking the timestep $t \in \mathbb{N}_{0}$ to which they belong.

The baseline trade flows connecting these agents are derived from multi-regional input-output (MRIO) tables. These are usually given in units of USD/year and have, thus, to be divided by the number of timesteps per year to obtain the set of baseline flows

$$
\left\{Z_{i^{\prime} r^{\prime} \rightarrow j^{\prime} s^{\prime}}^{*}\right\}_{i^{\prime}, r^{\prime}, j^{\prime}, s^{\prime}}
$$

where $Z_{i r \rightarrow j s}^{*}$ denotes the monetary flow from firm $i r$ to economic agent $j s$. In principle, the level of regional and sectoral detail of the modeled economy is limited by data availability only. We aggregate these to derive the baseline production level of firm $i r$,

$$
X_{i r}^{*} \equiv \sum_{i^{\prime} r^{\prime}} Z_{i^{\prime} r^{\prime} \rightarrow j s}^{*}
$$

and the baseline consumption level of consumer $j s$,

$$
\mathcal{C}_{i \rightarrow j s}^{*} \equiv \sum_{r^{\prime}} Z_{i r^{\prime} \rightarrow j s}^{*}
$$

We assume a demand-driven economy, which implies that economic agents first decide what demand they address to each of their suppliers and what their reservation prices are. Only afterwards, in the next timestep, their suppliers can decide to which extent they are willing to fulfill the received demand. More precisely, a demand request a purchaser $j s$ addresses to a supplier ir is a tuple $\left(D_{i r \leftarrow j s}^{(t-1)}, n_{i r \leftarrow j s}^{(t-1)}\right)$ of demanded quantity $D_{i r \leftarrow j s}^{(t-1)}$ and corresponding dimensionless reservation price

$$
n_{i r \leftarrow j s}^{(t-1)} \equiv \frac{P_{i r \leftarrow j s}^{(t-1)}}{P^{*}},
$$

which is obtained by normalizing the offered price $P_{i r \leftarrow j s}^{(t-1)}$ with respect to the baseline price $P^{*}$. The monetary value of such a tuple is given by the product of the demanded quantity and its dimensionless price ${ }^{15}$,

\footnotetext{
${ }^{15}$ In the baseline state, the value of each demand request equals the demanded quantity, i. e., $v\left(D_{i r \leftarrow j s}^{*}\right)=D_{i r \leftarrow j s}^{*}$, since price normalization in Eq. (A.4) implies $n_{i r \leftarrow j s}^{*}=1 \forall i, r, j, s$.
} 


$$
v\left(D_{i r \leftarrow j s}^{(t-1)}\right) \equiv n_{i r \leftarrow j s}^{(t-1)} D_{i r \leftarrow j s}^{(t-1)} .
$$

Note that, in the following, we denote values of flows and values of stocks by $v(\cdot)$ and $V(\cdot)$, respectively. Supplier ir responds to $j s$ 's demand requests by sending a flow $Z_{i r \rightarrow j s}^{(t)}$ at price $n_{i r \leftarrow j s}^{(t-1)}$ in the next timestep $(t)$ (see Fig. 1). It cannot negotiate the price, but only decides to which extent it is willing to fulfill the demand request at that price. Since we postulate a demand-driven economy, supply flows must not exceed demand flows. The model is constructed such that the baseline state of the economy is a monopolistically competitive market clearing equilibrium, where supply flows equal demand flows,

$$
Z_{i r \rightarrow j s}^{*}=D_{i r \leftarrow j s}^{*} \forall r, i
$$

Besides flows, the model accounts for two types of commodity stocks acting as buffers under supply shocks: the rolling inventory (see 'transport chain' in Fig. 1) and inventories for the agents' input commodities (blue boxes in Fig. 1) to be discussed in the following.

Rolling inventories. Transport of commodities from producers to purchasers can be time consuming; the commodities 'en route' form the rolling inventory. Let $d_{i r \rightarrow j s} \in \mathbb{N}$ denote the number of timesteps needed for the shipping of commodity $i$ form producer $i r$ to purchaser $j s$. Then $d_{i r \rightarrow j s} \Delta t$ is the time needed for that transport. We conceptualize the commodities on the way as a transport chain with $d_{i r \rightarrow j s}$ transport sections ${ }^{16}$ (see Fig. 1). Then, for $d \in\left\{0, \ldots, d_{i r \rightarrow j s}-1\right\}$ the amount of commodity $i$ that is, at time $t$, contained in section $d$ of the transport chain from ir to $j s$ is given by $\Delta t Z_{i r \rightarrow j s}^{(t-d)}$. Summing the commodities in the transport boxes along the transport chain then yields the rolling inventory for this business connection, which may be written as

$$
T_{i r \rightarrow j s}^{(t)} \equiv \Delta t \sum_{d^{\prime}=0}^{d_{i r \rightarrow j s}-1} Z_{i r \rightarrow j s}^{\left(t-d^{\prime}\right)} .
$$

Further, the total rolling inventory of $j s$ for commodity $i$ is obtained by adding up the rolling inventories of $j s$ 's suppliers for commodity $i$, yielding

$$
T_{i \rightarrow j s}^{(t)} \equiv \sum_{r^{\prime}} T_{i r^{\prime} \rightarrow j s}^{(t)}
$$

Input inventories. Besides the rolling inventory, the economic agents employ input inventories for the commodities that they need for production or consumption to buffer supply failures. Let $S_{i \rightarrow j s}^{(t)}$ denote the content of agent $j s$ 's inventory (or 'storage') for input commodity $i$. It varies with the difference of the input flow $I_{i \rightarrow j s}^{(t-1)}$ and the use $U_{i \rightarrow j s}^{(t-1)}$ of commodity $i$ in the previous timestep.

The input flow $I_{i \rightarrow j s}^{(t)}$ is calculated by summing up the flows that arrive in the current timestep,

$$
I_{i \rightarrow j s}^{(t)} \equiv \sum_{r^{\prime}} Z_{i r^{\prime} \rightarrow j s}^{\left(t-\left(d_{i r^{\prime} \rightarrow j s}-1\right)\right)}
$$

and the value of $I_{i \rightarrow j s}^{(t)}$ is given by

$$
v\left(I_{i \rightarrow j s}^{(t)}\right) \equiv \sum_{r^{\prime}} v\left(Z_{i r^{\prime} \rightarrow j s}^{\left(t-\left(d_{i r^{\prime} \rightarrow j s}-1\right)\right)}\right)=\sum_{r^{\prime}} n_{i r^{\prime} \leftarrow j s}^{\left(t-d_{i r^{\prime} \rightarrow j s}\right)} Z_{i r^{\prime} \rightarrow j s}^{\left(t-\left(d_{i r^{\prime} \rightarrow j s}-1\right)\right)} .
$$

In the baseline state, the input flow $I_{i \rightarrow j s}^{*}$ of each commodity $i$ equals its use in the production process $U_{i \rightarrow j s}^{*}$, i. e., we have

\footnotetext{
${ }^{16}$ This description also permits us to study transport disturbances by damaged or destroyed infrastructure in later model versions.
} 


$$
I_{i \rightarrow j s}^{*} \equiv U_{i \rightarrow j s}^{*} \equiv \sum_{r^{\prime}} Z_{i r^{\prime} \rightarrow j s}^{*}
$$

The storage content in the baseline state is assumed to be a multiple of the baseline input flow $I_{i \rightarrow j s}^{*}$,

$$
S_{i \rightarrow j s}^{*} \equiv \Psi_{i} I_{i \rightarrow j s}^{*}
$$

From Eq. (A.12), we note that, since $I_{i \rightarrow j s}^{*}=U_{i \rightarrow j s}^{*}$ holds true in the baseline state, the agents only have to refer to their input inventories if supply shortages occur in the aftermath of a disaster. The factor $\Psi_{i}$ describes the number of days that $j s$ can keep up its baseline production level if the supply with input commodity $i$ is interrupted.

Further, baseline storage content may be exceeded at most by a factor $\omega_{i}$. This implies that the maximum storage content may be written as

$$
S_{i \rightarrow j s}^{\max } \equiv \omega_{i} S_{i \rightarrow j s}^{*} .
$$

Additionally, we employ the factor $\mu_{i \rightarrow j s}^{(t)} \in[0,1]$ describing the impact of a perturbation reducing the maximum storage capacity. In absence of any forcing, we have $\mu_{i \rightarrow j s}^{(t)}=\mu_{i \rightarrow j s}^{*}=1$. The total inventory is then capped by the maximum capacity $\mu_{i \rightarrow j s}^{(t)} S_{i \rightarrow j s}^{\max }$ and its content at time $t$ is given as

$$
S_{i \rightarrow j s}^{(t)} \equiv \min \left[\Delta t\left(I_{i \rightarrow j s}^{(t-1)}-U_{i \rightarrow j s}^{(t-1)}\right)+S_{i \rightarrow j s}^{(t-1)}, \mu_{i \rightarrow j s}^{(t)} S_{i \rightarrow j s}^{\max }\right] .
$$

We can rewrite this equation as

$$
S_{i \rightarrow j s}^{(t)} \equiv \Delta t\left[r_{i \rightarrow j s}^{(t)} I_{i \rightarrow j s}^{(t-1)}-U_{i \rightarrow j s}^{(t-1)}\right]+S_{i \rightarrow j s}^{(t-1)},
$$

where $r_{i \rightarrow j s}^{(t)} \in[0,1]$ describes the share of the last input flow $I_{i \rightarrow j s}^{(t-1)}$ that could be stored given storage limitations, i. e.,

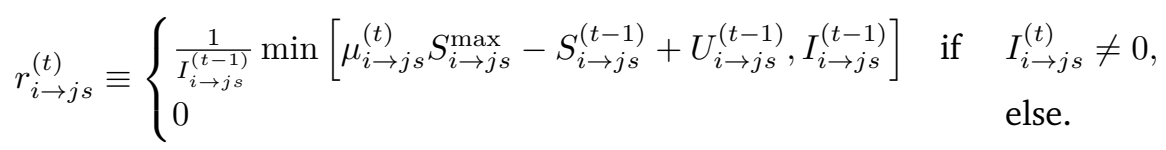

Then, the value $V\left(S_{i \rightarrow j s}^{(t)}\right)$ of the storage content $S_{i \rightarrow j s}^{(t)}$ at time $(t)$ can be calculated as

$$
\begin{aligned}
V\left(S_{i \rightarrow j s}^{(t)}\right) & \equiv \Delta t\left[r_{i \rightarrow j s}^{(t)} v\left(I_{i \rightarrow j s}^{(t-1)}\right)-v\left(U_{i \rightarrow j s}^{(t-1)}\right)\right]+V\left(S_{i \rightarrow j s}^{(t-1)}\right) \\
& =\Delta t \sum_{t^{\prime}=1}^{t-1}\left[r_{i \rightarrow j s}^{(t)} v\left(I_{i \rightarrow j s}^{\left(t^{\prime}-1\right)}\right)-v\left(U_{i \rightarrow j s}^{\left(t^{\prime}-1\right)}\right)\right]+S_{i \rightarrow j s}^{*}
\end{aligned}
$$

where we have employed that in the baseline state $V\left(S_{i \rightarrow j s}^{*}\right)=S_{i \rightarrow j s}^{*} \forall i, j, s$ holds true.

This permits us to calculate the costs to which $j s$ can use input good $i$. These are given by the weighted average of the unit costs of products arriving in the current timestep $v\left(I_{i \rightarrow j s}^{(t)}\right) / I_{i \rightarrow j s}^{(t)}$ and the unit costs of commodities stored in the input inventory at the beginning of the timestep $V\left(S_{i \rightarrow j s}^{(t-1)}\right) / S_{i \rightarrow j s}^{(t-1)}$. Thus, we may calculate these unit costs as

$$
\bar{n}_{i \rightarrow j s}^{l,(t)} \equiv \frac{r_{i \rightarrow j s}^{(t)} v\left(I_{i \rightarrow j s}^{(t)}\right) \Delta t+V\left(S_{i \rightarrow j s}^{(t-1)}\right)}{r_{i \rightarrow j s}^{(t)} I_{i \rightarrow j s}^{(t)} \Delta t+S_{i \rightarrow j s}^{(t-1)}},
$$

This equation has two important implications. First, since $v\left(I_{i \rightarrow j s}^{(t)}\right)$ as well as $V\left(S_{i \rightarrow j s}^{(t-1)}\right)$ depend on the purchasing price of commodity $i$ in the last timestep, the input inventory acts also as a buffer for the unit 
costs $\bar{n}_{i \rightarrow j s}^{l,(t)}$. Even if $j s$ has to pay a high price for the purchase of commodity $i$ in one timestep, unit costs $\bar{n}_{i \rightarrow j s}^{l,(t)}$ will, in general, not increase abruptly. Second, unit costs $\bar{n}_{i \rightarrow j s}^{l,(t)}$ are calculated only on the basis of commodities that are actually available for firm $j s$, and commodities that are still in the transport chain are not considered. It is worthy to note that, in the baseline state, $\bar{n}_{i \rightarrow j s}^{l, *}=1 \forall i, j, s$ holds true. Further, the value of the use $U_{i \rightarrow j s}^{(t)}$ is then given by

$$
v\left(U_{i \rightarrow j s}^{(t)}\right) \equiv \bar{n}_{i \rightarrow j s}^{l,(t)} U_{i \rightarrow j s}^{(t)} .
$$

Finally, the possible use of commodity $i$, i. e., the maximum amount of $i$ that the agent can use for production or consumption, in the current timestep is obtained from $I_{i \rightarrow j s}^{(t)}$ and $S_{i \rightarrow j s}^{(t)}$ as

$$
\hat{U}_{i \rightarrow j s}^{(t)} \equiv I_{i \rightarrow j s}^{(t)}+\frac{S_{i \rightarrow j s}^{(t)}}{\Delta t} .
$$

Appendix A.2. Firms

We model profit maximizing firms under monopolistic competition. Thus, in each timestep, firms decide upon their production level by maximizing profit while respecting constraints imposed by the limited availability of input commodities and their limited productive capacity. For computational simplicity, each timestep is divided into three subsequent decision points or sub-steps. Profit optimization is assured by applying local optimization principles in each of them. In each sub-step, firms exchange information with their business partners, which they need for making decisions in the following sub-step. Figure A.8 depicts the mutual dependencies of variables within one timestep. The three sub-steps are marked by different shadings.

First, in the production step (blue shading in Fig. A.8), firms decide on their production level by maximizing profit. Second, in the expectation step (green shading in Fig. A.8), firms determine the production level that they expect to be profit-maximizing in the next timestep by maximizing expected profit, and third, in the purchasing step (red shading in Fig. A.8), firms decide how to distribute their own upstream demand and what their reservation prices are by minimizing purchasing costs. Production, expectation, and purchasing steps will be discussed in Appendix A.2.1, Appendix A.2.2, and Appendix A.2.3, respectively. To allege notation, in the following, time indices $(t)$ belonging to quantities of the current timestep $(t)$ are suppressed along with time indices $(t-1)$ belonging to demand requests of the previous timestep.

\section{Appendix A.2.1. Production step}

This section provides details of the production step. At first, we discuss how a firm $j s$ determines its productive capacity. Then we describe the firm's revenue curve $R_{j s}$ and its cost curve $C_{j s}$, before deriving an analytic formula for $j s$ 's profit-maximizing production level $X_{j s}$ (cf. Eq. (3)).

Productive capacity. Similar to I-O models, we assume that the production function is linear with respect to commodity inputs. All commodity inputs are perfect complements and therefore no substitution is possible among them. Thus, in the case of supply limitation, the input commodity $i$ with the lowest quantity available for production, $\hat{U}_{i \rightarrow j s}$ (see Eq. (A.14) for its definition), determines the production of firm $j s$. Reducing this quantity by a certain factor then reduces the productive capacity $\hat{X}_{j s}$ by the same factor (constant returns to scale). Further, we assume that a firm $j s$ has the possibility to extend its production above the baseline level $X_{j s}^{*}$ by a factor $\beta_{j} \geq 1$, which may vary among sectors. Moreover, $j s$ 's production level can be reduced by an exogenous factor $\lambda_{j s} \in[0,1]$ representing the disaster's forcing; in the baseline state, no forcing is present, i. e., $\lambda_{j s}^{*}=1$. In consequence, productive capacity

$$
\hat{X}_{j s} \equiv \min \left[\min _{i^{\prime}}\left[\frac{\hat{U}_{i^{\prime} \rightarrow j s}}{U_{i^{\prime} \rightarrow j s}^{*}}\right], \lambda_{j s} \beta_{j}\right] X_{j s}^{*}
$$

is constrained by $j s$ 's maximum production ratio $\lambda_{j s} \beta_{j}$ and by the minimum relative availability of its input commodities $i$. The latter is the lowest ratio of the available quantity $\hat{U}_{i \rightarrow j s}$ and the quantity used in the 


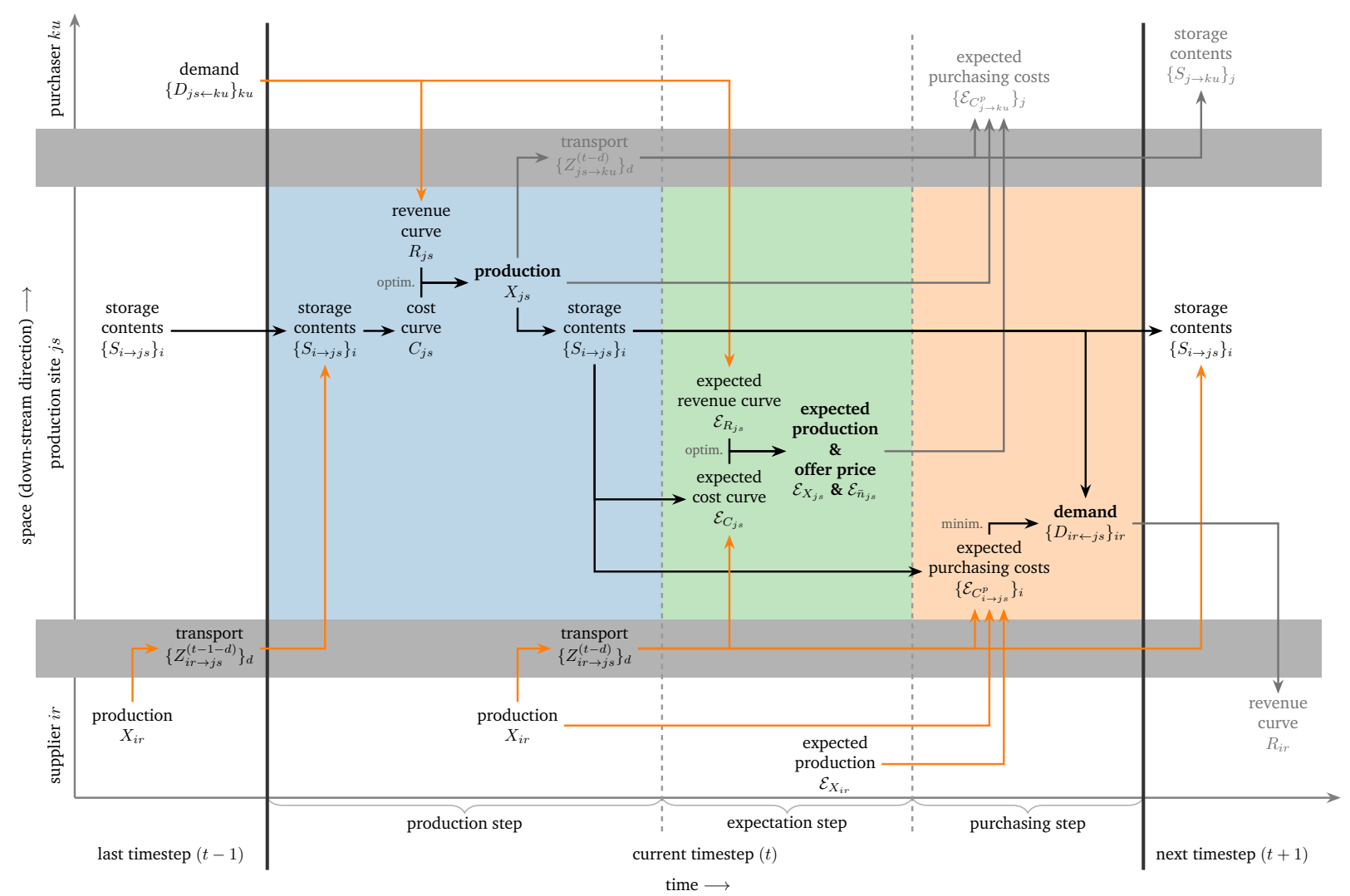

Figure A.8: Flow diagram for a firm depicting the mutual dependencies of variables within one timestep. Black arrows mark dependencies between variables of the same agents. Orange ones depict dependencies on other agent's variables or those of the connection between them. Variables and dependencies that are repeated for other agents or timesteps are grayed out.

baseline state $U_{i \rightarrow j s}^{*}$ (see Eq. (A.9)). Prices of input commodities do not depend on production level, but vary with purchasing costs.

The technology of a firm is given by the technology coefficients. These describe how many units of input commodity $i$ a firm $j s$ needs to produce one unit of output,

$$
a_{i \rightarrow j s} \equiv \frac{U_{i \rightarrow j s}}{X_{j s}} .
$$

Thus, the technology coefficients are a measure for the efficiency of a firm. Since we are interested in the short-term economic development in the first months following a disaster, no technological development is taken into account, and we assume the technology coefficients to be constant, i. e., we have $a_{i \rightarrow j s}^{(t)}=a_{i \rightarrow j s}^{*} \forall t$.

Revenue curve. The revenue curve of a firm $j$ s is constructed from the demand requests $\left\{\left(D_{j s \leftarrow k^{\prime} u^{\prime}}, n_{j s \leftarrow k^{\prime} u^{\prime}}\right)\right\}_{k^{\prime} u^{\prime}}$ it has received from its purchasers $\left\{k^{\prime} u^{\prime}\right\}_{k^{\prime} u^{\prime}}$ at the beginning of the production sub-step (cf. Figs. A.8 and Section 3.1.1). Away from equilibrium, different purchasers of $j s$ have, in general, sent different reservation prices. For bookkeeping purposes, it is thus useful to arrange the demand requests in an ordered set

$$
J_{j s} \equiv\left(\left\{k^{\prime} u^{\prime}\right\}_{k^{\prime} u^{\prime}},>\right),
$$

where the relation $>$ orders the demand requests $\left\{\left(D_{j s \leftarrow k^{\prime} u^{\prime}}, n_{j s \leftarrow k^{\prime} u^{\prime}}\right)\right\}_{k^{\prime} u^{\prime}}$ with respect to their reservation prices, i. e., $(k u)_{1}>(k u)_{2}$ means that $n_{j s \leftarrow(k u)_{1}}>n_{j s \leftarrow(k u)_{2}}$. Then, $j$ s's revenue curve may be expressed as 


$$
R_{j s}\left(\breve{X}_{j s}\right) \equiv\left\{\begin{array}{cl}
\sum_{b^{\prime} \leq l_{j s}^{\max }} v\left(D_{j s \leftarrow(k u)_{b^{\prime}}}\right) & \\
\quad+n_{j s \leftarrow(k u)_{l_{j s}^{\max }+1}}\left(\breve{X}_{j s}-\sum_{b^{\prime} \leq l_{j s}^{\max }} D_{j s \leftarrow(k u)_{b^{\prime}}}\right) & \text { for } \breve{X}_{j s} \leq D_{j s \leftarrow}, \\
R_{j s}\left(D_{j s \leftarrow}\right) & \text { for } \breve{X}_{j s}>D_{j s \leftarrow},
\end{array}\right.
$$

where the index

$$
l_{j s}^{\max } \equiv \max _{l^{\prime} \in J_{j s}}\left\{\sum_{b^{\prime} \leq l^{\prime}} D_{j s \leftarrow(k u)_{b^{\prime}}} \leq \breve{X}_{j s}\right\}
$$

denotes the largest element of the ordered set $J_{j s}$ for which the accumulated demand of the elements $b \leq l^{\max }$ is smaller than or equal to a given production level $\breve{X}_{j s}$, and

$$
D_{j s \leftarrow} \equiv \sum_{k^{\prime} u^{\prime}} D_{j s \leftarrow k^{\prime} u^{\prime}}
$$

denotes total incoming demand.

Cost curve. The production costs of a firm $j s$ consists of (i) linear commodity costs $C_{j s}^{l}$ and (ii) (other) variable production costs $C_{j s}^{v}$. Fixed costs are neglected for simplicity, which permits us to write the cost curve as

$$
\mathcal{C}_{j s}\left(\breve{X}_{j s}\right) \equiv C_{j s}^{l}\left(\breve{X}_{j s}\right)+C_{j s}^{v}\left(\breve{X}_{j s}\right) .
$$

These contributions are discussed separately in the following.

Commodity costs. Since we assume the production function to be linear with respect to commodity inputs, commodity costs are given by the sum of the values $\left\{v\left(\breve{U}_{i \rightarrow j s}\right)\right\}_{i}$ of the commodity inputs $\left\{\breve{U}_{i \rightarrow j s}\right\}_{i}$ needed for the production of $\breve{X}_{j s}$ and therefore read

$$
C_{j s}^{l}\left(\breve{X}_{j s}\right) \equiv \sum_{i^{\prime}} v\left(\breve{U}_{i^{\prime} \rightarrow j s}\right)=\sum_{i^{\prime}} v\left(a_{i^{\prime} \rightarrow j s}^{*} \breve{X}_{j s}\right)=\bar{n}_{j s}^{l} \breve{X}_{j s} .
$$

Here, we have introduced the unit commodity costs for $j s$, which are given by sum of the average unit costs of the input commodities weighted by the technology coefficients reading

$$
\bar{n}_{j s}^{l} \equiv \sum_{i^{\prime}} \bar{n}_{i^{\prime} \rightarrow j s}^{l} a_{i^{\prime} \rightarrow j s}^{*},
$$

where $\bar{n}_{i \rightarrow j s}^{l}$ denote the average unit costs for input commodity $i$ (see Eq. (A.13)). Since, in the baseline state, we have $\bar{n}_{i \rightarrow j s}^{l, *}=1 \forall i$, the baseline commodity costs equal the sum of the technology coefficients

$$
\bar{n}_{j s}^{l, *} \equiv \sum_{i^{\prime}} a_{i^{\prime} \rightarrow j s}^{*} \leq 1 .
$$

Variable production costs. Variable production costs comprise costs for labor, capital depreciation, and variable overhead. We assume marginal variable production costs to be constant up to the baseline production level and to increase linearly above. Further, we assume that the increase of marginal costs in production extension does not depend on the firm's size, but only on the ratio $\breve{X}_{j s} / \lambda_{j s} X_{j s}^{*}$ of its current production level $\breve{X}_{j s}$ with respect to the (forced) baseline production level $\lambda_{j s} X_{j s}^{*}$. This assumption is important for demand redistribution, because it guarantees that suppliers are driven uniformly into production extension. Assuming variable production costs to be at least one time continuously differentiable then permits to write them as 


$$
C_{j s}^{v}\left(\breve{X}_{j s}\right) \equiv \begin{cases}n_{j s}^{v} \breve{X}_{j s} & \text { for } \breve{X}_{j s} \in\left[0, \lambda_{j s} X_{j s}^{*}\right], \\ n_{j s}^{v} \breve{X}_{j s}+\frac{\Delta n_{j}^{\text {in }, v,>}}{\lambda_{j s} X_{j s}^{*}}\left(\breve{X}_{j s}-\lambda_{j s} X_{j s}^{*}\right)^{2} & \text { for } \left.\left.\breve{X}_{j s} \in\right] \lambda_{j s} X_{j s}^{*}, \lambda_{j s} \beta_{j} X_{j s}^{*}\right],\end{cases}
$$

where $n_{j s}^{v}$ denotes the unit variable production costs below production extension, and $\Delta n_{j}^{\mathrm{in}, v,>}$ is the coefficient for the cost increase in production extension. While the former may vary from firm to firm the later is assumed to vary among sectors only.

Whereas commodity costs in the baseline state can be directly derived from the flows comprised in the MRIO-tables, variable production costs usually cannot. This is the reason why we calculate these costs from the value added in the baseline state. The latter may be written as the difference of revenue and commodity costs, on the one hand, and as the sum of variable production costs and profit, on the other hand,

$$
\operatorname{VA}_{j s}\left(\breve{X}_{j s}\right) \equiv R_{j s}\left(\breve{X}_{j s}\right)-C_{j s}^{l}\left(\breve{X}_{j s}\right)=C_{j s}^{v}\left(\breve{X}_{j s}\right)+\Pi_{j s}\left(\breve{X}_{j s}\right) .
$$

By inserting Eqs. (A.18), (A.21), and (A.22) in the above equation and dividing by the production level $\breve{X}_{j s}$, we obtain an expression for the value added per unit produced

$$
\frac{\mathrm{VA}_{j s}\left(\breve{X}_{j s}\right)}{\breve{X}_{j s}}=\bar{n}_{j s}-\bar{n}_{j s}^{l}=n_{j s}^{v}+\pi_{j s}
$$

where $\pi_{j s}$ denotes the firm's monopolistic markup. In the baseline state, the average unit price equals unity $\bar{n}_{j s}^{*}=1$, and, thus, Eq. (A.24) simplifies to

$$
\frac{\mathrm{VA}_{j s}^{*}}{X_{j s}^{*}}=1-\bar{n}_{j s}^{l, *}=n_{j s}^{v, *}+\pi_{j s}^{*},
$$

where $\mathrm{VA}_{j s}^{*} \equiv \operatorname{VA}_{j s}\left(X_{j s}^{*}\right), n_{j s}^{v, *} \geq 0$, and $\pi_{j s}^{*} \geq 0$ denote the baseline values of value added, variable production costs per unit produced, and monopolistic markup, respectively. Note, that since $n_{j s}^{v, *}$ and $\pi_{j s}^{*}$ are both non-negative, the right-hand-side of the last equality in Eq. (A.25) is always positive. This implies that only firms with positive baseline value added are considered, and, for instance, heavily subsidized sectors with negative value added are removed from the network. However, in practice this constraint affects only very few firms.

Next, we discuss how to determine the variable production $\operatorname{costs} n_{j s}^{v}$. For that, we first employ Eq. (A.25) to calculate the value added per unit produced in the baseline state $\mathrm{VA}_{j s}^{*} / X_{j s}^{*}$ from the MRIO-tables, which determines the value of $n_{j s}^{v, *}+\pi_{j s}^{*}$. Setting $\pi_{j s}^{*}$ exogenously as detailed below and additionally assuming that $n_{j s}^{v}$ does not change in disequilibrium permits to write the latter as

$$
n_{j s}^{v}=n_{j s}^{v * *} \equiv \frac{\mathrm{VA}_{j s}^{*}}{X_{j s}^{*}}-\pi_{j s}^{*}
$$

To calculate $\pi_{j s}^{*}$, we introduce the monopolistic markup in the baseline state $\pi_{j}^{*}$ as an exogenous parameter that may differ among sectors. Depending on the value of $\pi_{j}^{*}$, this monopolistic markup may not be achievable for all firms of sector $j$, because for the less efficient ones the difference of baseline product price, $\bar{n}_{j}^{*}=1$, and unit commodity costs $\bar{n}_{j s}^{l, *}$ may be smaller than $\pi_{j}^{*}$. Therefore, we see from Eq. (A.25) that

$$
\pi_{j s}^{*} \equiv \min \left[\pi_{j}^{*}, 1-\bar{n}_{j s}^{l, *}\right]
$$

is a meaningful definition of the baseline monopolistic markup that guarantees $\pi_{j s}^{*}$ to be positive. Note that away from the baseline state, the profit realized, and, thus, the monopolistic markup depend on prices. In consequence, $\pi_{j s}$ can differ from its baseline value $\pi_{j s}^{*}$.

With the above assumption for commodity and variable production costs, we obtain the following cost curve 


$$
C_{j s}\left(\breve{X}_{j s}\right) \equiv \begin{cases}n_{j s}^{c} \breve{X}_{j s} & \text { for } \breve{X}_{j s} \in\left[0, \lambda_{j s} X_{j s}^{*}\right], \\ n_{j s}^{c} \breve{X}_{j s}+\frac{\Delta n_{j}^{\text {in }, v,>}}{\lambda_{j s} X_{j s}^{*}}\left(\breve{X}_{j s}-\lambda_{j s} X_{j s}^{*}\right)^{2} & \text { for } \left.\left.\breve{X}_{j s} \in\right] \lambda_{j s} X_{j s}^{*}, \lambda_{j s} \beta_{j} X_{j s}^{*}\right],\end{cases}
$$

which is depicted by blue solid lines in the lower panels of Fig. 2. Below production extension, it increases linearly with production level $\breve{X}_{j s}$, and its slope is given by the unit production costs

$$
n_{j s}^{c} \equiv \bar{n}_{j s}^{l}+n_{j s}^{v} \text {. }
$$

However, in production extension, the slope of the cost curve increases smoothly due to a linear increase in marginal variable production costs. More precisely, by taking the derivative of Eq. (A.28) with respect to $\breve{X}_{j s}$, denoted by $(\cdot)^{\prime}$, we obtain the marginal cost curve as

$$
C_{j s}^{\prime}\left(\breve{X}_{j s}\right) \equiv \begin{cases}n_{j s}^{c} & \text { for } \breve{X}_{j s} \in\left[0, \lambda_{j s} X_{j s}^{*}\right], \\ n_{j s}^{c}+\frac{2 \Delta n_{j}^{\mathrm{in}, v,>}}{\lambda_{j s} X_{j s}^{*}}\left(\breve{X}_{j s}-\lambda_{j s} X_{j s}^{*}\right) & \text { for } \left.\left.\breve{X}_{j s} \in\right] \lambda_{j s} X_{j s}^{*}, \lambda_{j s} \beta_{j} X_{j s}^{*}\right] .\end{cases}
$$

For subsequent calculations, we eventually define the extra variable production costs arising in production extensions as

$$
\Delta C^{v,>}\left(\breve{X}_{j s}\right) \equiv \begin{cases}0 & \text { for } \breve{X}_{j s} \in\left[0, \lambda_{j s} X_{j s}^{*}\right] \\ \frac{\Delta n_{j}^{\mathrm{in}, v,>}}{\lambda_{j s} X_{j s}^{*}}\left(\breve{X}_{j s}-\lambda_{j s} X_{j s}^{*}\right)^{2} & \text { for } \left.\left.\breve{X}_{j s} \in\right] \lambda_{j s} X_{j s}^{*}, \lambda_{j s} \beta_{j} X_{j s}^{*}\right] .\end{cases}
$$

Profit maximizing production level. In the production step, each firm $j s$ determines its actual production $X_{j s}$ by maximizing its profit

$$
\Pi_{j s}\left(\breve{X}_{j s}\right) \equiv R_{j s}\left(\breve{X}_{j s}\right)-C_{j s}\left(\breve{X}_{j s}\right)
$$

under the constraint that production must not exceed productive capacity $\hat{X}_{j s}$, which reads

$$
X_{j s} \equiv \underset{\breve{X}_{j s}}{\operatorname{argmax}}\left[\Pi_{j s}\left(\breve{X}_{j s}\right)\right] \text { subject to } 0 \leq \breve{X}_{j s} \leq \hat{X}_{j s} .
$$

The simple forms of revenue and costs curves permit to determine $X_{j s}$ analytically. For this, $j s$ first determines its profit maximizing production level $X_{j s}^{\text {opt }}$ without taking its productive capacity into account. It follows from Eq. (A.32) that the first-order condition for a production level to be profit maximizing is that marginal revenue equals marginal costs. Further, we see from the definitions of revenue curve $R_{j s}$ in Eq. (A.18) and marginal cost curve $C_{j s}^{\prime}$ in Eq. (A.30) that, below production extension $\left(\breve{X}_{j s} \leq \lambda_{j s} X_{j s}^{*}\right)$, the profit maximizing production level is reached, when all purchasers are served that have bid reservation prices at least equal to $j s$ 's unit production costs $n_{j s}^{c}$ (see Eq. (A.29)). In the following, this subset of the order set of purchasers $J_{j s}$ (see Eq. (A.17)) is denoted by

$$
J_{j s}^{\mathrm{opt}} \equiv\left\{l^{\prime} \in J_{j s} \mid n_{j s \leftarrow(k u)_{l^{\prime}}} \leq n_{j s}^{c}\right\} \subseteq J_{j s} .
$$

In production extension, i.e., $\left.\breve{X}_{j s} \in\right] \lambda_{j s} X_{j s}^{*}, \lambda_{j s} \beta_{j s} X_{j s}^{*}$, the super-linear increase of variable production costs (cf. Eq. (A.22)) renders the shape of the cost curve $C_{j s}$ more complex. However, since $C_{j s}$ remains concave $\left(C_{j s}^{\prime \prime}\left(X_{j s}\right) \geq 0\right)$, and $R_{j s}$ is convex $\left(R_{j s}^{\prime \prime}\left(X_{j s}\right) \leq 0\right.$ ), we may still obtain $X_{j s}^{\text {opt }}$ by equating marginal revenue and marginal costs yielding 


$$
\begin{array}{rlrl}
R_{j s}^{\prime}\left(X_{j s}^{\mathrm{opt}}\right) & =C_{j s}^{\prime}\left(X_{j s}^{\mathrm{opt}}\right) \\
\Leftrightarrow & n_{j s \leftarrow}^{\mathrm{opt}} & =n_{j s}^{c}+\frac{2 \Delta n_{j}^{\mathrm{in}, v,>}}{\lambda_{j s} X_{j s}^{*}}\left(X_{j s}^{\mathrm{opt}}-\lambda_{j s} X_{j s}^{*}\right) \\
\Leftrightarrow & X_{j s}^{\mathrm{opt}} & =\lambda_{j} X_{j s}^{*}\left[1+\frac{n_{j s \leftarrow}^{\mathrm{opt}}-n_{j s}^{c}}{2 \Delta n_{j}^{\mathrm{in}, v,>}}\right],
\end{array}
$$

where $n_{j s \leftarrow}^{\mathrm{opt}}$ denotes the price of the lowest priced purchaser that would obtain a non-zero share of $X_{j s}^{\mathrm{opt}}$. Concluding, the optimal production level is given by

$$
X_{j s}^{\mathrm{opt}} \equiv \begin{cases}\sum_{l^{\prime} \in J_{j s}^{\mathrm{opt}}} D_{j s \leftarrow(k u)_{l^{\prime}}} & \text { for } X_{j s}^{\mathrm{opt}} \leq \lambda_{j s} X_{j s}^{*}, \\ \lambda_{j} X_{j s}^{*}\left[1+\frac{n_{j s}^{\mathrm{opt}}-n_{j s}^{\mathrm{c}}}{2 \Delta n_{j}^{\mathrm{in}, v,>}}\right] & \text { for } X_{j s}^{\mathrm{opt}}>\lambda_{j s} X_{j s}^{*} .\end{cases}
$$

To determine its actual production level

$$
X_{j s} \equiv \min \left[X_{j s}^{\mathrm{opt}}, \hat{X}_{j s}\right],
$$

$j s$ caps $X_{j s}^{\text {opt }}$ with its productive capacity $\hat{X}_{j s}$ (see Eq. (A.15)). For the production $j s$ uses, as determined by its technology, an amount of input commodity $i$ of

$$
U_{i \rightarrow j s}=a_{i \rightarrow j s}^{*} X_{j s}
$$

After production, firms distribute their output among those purchasers with sufficiently high reservation prices, starting with the highest-bidding purchaser,

$$
Z_{i r \rightarrow j s}= \begin{cases}0 & \text { for } n_{i r \leftarrow j s}<n_{i r \leftarrow(k u)_{l_{i r}^{\max }}} \text { (see Eq. (A.19)), } \\ D_{i r \leftarrow j s} & \text { for } n_{i r \leftarrow j s}>n_{i r \leftarrow(k u)_{l_{i r}}} \text { (see Eq. (A.19)), } \\ X_{i r}-\sum_{b^{\prime} \leq l_{i r}^{\max }} D_{i r \leftarrow(k u)_{b^{\prime}}} & \text { otherwise (see Eq. (A.18)). }\end{cases}
$$

Note that the reservation prices of its purchasers determine firm's $j s$ average production price, i.e., its selling price

$$
\bar{n}_{j s} \equiv \frac{R_{j s}\left(X_{j s}\right)}{X_{j s}} .
$$

Since in disequilibrium it can happen that not all purchasers are served, $\bar{n}_{j s}$ does not necessarily equal the average reservation price of the purchasers

$$
\bar{n}_{j s}^{p} \equiv \frac{R_{j s}\left(D_{j s \leftarrow)}\right)}{D_{j s \leftarrow}}
$$

\section{Appendix A.2.2. Expectation step}

In the expectation step, each firm $j s$ determines the production level $\mathcal{E}_{X_{j s}}$ it expects to be profit-maximizing in the next timestep as well as the corresponding offer price $\mathcal{E}_{\bar{n}_{j s}}$, i. e., the average price to which it expects to be able to sell its product in the next timestep. Note that we use the notation $\mathcal{E}_{(\cdot)}$ to describe the expectation an agent forms at time $(t)$ on the value of its own property $(\cdot)$ in the next timestep $(t+1)$. First, $j s$ has to form expectations on its revenue and cost curves in the next timestep. Then, $j s$ can determine the production level that it expects to be profit-maximizing. 
Expected revenue curve. To derive its expected revenue curve, $j s$ has to make assumptions on exogenous forcing and incoming demand in the next timestep. For that, it assumes that

(i) the exogenous forcing $\lambda_{j s}$ remains at its current level, and that,

(ii) the structure of incoming demand requests, with respect to demanded quantities and reservation prices, remains unchanged.

Assumption (i) expresses that arrival and exact duration of extreme events are considered to be unpredictable. Assumption (ii) accounts for the very limited network overview of the agents.

Firm's $j s$ offer price $E_{\bar{n}_{j s}}$ is calculated analogously to the average selling price $\bar{n}_{j s}$ defined in Eq. (A.38) and, therefore, reads

$$
\mathcal{E}_{\bar{n}_{j s}} \equiv \frac{\mathcal{E}_{R_{j s}}\left(\mathcal{E}_{X_{j s}}\right)}{\mathcal{E}_{X_{j s}}}
$$

Further, it is worthy to note that, according to assumption (ii), $\mathcal{E}_{R_{j s}}$ is simply identical to $R_{j s}$ (cf. Eq. (A.18)).

Expected cost curve. To obtain the cost curve $\mathcal{E}_{C_{j s}}$ firm $j s$ expects to have in the next timestep, it firstly has to determine the unit costs $\mathcal{E}_{n_{j s}^{c}}$ it expects to have. This is done analogously to the calculation of $n_{j s}^{c}$ in Eq. (A.29). Note that, nevertheless, $\mathcal{E}_{n_{j s}^{c}}$ can differ from $n_{j s}^{c}$ due to the input flows $j s$ received in the current timestep as well as changes in $j$ s's input inventory levels. Eventually, $\mathcal{E}_{C_{j s}}$ is obtained from Eq. (A.28) by substituting $n_{j s}^{c}$ with $\mathcal{E}_{n_{j s}^{c}}$.

Maximization of expected profit. Analogously to profit $\Pi_{j s}$ (see Eq. (A.32)), the expected profit of a firm $j s$ is defined as the difference of expected revenue and cost curves reading

$$
\mathcal{E}_{\Pi_{j s}}\left(\mathcal{E}_{\breve{X}_{j s}}\right) \equiv \mathcal{E}_{R_{j s}}\left(\mathcal{E}_{\breve{X}_{j s}}\right)-\mathcal{E}_{C_{j s}}\left(\mathcal{E}_{\breve{X}_{j s}}\right) .
$$

Before $j s$ can determine the production level $\mathcal{E}_{X_{j s}}$ it expects to be profit-maximizing in the next timestep, $j s$ first has to estimate its productive capacity $\mathcal{E}_{\hat{X}_{j s}}$ for the next timestep. For this, we first note that, at the end of the production step, $j s$ has received the input commodities it can use for production in the next timestep. Knowing input flow and storage content, $j s$ can then calculate the quantity $\mathcal{E}_{\hat{U}_{i \rightarrow j s}}$ of each input commodity $i$ that it expects to use. This is done analogously to the calculation of $\hat{U}_{i \rightarrow j s}$ in Eq. (A.14). Next, $j s$ determines its expected productive capacity $\mathcal{E}_{\hat{X}_{j s}}$ by evaluating whether $\mathcal{E}_{\hat{X}_{j s}}$ is limited by the input commodity with the lowest possible use or by the expected external forcing on the productive capacity (cf. Eq. (A.15)) reading

$$
\mathcal{E}_{\hat{X}_{j s}} \equiv \min \left[\min _{i^{\prime}}\left[\frac{\mathcal{E}_{\hat{U}_{i^{\prime} \rightarrow j s}}}{\hat{U}_{i^{\prime} \rightarrow j s}^{*}}\right], \lambda_{j s} \beta_{j}\right] X_{j s}^{*} .
$$

The expected production level $\mathcal{E}_{X_{j s}}$ may be determined, analogously to the current production level $X_{j s}$ (see Eq. (A.33)), by a constrained maximization of expected profit, which reads

$$
\mathcal{E}_{X_{j s}} \equiv \underset{\mathcal{E}_{\breve{X}_{j s}}}{\operatorname{argmax}}\left[\mathcal{E}_{\Pi_{j s}}\left(\mathcal{E}_{\breve{X}_{j s}}\right)\right] \text { subject to } 0 \leq \mathcal{E}_{\breve{X}_{j s}} \leq \mathcal{E}_{\hat{X}_{j s}} \text { and } \pi_{j s}^{*}-\frac{\mathcal{E}_{\Pi_{j s}}}{\mathcal{E}_{X_{j s}}} \leq 0 .
$$

Comparing Eq. (A.43) to the constrained profit maximization of Eq. (A.33), we note one structural difference: in the optimization problem of Eq. (A.43) it is implied that $j s$ 's expected monopolistic markup $\mathcal{E}_{\Pi_{j s}} / \mathcal{E}_{X_{j s}}$ has to be at least equal to its markup in the baseline state $\pi_{j s}^{*}$ ( $2^{\text {nd }}$ constraint in Eq. (A.43)), which is assumed to be the target markup. This additional constraint prevents $j s$ from communicating low offer prices, which would entail demand requests with reservation prices too low to permit $j s$ keeping up a margin of $\pi_{j s}^{*}$.

The offer price $\mathcal{E}_{\bar{n}_{j s}}$ may then be calculated according to Eq. (A.40). Eventually, each firm $j s$ communicates $\mathcal{E}_{X_{j s}}$ and $\mathcal{E}_{\bar{n}_{j s}}$ to its purchasers $\{i r\}_{i, r}$. These parameters will enable $j s$ 's purchasers to form expectations on the shape of $j s$ 's supply curve in the next timestep as discussed in Appendix A.2.3. 
A special case arises, when $j s$ has not received any demand, i. e., $D_{j s \leftarrow}=0$. Then $j s$ cannot estimate an expected revenue curve. In consequence, $j s$ is not able to employ Eqs. (A.40) and (A.43) to determine $\mathcal{E}_{\bar{n}_{j s}}$ and $\mathcal{E}_{X_{j s}}$, respectively. In this case, we assume that $j s$ communicates its expected production costs as offer price $\mathcal{E}_{\bar{n}_{j s}}=\mathcal{E}_{n_{j s}^{c}}$, and the minimum of possible and forced baseline production level as expected production level $\mathcal{E}_{X_{j s}}=\min \left[\lambda_{j s} X_{j s}^{*}, \hat{X}_{j s}\right]$.

\section{Appendix A.2.3. Purchasing step}

In the purchasing step, firms decide on their distribution of demand requests (with respect to quantities and reservation prices) among their purchasers by minimizing their expected purchasing costs. First, we discuss the firms' outgoing demand. Next, we detail how firms form expectations on their suppliers' supply curves. Then, we consider the additional costs for transportation arising in non-equilibrium situations, before eventually discussing the cost minimization.

Outgoing demand. The cumulative outgoing demand of firm $j s$ for commodity $i$ reads

$$
D_{i \leftarrow j s} \equiv \min \left[\mathcal{E}_{U_{i \rightarrow j s}}+\frac{\Delta S_{i \rightarrow j s}}{\tau_{i \rightarrow j s}}, \mathcal{E}_{D_{i \leftarrow j s}^{j s}}^{\text {max }}\right] .
$$

Here, $\mathcal{E}_{U_{i \rightarrow j}}$ denotes the amount of commodity $i$ that $j s$ expects to use in the next timestep. It is derived from $j s$ 's expected profit-maximizing production level (see Eq. (A.42)). The demand changes if the inventory level for commodity $i$ deviates from its baseline value $S_{i \rightarrow j s}^{*}$. This is described by the storage deviation

$$
\Delta S_{i \rightarrow j s} \equiv S_{i \rightarrow j s}^{*}-S_{i \rightarrow j s}+T_{i \rightarrow j s}^{\text {def }}
$$

which also accounts for a deviation

$$
T_{i \rightarrow j s}^{\operatorname{def}} \equiv T_{i \rightarrow j s}-T_{i \rightarrow j s}^{*} .
$$

of the rolling inventory $T_{i \rightarrow j s}$ (see. Eq. (A.7)) from its baseline value $T_{i \rightarrow j s}^{*}$. In times of scarcity $\left(\Delta S_{i \rightarrow j s}>0\right)$ or abundance $\left(\Delta S_{i \rightarrow j s}<0\right)$, js increases or decreases its demand, respectively. The timescale at which $j s$ aims to balance storage anomalies is given by $\tau_{i \rightarrow j s}$. Further, the minimum condition in Eq. (A.44) expresses that demand is limited by the maximal demand $j s$ expects to be able to source from its suppliers $\mathcal{E}_{D_{i \leftarrow j s}^{j s}}^{j \max } \equiv \sum_{r^{\prime}} \mathcal{E}_{D_{i r^{\prime} \leftarrow j s}^{j s}}^{\max }$ in the next timestep ${ }^{17}$. The latter is the sum of the productive capacities $\left\{\mathcal{E}_{D_{i r^{\prime} \leftarrow j s}^{j s}}^{\max }\right\}_{r^{\prime}}$ that $j s$ expects its suppliers to have in the next timestep (see next section).

Estimates on suppliers' supply curves. To estimate its purchasing costs, each firm $j$ s has to form expectations on its suppliers' supply curves $\left\{\mathcal{E}_{\tilde{n}_{i^{\prime} r^{\prime}}}^{j s}\right\}_{i^{\prime} r^{\prime}}$ in the next timestep. To obtain $\mathcal{E}_{\tilde{n}_{i r}}^{j s}$ of a supplier $i r, j s$ may refer to $i r$ 's delivery in the production step as well as the expected upcoming production level $\mathcal{E}_{X_{i r}}$ and the offer price $\mathcal{E}_{\bar{n}_{i r}}$ that $i r$ has communicated in the expectation step (cf. Appendix A.2.2). However, $j s$ is lacking information on the demand requests of its purchasing competitors. For a sound estimation of those, $j s$ would need, for instance, information on the importance of the common supplier $i r$ for each of $j s$ 's competitors. This would require, on the one hand, that $j s$ has information on the rest of their business connections, i. e., on the network topology. On the other hand, $j s$ would need information on its competitors' current market situations, e. g., if they suffer from other supply shortages. Unfortunately, due to its limited network oversight, $j s$ has too little information for such kinds of assessment. In consequence, $j s$ has to make educated guesses on its competitors' demand requests regarding quantities and prices. With respect to the quantities, $j s$ assumes that

\footnotetext{
${ }^{17}$ Here, the notation $\mathcal{E}_{(\cdot)}^{(\cdot)}$ denotes the expectation that an agent - indicated by the upper index - makes in timestep $(t)$ on the value of another agent's property in timestep $(t+1)$ - indicated by the lower index. For instance, $\mathcal{E}_{\breve{n}_{i r}}^{j s}$ denotes the expectation that $j s$ has at time $t$ on $i r$ 's supply curve in the next timestep $(t+1)$.
} 
(i) its purchasing competitors keep their demand distributions fixed, i. e., from the common supplier ir they demand the same share of its expected production in the next timestep $\mathcal{E}_{X_{i r}}$ as they expect to have received from its current production $X_{i r}$.

Furthermore, $j s$ forms expectations on $i r$ 's production level in the current timestep $X_{i r}$, $i r$ 's production level in the baseline state $X_{i r}^{*}$, ir's forcing level in the next timestep $\lambda_{i r}^{(t+1)}$, and sector $i$ 's production extension factor $\beta_{i}$. These expectations are denoted by $\mathcal{E}_{X_{i r}^{(t)}}^{j s}, \mathcal{E}_{X_{i r}^{*}}^{j s}, \mathcal{E}_{\lambda_{i r}}^{j s}$, and $\mathcal{E}_{\beta_{i}}^{j s}$, respectively. As shown below, they permit $j s$ to form an expectation $\mathcal{E}_{\breve{X}_{i r}}^{j s}=\mathcal{E}_{\breve{X}_{i r}}^{j s}\left(\breve{D}_{i r \leftarrow j s}\right)$ on $i r$ 's production in the next timestep in terms of the demand $\breve{D}_{i r \leftarrow j s}$ that $j s$ addresses to $i r$. In addition, they enable $j s$ to obtain the minimum demand that would drive $i r$ into production extension as well as the maximum demand it can expect to be fulfilled by $i r$. To keep the model simple, we assume that $j s$ 's expectation on the above quantities are as straightforward as possible, i. e., we assume that $j s$ knows $X_{i r}^{(t)}, X_{i r}^{*}$, and $\beta_{i}$ exactly:

$$
\mathcal{E}_{X_{i r}^{(t)}}^{j s}=X_{i r}^{(t)}, \quad \quad \mathcal{E}_{X_{i r}^{*}}^{j s}=X_{i r}^{*}, \quad \text { and } \quad \mathcal{E}_{\beta_{i}}^{j s}=\beta_{i} .
$$

Further, we assume that $j s$ has the same expectation on the forcing $\lambda_{i r}^{(t+1)}$ its supplier $i r$ will perceive in the next timestep as ir has itself. This can be written as

$$
\mathcal{E}_{\lambda_{i r}}^{j s}=\mathcal{E}_{\lambda_{i r}}=\lambda_{i r},
$$

where we have employed the assumption that ir expects the forcing to remain at its current level (see Appendix A.2.2).

Next, we may conclude from assumption (i) that $\mathcal{E}_{\tilde{X}_{i r}}^{j s}$ can be written as

$$
\begin{aligned}
\mathcal{E}_{\breve{X}_{i r}}^{j s}\left(\breve{D}_{i r \leftarrow j s}\right) & =\breve{D}_{i r \leftarrow j s}+\mathcal{E}_{X_{i r}} \frac{X_{i r}-Z_{i r \rightarrow j s}}{X_{i r}} \\
& =\left(1+\mathcal{E}_{\breve{s}_{i r \leftarrow j s} s}\left(\breve{D}_{i r \leftarrow j s}\right)-s_{i r \leftarrow j s}\right) \mathcal{E}_{X_{i r}} .
\end{aligned}
$$

This can be seen as follows: the second term on the right-hand-side of Eq. (A.47a) describes the share $j s$ 's competitors have received from $i r$ 's current production. According to assumption (i), this is also the share $j s$ expects those to obtain from $\mathcal{E}_{X_{i r}}$. In Eq. (A.47b), $\mathcal{E}_{\breve{X}_{i r}}^{j s}$ has been rewritten as a function of the share

$$
\mathcal{E}_{\breve{s}_{i r \leftarrow j s}}^{j s}\left(\breve{D}_{i r \leftarrow j s}\right) \equiv \frac{\breve{D}_{i r \leftarrow j s}}{\mathcal{E}_{X_{i r}}}
$$

that $j s$ expects to obtain from $i r$ 's next production if it demands the quantity $\breve{D}_{i r \leftarrow j s} ; s_{i r \leftarrow j s} \equiv Z_{i r \leftarrow j s} / X_{i r}$ denotes $j s$ 's share of $i r$ 's current production.

From Eq. (A.47b), we may note two important findings. First, $\mathcal{E}_{\tilde{X}_{i r}}^{j s}=\mathcal{E}_{\tilde{X}_{i r}}^{j s}\left(\mathcal{E}_{\tilde{s}_{i r \leftarrow j s}}^{j s}\right)$ may be expressed as a function of $j s$ 's expected share $\mathcal{E}_{\widetilde{s}_{i r \leftarrow j s}}^{j s}$. This is helpful to argue that also $j s^{\prime}$ 's expectation on $i r$ 's upcoming demand curve $\mathcal{E}_{\tilde{n}_{i r}}^{j s}=\mathcal{E}_{\tilde{n}_{i r}}^{j s}\left(\mathcal{E}_{\widetilde{s}_{i r \leftarrow j s}}^{j s}\right)$ depends only upon $\mathcal{E}_{\breve{s}_{i r \leftarrow j s}}^{j s}$. Second, if $j s$ 's share remains unchanged $\left(\mathcal{E}_{\bar{s}_{i r \leftarrow j s}}^{j s}=s_{i r \leftarrow j s}\right)$, the expectation $j s$ has on $i r$ 's upcoming production level equals $i r$ 's own expectation, i. e., we have

$$
\mathcal{E}_{\tilde{X}_{i r}}^{j s}\left(s_{i r \leftarrow j s}\right)=\mathcal{E}_{X_{i r}} .
$$

By inserting $\mathcal{E}_{\breve{X}_{i r}}^{j s}=\lambda_{i r} \beta_{i} X_{i r}^{*}$ into Eq. (A.47a), the maximum demand request $\mathcal{E}_{D_{i r \leftarrow j s}^{\max }}^{\max _{j}}$ that $j s$ expects to be fulfilled by $i r$ reads 


$$
\mathcal{E}_{D_{i r \leftarrow j s}^{j s}}^{j s a x} \lambda_{i r} \beta_{i} X_{i r}^{*}-\mathcal{E}_{X_{i r}} \frac{X_{i r}-Z_{i r \rightarrow j s}}{X_{i r}} .
$$

From Eq. (A.47b) then follows that $\mathcal{E}_{D_{i r \leftarrow j s}^{j s}}^{\text {max }}$ corresponds to a maximum share of

$$
\mathcal{E}_{s_{i r \leftarrow j s}^{j s}}^{j \max } \equiv s_{i r \leftarrow j s}-1+\frac{\lambda_{i r} \beta_{i} X_{i r}^{*}}{\mathcal{E}_{X_{i r}}} .
$$

Similarly, by inserting $\mathcal{E}_{\tilde{X}_{i r}}^{j s}=\lambda_{i r} X_{i r}^{*}$ into Eq. (A.47b), it follows directly that the minimum share that $j s$ expects to drive supplier $i r$ into production extension, is given by

$$
\mathcal{E}_{s_{i r \leftarrow j s}^{<}}^{j s} \equiv \max \left[0, s_{i r \leftarrow j s}-1+\frac{\lambda_{i r} X_{i r}^{*}}{\mathcal{E}_{X_{i r}}}\right] .
$$

Next, we derive the supply curve $\mathcal{E}_{\tilde{n}_{i r}}^{j s}$ that $j s$ expects $i r$ to have in the next timestep. Since $j s$ has no information on the reservation prices of its purchasing competitors it has to make two additional assumptions. Firstly, $j s$ assumes that

(ii) by bidding the offer price $\mathcal{E}_{\bar{n}_{i r}}$ communicated by supplier $i r$, it will receive the same share of $i r$ 's production as in the current timestep.

Note that this is a meaningful strategy, since according to assumption (i) its purchasing competitors keep their shares fixed. If additionally they have the same strategy as $j s$ to determine their reservation price, they offer $\mathcal{E}_{\bar{n}_{i r}}$, too, and $j s$ 's demand request will be successfully fulfilled. Secondly, $j s$ assumes that

(iii) $i r$ 's supply curve for the next timestep is based on its production costs, which is a reasonable assumption if the market is competitive. This implies that if $j s$ aims to increase its share beyond $s_{i r \leftarrow j s}$, supplier $i r$ would have to extend its production. In consequence, $j s$ would have to compensate $i r$ for potential additional expenses such as long hours of workers. In reverse, for $\mathcal{E}_{s_{i r \leftarrow j s}}^{j s}<\mathcal{E}_{s_{i r \leftarrow j s}^{<}}^{j s}$, it expects that supplier $i r$ will be willing to fulfill its demand $\breve{D}_{i r \leftarrow j s}$ to a price lower than $\mathcal{E}_{\bar{n}_{i r}}$.

Here, we assume that $\mathcal{E}_{\tilde{n}_{i r}}^{j s}$ increases linearly starting from the unit production costs $\mathcal{E}_{n_{i r}^{c}}^{j s}$ that $j s$ expects supplier $i r$ to have in the next timestep, i. e., $\mathcal{E}_{\tilde{n}_{i r}}^{j s}(0)=\mathcal{E}_{n_{i r}^{c}}^{j s}$, up to the unit costs $\mathcal{E}_{n_{i r}^{<}}^{j s} \equiv \mathcal{E}_{\tilde{n}_{i r}}^{j s}\left(\mathcal{E}_{s_{i r}^{<}<s}^{j s}\right)$ that $j s$ expects $i r$ to have if it demands the share $\mathcal{E}_{s_{i r \leftarrow j s}^{<}}^{j s}$.

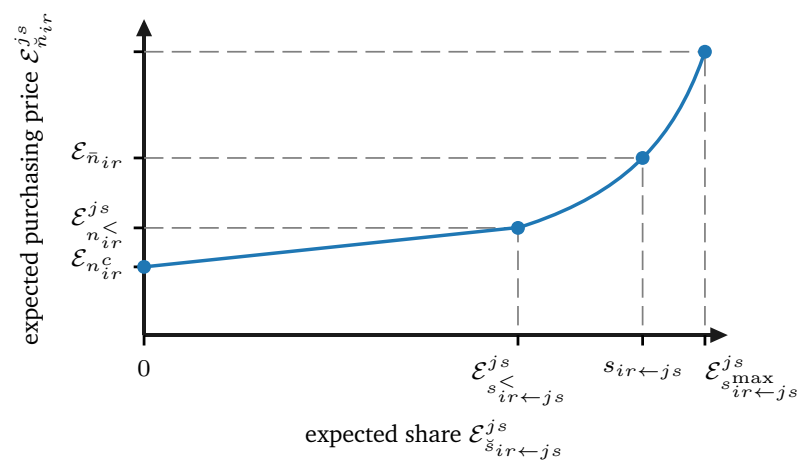

Figure A.9: Sketch of the supply curve $\mathcal{E}_{\breve{n}_{i r}}^{j s}$ that economic agent $j s$ expects its supplier $i r$ to have in the next timestep.

The resulting curve $\mathcal{E}_{\tilde{n}_{i r}}^{j s}=\mathcal{E}_{\tilde{n}_{i r}}^{j s}\left(\mathcal{E}_{\tilde{s}_{i r \leftarrow j s}}^{j s}\right)$ is depicted in Fig. A.9. In production extension $\left(\mathcal{E}_{\tilde{s}_{i r \leftarrow j s}}^{j s}>\mathcal{E}_{s_{i r \leftarrow j s}^{<}}^{j s}\right)$, it has the same shape as supplier $i r$ 's cost curve (cf. Eq. (A.28)) with $\mathcal{E}_{n_{i r}^{<}}^{j s}$ taking the role of $n_{i r}^{c}$. From Eq. (A.28) 
for the cost curve, we see that, to estimate the shape of $i r$ 's cost curve, $j s$ needs to form expectations on sector $i$ 's price increase in production extension $\Delta n_{i}^{\mathrm{in}, v,>}$, and $i r$ 's unit production costs below production extension in the next timestep $n_{i r}^{c,(t+1)}$. These expectations are denoted by $\mathcal{E}_{\Delta n_{i}^{\text {in }, v,>}}^{j s}$, and $\mathcal{E}_{n_{i r}^{c}}^{j s}$, respectively. For simplicity, we assume that $j s$ expectations on $\Delta n_{i}^{\mathrm{in}, v,>}$ are perfect, i. e., $\mathcal{E}_{\Delta n_{i}^{\mathrm{in}, v,>}}=\Delta n_{i}^{\mathrm{in}, v,>}$, and that $j s$ has the same expectations on $n_{i r}^{c,(t+1)}$ as $i r$ itself, i. e., $\mathcal{E}_{n_{i r}^{j}}^{j s}=\mathcal{E}_{n_{i r}^{c}}$.

Next, we derive an expression for $\mathcal{E}_{n_{i r}^{<}}^{j s}$. For that, we first note from the expression for $i r$ 's cost curve (see Eq. (A.28)) that assumption (iii) permits us to write $j s$ 's expectation on $i r$ 's revenue in the next timestep as

$$
\begin{aligned}
& \mathcal{E}_{\tilde{n}_{i r}}^{j s}\left(\mathcal{E}_{\tilde{X}_{i r}}^{j s}\right) \mathcal{E}_{\tilde{X}_{i r}}^{j s}=\mathcal{E}_{n_{i r}^{<}}^{j s} \mathcal{E}_{X_{i r}}^{j s}+\Delta C_{i r}^{v,>}\left(\mathcal{E}_{\tilde{X}_{i r}}^{j s}\right), \\
& \Leftrightarrow \quad \mathcal{E}_{\tilde{n}_{i r}}^{j s}\left(\mathcal{E}_{\tilde{X}_{i r}}^{j s}\right)=\mathcal{E}_{n_{i r}^{<}}^{j s}+\frac{\Delta C_{i r}^{v,>}\left(\mathcal{E}_{\tilde{X}_{i r}}^{j s}\right)}{\mathcal{E}_{\tilde{X}_{i r}}^{j s}},
\end{aligned}
$$

where $\Delta C_{i r}^{v,>}$ denotes the cost increase in production extension introduced in Eq. (A.31). If $j s$ 's share remains unchanged ( $\mathcal{E}_{s_{i r \leftarrow j s} s}^{j s}=s_{i r \leftarrow j s}$ ), then $\mathcal{E}_{\bar{X}_{i r}}^{j s}=\mathcal{E}_{X_{i r}}$ (cf. Eq. (A.49)), and, according to assumption (ii), also $\mathcal{E}_{\bar{n}_{i r}}^{j s}=\mathcal{E}_{\bar{n}_{i r}}$ hold true. Inserting these into Eq. (A.52a) permits to derive $\mathcal{E}_{n_{i r}^{<}}^{j s}$ as

$$
\mathcal{E}_{n_{i r}^{<}}^{j s}=\mathcal{E}_{\bar{n}_{i r}}-\frac{\Delta C_{i r}^{v,>}\left(\mathcal{E}_{X_{i r}}\right)}{\mathcal{E}_{X_{i r}}} .
$$

Concluding, agent $j s$ 's estimate on $i r$ 's supply curve in the next timestep reads

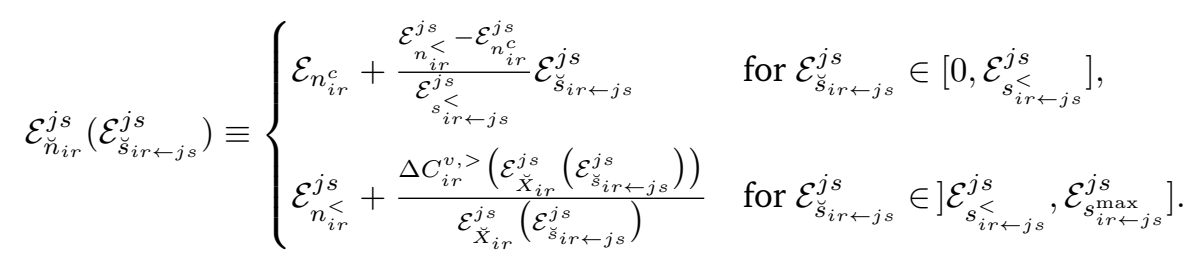

For the optimization procedure of the purchasing step (see Section 3.1.1), it is convenient to write $\mathcal{E}_{\tilde{n}_{i r}}^{j s}=$ $\mathcal{E}_{\breve{n}_{i r}}^{j s}\left(\breve{D}_{i r \leftarrow j s}\right)$ in terms of $\breve{D}_{i r \leftarrow j s}$ by concatenating Eq. (A.53) with the function defined in Eq. (A.47b), which yields

$$
\mathcal{E}_{\breve{n}_{i r}}^{j s}\left(\breve{D}_{i r \leftarrow j s}\right) \equiv\left(\mathcal{E}_{\breve{n}_{i r}}^{j s} \circ \mathcal{E}_{\breve{s}_{i r \leftarrow j s}^{j s}}^{j s}\right)\left(\breve{D}_{i r \leftarrow j s}\right) .
$$

Transport penalty. To render the baseline state of the economy stable with respect to idiosyncratic shocks, penalties in form of extra costs have to be assumed if one or more demands deviate from their baseline values. The corresponding penalty function for firm $j s$ and input commodity $i$ may be written as

$$
\mathcal{E}_{C_{i \rightarrow j s}^{\text {pen }}}\left(\left\{\breve{D}_{i r^{\prime} \leftarrow j s}\right\}_{r^{\prime}}\right) \equiv \sum_{r^{\prime}} \Delta \operatorname{TP}_{i r^{\prime} \rightarrow j s}\left(\breve{D}_{i r^{\prime} \leftarrow j s}\right),
$$

where $\mathcal{E}_{C_{i \rightarrow j s}^{\text {pen }}}$ is a function of the demand requests $\left\{\breve{D}_{i r^{\prime} \leftarrow j s}\right\}_{r^{\prime}}$ that $j s$ addresses to its suppliers $\left\{i r^{\prime}\right\}_{r^{\prime}}$ and $\Delta \mathrm{TP}_{i r \rightarrow j s}$ denotes the transport penalty to be discussed in the following.

We assume that the transportation costs in the baseline state are negligible compared to the value of the transported commodities. Extra costs only arise in non-equilibrium situations if agent $j s$ 's demand requests fluctuate, and means of transportation, e. g., vessels or trucks, cannot be used to their capacity. We assume that the transport penalties for each input commodity $i$ assume the form

$$
\Delta \operatorname{TP}_{i r \rightarrow j s}\left(\breve{D}_{i r \leftarrow j s}\right) \equiv \Delta n_{j}^{\text {tp }}\left(\frac{Z_{i r \rightarrow j s}^{*}-\breve{D}_{i r \leftarrow j s}}{Z_{i r \rightarrow j s}^{*}}\right)^{2}+\Delta n_{j}^{\text {tp }, \min }\left|Z_{i r \rightarrow j s}^{*}-\breve{D}_{i r \leftarrow j s}\right|
$$


where the coefficients $\Delta n_{j}^{\text {tp }}$ and $\Delta n_{j}^{\text {tp,min }}$ are allowed to vary among sectors. For large relative deviations, the quadratic term in Eq. (A.55) dominates and ensures the system to be stable, whereas the linear term in this equation guarantees the baseline equilibrium to be stable with respect to small deviations. For that, $\Delta n_{i}^{\text {tp, min }}$ has to be chosen sufficiently large as discussed in Appendix A.4.

Cost minimization. The expected purchasing costs of a firm $j s$ read

$$
\mathcal{E}_{C_{i \rightarrow j s}}\left(\left\{\breve{D}_{i r^{\prime} \leftarrow j s}\right\}_{r^{\prime}}\right) \equiv \underbrace{\sum_{r^{\prime}} \mathcal{E}_{\breve{n}_{i r^{\prime}}}^{j s}\left(\breve{D}_{i r^{\prime} \leftarrow j s}\right) \breve{D}_{i r^{\prime} \leftarrow j s}}_{\text {expected costs for purchases }}+\underbrace{\mathcal{E}_{C_{i \rightarrow j s}^{\text {pen }}}\left(\left\{\breve{D}_{i r^{\prime} \leftarrow j s}\right\}_{r^{\prime}}\right)}_{\begin{array}{c}
\text { expected additional costs } \\
\text { for transport }
\end{array}} .
$$

They are a function of the demand requests $\left\{\breve{D}_{i r^{\prime} \leftarrow j s}\right\}_{r^{\prime}}$ firm $j s$ addresses to its suppliers and depend upon the expected supply curves $\left\{\mathcal{E}_{\tilde{n}_{i r^{\prime}}}^{j s}\right\}_{r^{\prime}}$ of $j s^{\prime}$ s suppliers as well as upon transport costs $\mathcal{E}_{C_{i \rightarrow j s}^{\text {pen }} \text {. }}$.

A firm $j s$ decides on the optimal distribution of its demand requests among its suppliers by minimizing expected purchasing costs, separately for each commodity $i$, under the constraints that (i) its cumulative demand $D_{i \leftarrow j s}$ is met, and (ii) individual demand requests must not exceed the amounts $\left\{\mathcal{E}_{D_{i r^{\prime} \leftarrow j s}^{\max }}\right\}_{r^{\prime}}$ (see Eq. (A.50)) that $j s$ expects its suppliers to be able to deliver in the next timestep,

$$
\begin{aligned}
& \left\{D_{i r^{\prime} \leftarrow j s}\right\}_{r^{\prime}}=\underset{\left\{\breve{D}_{i r^{\prime} \leftarrow j s}\right\}_{r^{\prime}}}{\operatorname{argmin}} \mathcal{E}_{C_{i \rightarrow j s}}\left(\left\{\breve{D}_{i r^{\prime} \leftarrow j s}\right\}_{r^{\prime}}\right) \\
& \text { subject to } \sum_{r^{\prime}} \breve{D}_{i r^{\prime} \leftarrow j s}=D_{i \rightarrow j s} \text { and } 0 \leq \breve{D}_{i r \leftarrow j s} \leq \mathcal{E}_{D_{i r \leftarrow j s}^{\max }}^{j s} \forall r .
\end{aligned}
$$

The reservation price corresponding to a demanded quantity $D_{i r \leftarrow j s}$ is then given by

$$
n_{i r \leftarrow j s} \equiv \mathcal{E}_{\tilde{n}_{i r}}^{j s}\left(D_{i r \leftarrow j s}\right) .
$$

The purchase is done by sending each demand request $\left(D_{i r \leftarrow j s}, n_{i r \leftarrow j s}\right)$ to each supplier $i r$.

\section{Appendix A.3. Consumers}

Since commodities are perfect complements, consumer $j s$ has a separate consumption for each input commodity $i$, which may be written as

$$
\mathcal{C}_{i \rightarrow j s} \equiv \min \left[\mathcal{C}_{i \rightarrow j s}^{*} \cdot\left(\frac{\bar{n}_{i \rightarrow j s}^{l}}{\bar{n}_{i \rightarrow j s}^{*}}\right)^{\varepsilon_{i \rightarrow j s}^{c}}, \hat{U}_{i \rightarrow j s}\right] .
$$

It varies isoelastically with the corresponding consumer price $\bar{n}_{i \rightarrow j s}^{l}$ (see Eq. (A.13)) for commodity $i$. Further, in the above equation, $\mathcal{C}_{i \rightarrow j s}^{*}, \varepsilon_{i \rightarrow j s}^{c} \in\left[-1,0\left[\right.\right.$, and $n_{i \rightarrow j s}^{*}$ denote baseline consumption, consumption price elasticity, and the normalized consumer price in the baseline state, respectively. Consumption price elasticities may differ among input commodities, which permits to distinguish consumption from investment commodities, and due to price normalization, we have $n_{i \rightarrow j s}^{*}=1$ according to Eq. (A.13). The minimum condition in Eq. (A.59) reflects that consumption may be limited by a reduced availability $\hat{U}_{i \rightarrow j s}$ of commodity $i$ (see Eq. (A.14)) if supply shortages arise in the disaster aftermath.

For consumers, besides consumption, which is done in parallel with the production step of firms only the purchasing step, is relevant, where they decide upon their demand and its distribution. Having 'naive expectations', regional consumers' assume that their consumer prices for input commodities remain unchanged in the next timestep. For that, they calculate their demand for input commodity $i$ by assuming that they will consume (use) the amount $\mathcal{E}_{U_{i \rightarrow j s}} \equiv \mathcal{C}_{i \rightarrow j s}^{*} \cdot\left(\frac{\bar{n}_{i \rightarrow j s}^{l}}{\bar{n}_{i \rightarrow j s}^{*}}\right)^{\varepsilon_{i \rightarrow j s}^{c}}$ in the next timestep. For each input commodity, they may then calculate their demand as well as the optimal demand distribution from Eqs. (A.44) and (A.57), respectively. 
Appendix A.4. First-order condition for locally stable baseline equilibrium

Since the economy is demand-driven, the baseline equilibrium is locally stable if for each agent $j s$ and each input commodity $i$, the baseline demand distribution $\left\{D_{i r^{\prime} \leftarrow j s}^{*}\right\}_{r^{\prime}}$ minimizes expected purchasing costs with respect to all perturbations of this baseline state keeping cumulative demand $D_{i \leftarrow j s}^{*}=\sum_{r^{\prime}} D_{i r^{\prime} \leftarrow j s}^{*}$ unchanged. In the following, we restrict ourselves to a firm $j s$ that has only two suppliers of commodity $i$ to which it addresses the demands $D_{1}$ and $D_{2}$. We then have to ensure that $D_{1}^{*}$ and $D_{2}^{*}$ are the solutions of the following constraint optimization problem

$$
\underset{\left\{\breve{D}_{1}, \breve{D}_{2}\right\}}{\operatorname{argmin}}\left[\mathcal{E}_{C_{i \rightarrow j s}}\right] \quad \text { subject to } \quad \breve{D}_{1}+\breve{D}_{2}=D_{i \leftarrow j s}^{*} .
$$

Taking into account that the constraint in the above equations permits to write $D_{2}$ in terms of $D_{1}$, the first-order condition may be written as

$$
\begin{aligned}
0 \leq & \left.\frac{\partial^{+} \mathcal{E}_{C_{i \rightarrow j s}}}{\partial D_{1}}\right|_{*} \\
\stackrel{\text { (A.55),(A.56) }}{\Leftrightarrow} \quad & \left.\frac{\partial^{+} \mathcal{E}_{n_{i 1}}^{j s}}{\partial D_{1}}\right|_{*} D_{1}^{*}+\left.\mathcal{E}_{\tilde{n}_{i 1}}^{j s}\right|_{*}+\left.\frac{\partial^{+} \mathcal{E}_{C_{i \rightarrow j s}^{\text {pen }}}}{\partial D_{1}}\right|_{*} \\
& +\left.\frac{\partial D_{2}}{\partial D_{1}}\right|_{*}\left[\left.\frac{\partial^{-} \mathcal{E}_{\tilde{n}_{i 2}}^{j s}}{\partial D_{2}}\right|_{*} D_{2}^{*}+\left.\mathcal{E}_{\tilde{n}_{i 2}}^{j s}\right|_{*}+\left.\frac{\partial^{-} \mathcal{E}_{C_{i \rightarrow j s}^{\text {pen }}}}{\partial D_{2}}\right|_{*}\right] \\
\text { (A.25),(A.29),(A.54),(A.53) } \quad 0 \leq & 0+1+\Delta n_{j s}^{\text {tp,min }}-\left[\pi_{i 2}^{*}+1-\Delta n_{j s}^{\text {tp,min }}\right] \\
\Leftrightarrow \quad \Delta n_{j s}^{\text {tp,min }} \geq & \frac{\pi_{i 2}}{2} .
\end{aligned}
$$

Here, $\partial^{+}(\cdot) / \partial D$ and $\partial^{-}(\cdot) / \partial D$ denote right-hand side and left-hand side partial derivatives, respectively. And $\left.\right|_{*}$ denotes that variables are evaluated and derivatives are taken at the baseline state. We see from Eq. (A.60) that the first-order condition can be fulfilled by choosing $\Delta n_{j s}^{\text {tp, min }} \geq \pi_{i}^{*} / 2 \geq \pi_{i r}^{*} / 2 \forall r$, where, in the last equality, we have taken into account that, according to Eq. (A.27), the exogenously set sectoral monopolistic markup $\pi_{i}^{*}$ may be larger than the one of the individual supplier $\pi_{i r}^{*}$. 


\section{Appendix B. Tables}

Table B.1: Parameters of acclimate; values used in the numerical simulations unless stated otherwise.

\begin{tabular}{llllll}
\hline Variable & Description & Unit & Scope & Eq. & Value \\
\hline$\Delta t$ & timestep & time & global & & 1 day \\
$\omega_{i}$ & upper storage limit & - & sector & (A.11) & 3 \\
$\Psi_{i}$ & storage fill factor & time & sector & (A.10) & 15 days \\
$\beta_{i}$ & prod. extension factor & - & sector & (A.15) & 1.1 \\
$\pi_{i}^{*}$ & baseline monopolistic markup & price & sector & (A.27) & 0.05 \\
$\Delta n_{i}^{\text {in }, v,>}$ & unit extra variable prod. costs in prod. extension & price & sector & (A.22) & 5 \\
$\Delta n_{i}^{\text {tp }}$ & coefficient of quadratic transport penalty & value & sector & (A.55) & $0.08 \mathrm{USD}^{-10}$ \\
$\Delta n_{i}^{\text {tp,min }}$ & coefficient of linear transport penalty & price & sector & (A.55) & $0.025 \mathrm{USD}^{-1}$ \\
$\tau_{i \rightarrow j s}$ & storage balance time scale & time & storage & (A.44), (5) & 2 days \\
$\varepsilon_{i \rightarrow j s}^{c}$ & consumption price elasticity & - & storage (consumer) & (A.59), (8) & -0.5 \\
$\lambda_{j s}^{(t)}$ & production forcing & & firm & (A.15) & 0.001 \\
\hline
\end{tabular}


Table B.2: Exogenous variables of acclimate; values derived from MRIO-tables.

\begin{tabular}{lllll}
\hline Variable & Description & Unit & Scope & Eq. \\
\hline$Z_{i r \rightarrow j s}^{*}$ & baseline flow & $\frac{\text { quantity }}{\text { time }}$ & connection & (A.1) \\
$D_{i r \leftarrow j s}^{*}$ & baseline demand request & $\frac{\text { quantity }}{\text { time }}$ & connection & (A.5) \\
$I_{i \rightarrow j s}^{*}$ & baseline input flow & $\frac{\text { quantity }}{\text { time }}$ & storage & (A.9) \\
$U_{i \rightarrow j s}^{*}$ & baseline use & $\frac{\text { quantity }}{\text { time }}$ & storage & (A.9) \\
$S_{i \rightarrow j s}^{*}$ & baseline storage content & quantity & storage & (A.10) \\
$S_{i \rightarrow j s}^{\text {max }}$ & maximum storage content & quantity & storage & (A.11) \\
$X_{j s}^{*}$ & baseline production level & $\frac{\text { quantity }}{\text { time }}$ & firm & (A.2) \\
$a_{i \rightarrow j s}$ & technology coefficient & - & firm & (A.16) \\
$n_{j s}^{v, *}$ & baseline unit variable production costs & price & firm & (A.26) \\
$\pi_{j s}^{*}$ & baseline monopolistic markup & price & firm & (A.27) \\
VA $_{j s}^{*}$ & baseline value added & $\frac{\text { value }}{\text { time }}$ & firm & (A.25) \\
$\mathcal{C}_{i \rightarrow j s}^{*}$ & baseline consumption & $\frac{\text { quantity }}{\text { time }}$ & consumer & (A.3) \\
\hline
\end{tabular}

Table B.3: Endogenous variables of acclimate.

\begin{tabular}{lllll}
\hline Variable & Description & Unit & Scope & Eq. \\
\hline$l^{D, T, I,(t)}$ & direct/total/indirect daily losses & $\frac{\text { quantity }}{\text { time }}$ & global & (9), (10), (11) \\
$L^{D, T, I,(t)}$ & direct/total/indirect cumul. losses & quantity & global & (12) \\
$D_{i r \leftarrow j s}^{(t)}$ & demand request & $\frac{\text { quantity }}{\text { time }}$ & connection & (A.57), (7) \\
$n_{i r \leftarrow j s}^{(t)}$ & reservation price & price & connection & (A.58) \\
$Z_{i r \leftarrow j s}^{(t)}$ & supply flow & $\frac{\text { quantity }}{\text { time }}$ & connection & (A.37) \\
$T_{i r \rightarrow j s}^{(t)}$ & transport stock & quantity & connection & (A.6) \\
$I_{i \rightarrow j s}^{(t)}$ & input flow & $\frac{\text { quantity }}{\text { time }}$ & storage & (A.8) \\
$S_{i \rightarrow j s}^{(t)}$ & storage content & quantity & storage & (A.12) \\
$\hat{U}_{i \rightarrow j s}^{(t)}$ & possible use from storage & $\frac{\text { quantity }}{\text { time }}$ & storage & (A.14) \\
$U_{i \rightarrow j s}^{(t)}$ & use from storage & $\frac{\text { quantity }}{\text { time }}$ & storage & (A.36) \\
$\bar{n}_{i \rightarrow j s}^{l,(t)}$ & unit commodity costs & price & agent & (A.13) \\
$T_{i \rightarrow j s}^{\text {def, }(t)}$ & transport deficit & $\frac{\text { quantity }}{\text { time }}$ & agent & (A.46) \\
$\Delta \operatorname{TP}_{i r \rightarrow j s}^{(t)}$ & transport penalties & $\frac{\text { value }}{\text { time }}$ & agent & (A.55)
\end{tabular}




\begin{tabular}{|c|c|c|c|c|}
\hline Variable & Description & Unit & Scope & Eq. \\
\hline$\Delta n_{j}^{\mathrm{tp}, \min }$ & linear coeff. of trans. penalty & $\frac{\text { value }}{\text { time }}$ & agent & (A.55) \\
\hline$\Delta S_{i \rightarrow j s}^{(t)}$ & storage shortage & $\frac{\text { value }}{\text { time }}$ & agent & (A.45) \\
\hline $\mathcal{E}_{C_{i \rightarrow j s}^{(t)}}^{\text {pen }}$ & transport penalties & $\frac{\text { value }}{\text { time }}$ & agent & (A.54) \\
\hline $\mathcal{E}_{\tilde{n}_{i r}}^{j s,(t)}$ & expected supply curve & price & agent & (A.53) \\
\hline$D_{i \leftarrow j s}$ & total demand & $\frac{\text { quantity }}{\text { time }}$ & agent & (A.44), (5) \\
\hline $\mathcal{E}_{C_{i \rightarrow j s}}$ & expected purchasing costs & $\frac{\text { value }}{\text { time }}$ & agent & (A.56), (6) \\
\hline $\bar{n}_{j s}^{p,(t)}$ & average reservation price & price & firm & (A.39) \\
\hline$D_{j s \leftarrow}^{(t)}$ & incoming demand & $\frac{\text { quantity }}{\text { time }}$ & firm & (A.20), (1) \\
\hline $\bar{n}_{j s}^{(t)}$ & selling price & price & firm & (A.38) \\
\hline$R_{j s}^{(t)}$ & revenue & $\frac{\text { value }}{\text { time }}$ & firm & (A.18) \\
\hline$n_{j s}^{c,(t)}$ & unit production costs & price & firm & (A.29) \\
\hline$C_{j s}^{l,(t)}$ & costs for commodity inputs & $\frac{\text { value }}{\text { time }}$ & firm & (A.21) \\
\hline$n_{j s}^{v,(t)}$ & unit variable production costs & price & firm & (A.26) \\
\hline$C_{j s}^{v,(t)}$ & variable production costs & $\frac{\text { value }}{\text { time }}$ & firm & (A.22) \\
\hline$C_{j s}^{(t)}$ & total costs & $\frac{\text { value }}{\text { time }}$ & firm & (A.28), (2) \\
\hline$\Delta C_{j s}^{v,>,(t)}$ & extra variable production costs in prod. extension & $\frac{\text { value }}{\text { time }}$ & firm & (A.31) \\
\hline$\Pi_{j s}^{(t)}$ & profit & $\frac{\text { value }}{\text { time }}$ & firm & (A.32), (4) \\
\hline $\mathrm{VA}_{j s}^{(t)}$ & value added & $\frac{\text { value }}{\text { time }}$ & firm & (A.23) \\
\hline$X_{j s}^{\mathrm{opt},(t)}$ & optimal production level & $\frac{\text { quantity }}{\text { time }}$ & firm & (A.34) \\
\hline$\hat{X}_{j s}^{(t)}$ & productive capacity & $\frac{\text { quantity }}{\text { time }}$ & firm & (A.15) \\
\hline$X_{j s}^{(t)}$ & production level & $\frac{\text { quantity }}{\text { time }}$ & firm & (A.35), (3) \\
\hline $\mathcal{E}_{\bar{n}_{j s}}^{(t)}$ & offer price & price & firm & (A.40) \\
\hline $\mathcal{E}_{R_{j s}}^{(t)}$ & expected revenue & price & firm & (A.41) \\
\hline $\mathcal{E}_{\Pi_{j s}}^{(t)}$ & expected profit & $\frac{\text { value }}{\text { time }}$ & firm & (A.41) \\
\hline $\mathcal{E}_{\hat{X}_{j s}}^{(t)}$ & expected productive capacity & $\frac{\text { quantity }}{\text { time }}$ & firm & (A.42) \\
\hline $\mathcal{E}_{X_{j s}}^{(t)}$ & expected optimal production level & $\frac{\text { quantity }}{\text { time }}$ & firm & (A.43) \\
\hline $\mathcal{C}_{i \rightarrow j s}^{(t)}$ & consumption & $\frac{\text { quantity }}{\text { time }}$ & consumer & (A.59), (8) \\
\hline
\end{tabular}


Table B.4: Countries used in the numerical simulations.

\begin{tabular}{|c|c|}
\hline ISO3-Code & Country Name \\
\hline AGO & Angola \\
\hline BEN & Benin \\
\hline BWA & Botswana \\
\hline BFA & Burkina Faso \\
\hline BDI & Burundi \\
\hline CMR & Cameroon \\
\hline CPV & Cap Verde \\
\hline CAF & Central African Republic \\
\hline TCD & Chad \\
\hline CIV & Côte d'Ivoire \\
\hline ERI & Eritrea \\
\hline GAB & Gabon \\
\hline GMB & Gambia \\
\hline GHA & Ghana \\
\hline GIN & Guinea \\
\hline KEN & Kenya \\
\hline LSO & Lesotho \\
\hline LBR & Liberia \\
\hline MDG & Madagascar \\
\hline MWI & Malawi \\
\hline MLI & Mali \\
\hline MRT & Mauritania \\
\hline MUS & Mauritius \\
\hline MOZ & Mozambique \\
\hline NAM & Namibia \\
\hline NER & Niger \\
\hline NGA & Nigeria \\
\hline COG & Republic of the Congo \\
\hline
\end{tabular}

\begin{tabular}{|c|c|}
\hline ISO3-Code & Country Name \\
\hline RWA & Rwanda \\
\hline SEN & Senegal \\
\hline SYC & Seychelles \\
\hline SLE & Sierra Leone \\
\hline SOM & Somalia \\
\hline ZAF & South Africa \\
\hline LKA & Sri Lanka \\
\hline SUR & Suriname \\
\hline SWZ & Swaziland \\
\hline TGO & Togo \\
\hline UGA & Uganda \\
\hline ZMB & Zambia \\
\hline ZWE & Zimbabwe \\
\hline $\mathrm{CHN}$ & China \\
\hline MNG & Mongolia \\
\hline VNM & Vietnam \\
\hline AUT & Austria \\
\hline BEL & Belgium \\
\hline BGR & Bulgaria \\
\hline HRV & Croatia \\
\hline CYP & Cyprus \\
\hline CZE & Czech Republic \\
\hline DNK & Denmark \\
\hline EST & Estonia \\
\hline FIN & Finland \\
\hline FRA & France \\
\hline DEU & Germany \\
\hline GRC & Greece \\
\hline HUN & Hungary \\
\hline IRL & Ireland \\
\hline
\end{tabular}




\begin{tabular}{|c|c|}
\hline ISO3-Code & Country Name \\
\hline ITA & Italy \\
\hline LVA & Latvia \\
\hline LTU & Lithuania \\
\hline LUX & Luxembourg \\
\hline MLT & Malta \\
\hline NLD & Netherlands \\
\hline POL & Poland \\
\hline PRT & Portugal \\
\hline ROU & Romania \\
\hline SVK & Slovakia \\
\hline SVN & Slovenia \\
\hline ESP & Spain \\
\hline SWE & Sweden \\
\hline GBR & United Kingdom \\
\hline ARM & Armenia \\
\hline AZE & Azerbaijan \\
\hline BLR & Belarus \\
\hline EST & Estonia \\
\hline GEO & Georgia \\
\hline KAZ & Kazakhstan \\
\hline KGZ & Kyrgyzstan \\
\hline LVA & Latvia \\
\hline LTU & Lithuania \\
\hline RUS & Russia \\
\hline TJK & Tajikistan \\
\hline TKM & Turkmenistan \\
\hline UKR & Ukraine \\
\hline UZB & Uzbekistan \\
\hline ARG & Argentina \\
\hline BOL & Bolivia \\
\hline
\end{tabular}

\begin{tabular}{|c|c|}
\hline ISO3-Code & Country Name \\
\hline BRA & Brazil \\
\hline CHL & Chile \\
\hline COL & Colombia \\
\hline ECU & Ecuador \\
\hline GUY & Guyana \\
\hline PRY & Paraguay \\
\hline PER & Peru \\
\hline SUR & Suriname \\
\hline URY & Uruguay \\
\hline VEN & Venezuela \\
\hline DZA & Algeria \\
\hline BHR & Bahrain \\
\hline CYP & Cyprus \\
\hline DJI & Djibouti \\
\hline EGY & Egypt \\
\hline IRN & Iran \\
\hline IRQ & Iraq \\
\hline ISR & Israel \\
\hline JOR & Jordan \\
\hline KWT & Kuwait \\
\hline LBN & Lebanon \\
\hline LBY & Libya \\
\hline MRT & Mauritania \\
\hline MAR & Morocco \\
\hline OMN & Oman \\
\hline PSE & Palestine \\
\hline QAT & Qatar \\
\hline WSM & Samoa \\
\hline SAU & Saudi Arabia \\
\hline SYR & Syria \\
\hline
\end{tabular}




\begin{tabular}{|c|c|}
\hline ISO3-Code & Country Name \\
\hline TUN & Tunisia \\
\hline TUR & Turkey \\
\hline ARE & United Arab Emirates \\
\hline YEM & Yemen \\
\hline ATG & Antigua and Barbuda \\
\hline ABW & Aruba \\
\hline BHS & Bahamas \\
\hline BRB & Barbados \\
\hline BLZ & Belize \\
\hline BMU & Bermuda \\
\hline VGB & British Virgin Islands \\
\hline CAN & Canada \\
\hline CYM & Cayman Islands \\
\hline CRI & Costa Rica \\
\hline CUB & Cuba \\
\hline DOM & Dominican Republic \\
\hline SLV & El Salvador \\
\hline GRL & Greenland \\
\hline GTM & Guatemala \\
\hline HTI & Haiti \\
\hline HND & Honduras \\
\hline JAM & Jamaica \\
\hline MEX & Mexico \\
\hline ANT & Netherlands Antilles \\
\hline NIC & Nicaragua \\
\hline PAN & Panama \\
\hline TTO & Trinidad and Tobago \\
\hline USA & United States of America \\
\hline AUS & Australia \\
\hline JPN & Japan \\
\hline
\end{tabular}

\begin{tabular}{ll}
\hline ISO3-Code & Country Name \\
\hline NZL & New Zealand \\
KOR & South Korea \\
\hline BRN & Brunei \\
KHM & Cambodia \\
IDN & Indonesia \\
LAO & Laos \\
MYS & Malaysia \\
MDV & Maldives \\
NPL & Nepal \\
PNG & Papua New Guinea \\
PHL & Philippines \\
LKA & Sri Lanka \\
THA & Thailand \\
\hline AFG & Afghanistan \\
BGD & Bangladesh \\
BTN & Bhutan \\
IND & India \\
\hline
\end{tabular}


Table B.5: Sectors used in the numerical simulations.

\begin{tabular}{ll}
\hline Code & Name \\
\hline AGRI & Agriculture \\
FISH & Fishing \\
MINQ & Mining and Quarrying \\
FOOD & Food \& Beverages \\
TEXL & Textiles and Wearing Apparel \\
WOOD & Wood and Paper \\
OILC & Petroleum, Chemical and Non-Metallic Mineral Products \\
METL & Metal Products \\
MACH & Electrical and Machinery \\
TREQ & Transport Equipment \\
MANU & Other Manufacturing \\
RECY & Recycling \\
ELWA & Electricity, Gas and Water \\
CONS & Construction \\
REPA & Maintenance and Repair \\
WHOT & Wholesale Trade \\
RETT & Retail Trade \\
GAST & Hotels and Restaurants \\
TRAN & Transport \\
COMM & Post and Telecommunications \\
FINC & Financial Intermediation and Business Activities \\
ADMI & Public Administration \\
EDHE & Education, Health and Other Services \\
HOUS & Private Households \\
OTHE & Others \\
REXI & Re-export \& Re-import \\
FCON & Final consumption \\
& \\
\hline &
\end{tabular}

